\title{
3D Boranil Complexes with Aggregation-amplified Circularly Polarized Luminescence
}

Kang Li, Honghan $\mathrm{Ji}^{\dagger}{ }^{\dagger}$ Zeren Yang, ${ }^{\dagger}$ Wenzeng Duan, ${ }^{*}{ }^{\dagger}$ Yudao Ma, ${ }^{\dagger}$ Houting Liu, ${ }^{\dagger}$ Huaiwei Wang, Shuwen Gong* ${ }^{\dagger} \dagger$

${ }^{\dagger}$ Institute of Functional Organic Molecules and Materials, School of Chemistry and Chemical Engineering, Liaocheng University, No.1 Hunan Road, Liaocheng, 252000, People's Republic of China.

\$Department of Chemistry, Shandong University, Shanda South Road No.27, Jinan, 250100, People's Republic of China.

*E-mail: duanwenzeng@lcu.edu.cn.

*E-mail: gongshw@lcu.edu.cn.

\section{Table of content}

1. Experimental details S2

2. Photophysical properties $\mathrm{S} 2-\mathrm{S} 23$

3. General Procedure for crystal preparation and measurement $\mathrm{S} 23-\mathrm{S} 28$

4. Copies of NMR and HRMS Spectra S29-S43

5. Coordinates of optimized structures S43-S53

6. References S53 


\section{Experimental details}

\subsection{Theoretical calculations}

All the calculations were based on density functional theory (DFT) with B3LYP functional and 6-31G(d) basis set. Solvent (dichloromethane) was considered in all the calculations. The UV-vis absorptions of the compounds (vertical excitation) were calculated with the TD-DFT method based on the optimized ground state geometry $\left(\mathrm{S}_{0}\right.$ state). For the fluorescence emission spectra, the emission wavelength was calculated based on the optimized excited states ( $\mathrm{S}_{1}$ state). All these calculations were performed with Gaussian 09 software. ${ }^{1}$

\subsection{Chiral HPLC analysis}

Chiral HPLC analysis of $\left(R_{p}\right) /\left(S_{p}\right)$-3a-3e was performed using the Shimadzu LC-20AD HPLC workstation with mixed solvents of hexane and ethanol with the ratio of 7: 3 as an eluent.

HPLC analysis conditions:

Column: CHIRALPAK AD $4.6 \mathrm{~mm} \times 250 \mathrm{~mm}$

Injection: $15.0 \mu \mathrm{L}$

Flow rate: $1.0 \mathrm{~mL} \cdot \mathrm{min}^{-1}$

Abs. detector: UV $410 \mathrm{~nm}$

Temperature: $30^{\circ} \mathrm{C}$.

\section{Photophysical properties}

\subsection{UV-vis absorption spectra}

UV/vis absorption spectra were measured at room temperature on a Shimadzu UV-1750 spectrophotometer with a resolution of $1.0 \mathrm{~nm}$, using quartz cuvettes of $1 \mathrm{~cm}$ path length.

\subsection{Emission spectra}

Emission spectra were recorded on a FLUOROMAX-4 spectrometer under air atmosphere at room temperature under the following conditions: excitation wavelength $=450 \mathrm{~nm}$, an excitation-slit width of $2 \mathrm{~nm}$; an emission-slit width of $2 \mathrm{~nm}$.

\subsection{CD and CPL spectra}

Circular dichroism (CD) spectra of all complexes in DCM solution $\left(5.0 \times 10^{-5} \mathrm{~mol} \cdot \mathrm{L}^{-1}\right)$ were measured using a JASCO J-820 spectropolarimeter, at room temperature (cell length: $10 \mathrm{~mm}$, bandwidth: $1 \mathrm{~nm}$, scanning speed: $100 \mathrm{~nm} / \mathrm{min}$, data pitch: $1 \mathrm{~nm}$, accumulations: 2). The $C D$ spectra were approximated using the simple moving average (SMA) method. Circularly polarized luminescence (CPL) spectra in DCM solution $\left(5.0 \times 10^{-5} \mathrm{~mol} \cdot \mathrm{L}^{-1}\right)$ for $\left(R_{p}\right) /\left(S_{p}\right)-\mathbf{3 a}-\mathbf{3 b}$ and $\left(R_{p}\right) /\left(S_{p}\right)$-3d-3e were recorded with a JASCO CPL-300 spectrofluoropolarimeter at room temperature. The CPL spectra were approximated using the SMA method. $\left(\lambda_{\mathrm{ex}}=400 \mathrm{~nm}\right.$, cell length: $10 \mathrm{~mm}$, Ex \& Em slit width: $3000 \mu \mathrm{m}$, scanning speed: $100 \mathrm{~nm} / \mathrm{min}$, data pitch: $1 \mathrm{~nm}$, accumulations: 8 ). 

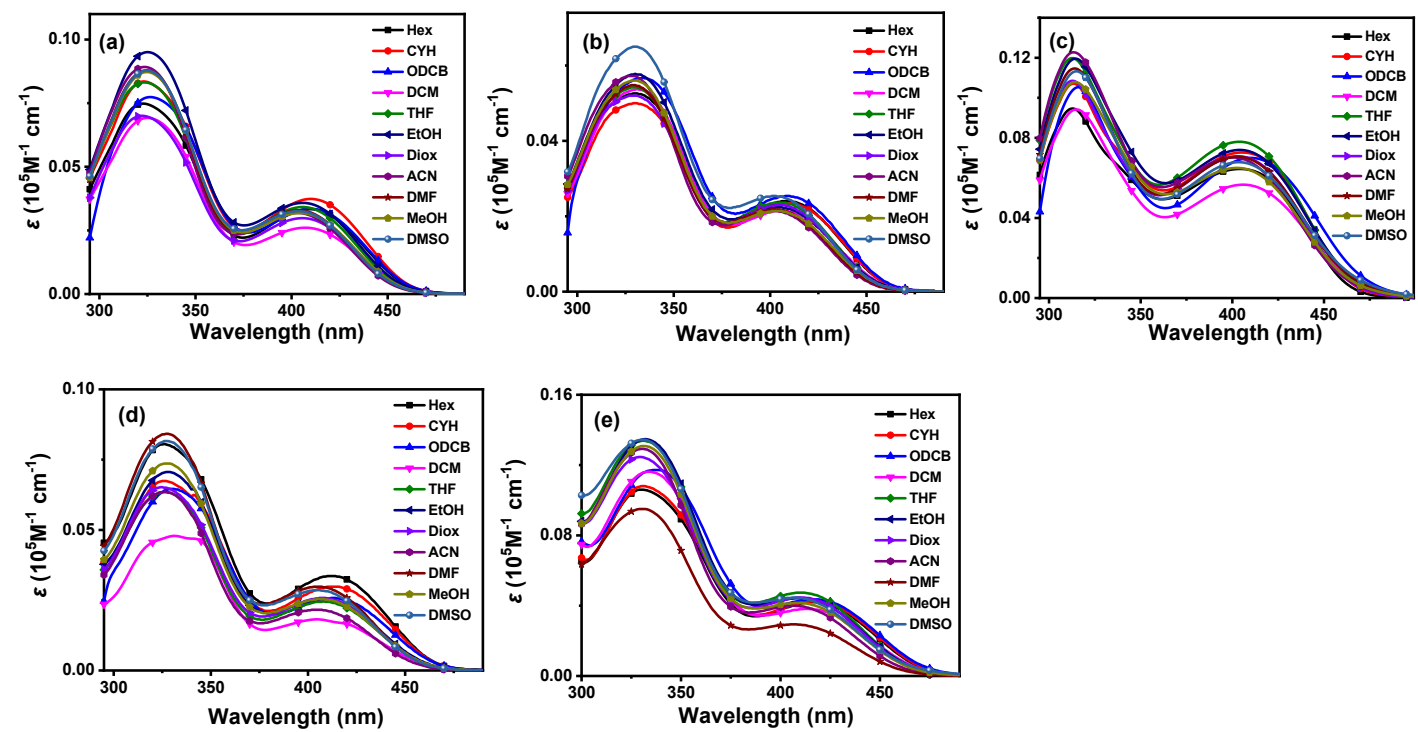

Figure S1. Absorption spectra of compounds $\left(R_{p}\right) /\left(S_{p}\right)-\mathbf{3 a}(\mathrm{a}),\left(R_{p}\right) /\left(S_{p}\right)-\mathbf{3 b}(\mathrm{b}),\left(R_{p}\right) /\left(S_{p}\right)-\mathbf{3 c}(\mathrm{c}),\left(R_{p}\right) /\left(S_{p}\right)-\mathbf{3 d}(\mathrm{d})$ and $\left(R_{p}\right) /\left(S_{p}\right)-3 \mathrm{e}(\mathrm{e})$ in various solvents $\left(5.0 \times 10^{-5} \mathrm{~mol} \cdot \mathrm{L}^{-1}\right)$ such as hexane $(\mathrm{Hex})$, cyclohexane $(\mathrm{CYH})$, 1,2-dichlorobenzene (ODCB), dichloromethane (DCM), tetrahydrofuran (THF), ethanol (EtOH), 1,4-dioxane (Diox), acetonitrile (ACN), N,N-dimethylformamide (DMF), methanol (MeOH), dimethyl sulfoxide (DMSO), respectively.
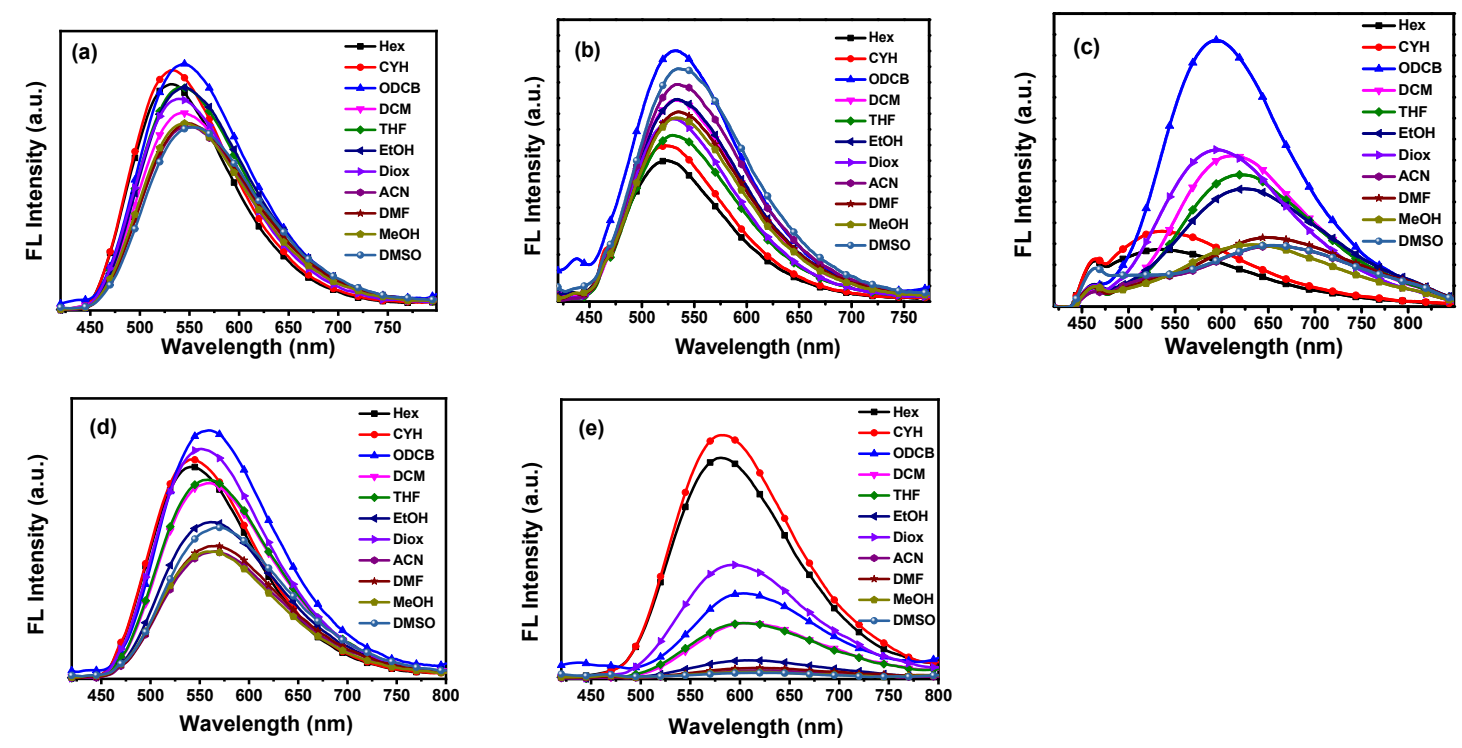

Figure S2. Emission spectra of compounds $\left(R_{p}\right) /\left(S_{p}\right)-\mathbf{3 a}(\mathrm{a}),\left(R_{p}\right) /\left(S_{p}\right)-\mathbf{3 b}(\mathrm{b}),\left(R_{p}\right) /\left(S_{p}\right)-\mathbf{3 c}(\mathrm{c}),\left(R_{p}\right) /\left(S_{p}\right)-\mathbf{3 d}(\mathrm{d})$ and $\left(R_{p}\right) /\left(S_{p}\right)$-3e (e) in various solvents $\left(5.0 \times 10^{-5} \mathrm{~mol} \cdot \mathrm{L}^{-1}\right)$ such as hexane (Hex), cyclohexane $(\mathrm{CYH})$, 1,2-dichlorobenzene (ODCB), dichloromethane (DCM), tetrahydrofuran (THF), ethanol (EtOH), 1,4-dioxane (Diox), acetonitrile (ACN), N,N-dimethylformamide (DMF), methanol (MeOH), dimethyl sulfoxide (DMSO), respectively. 

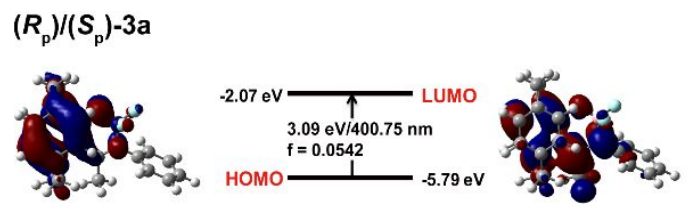

$\left(R_{\mathrm{p}}\right) /\left(S_{\mathrm{p}}\right)-3 \mathrm{C}$

$\left(R_{\mathrm{p}}\right) /\left(S_{\mathrm{p}}\right)-3 \mathrm{~d}$
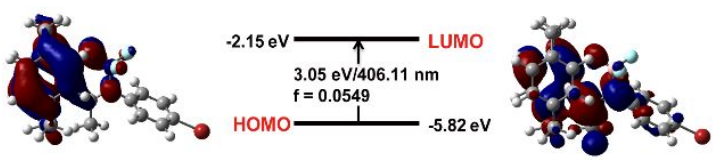

$\left(R_{\mathrm{p}}\right) /\left(S_{\mathrm{p}}\right)-3 \mathrm{e}$

Figure S3. Molecular orbital amplitude plots and energy levels of HOMOs and LUMOs of $\left(R_{p}\right) /\left(S_{p}\right)-3 \mathbf{a}$, $\left(R_{p}\right) /\left(S_{p}\right)-\mathbf{3 c},\left(R_{p}\right) /\left(S_{p}\right)-3 \mathbf{d}$ and $\left(R_{p}\right) /\left(S_{p}\right)-3 \mathbf{e}$ calculated at the theory level of B3LYP/ 6-31G(d,p).
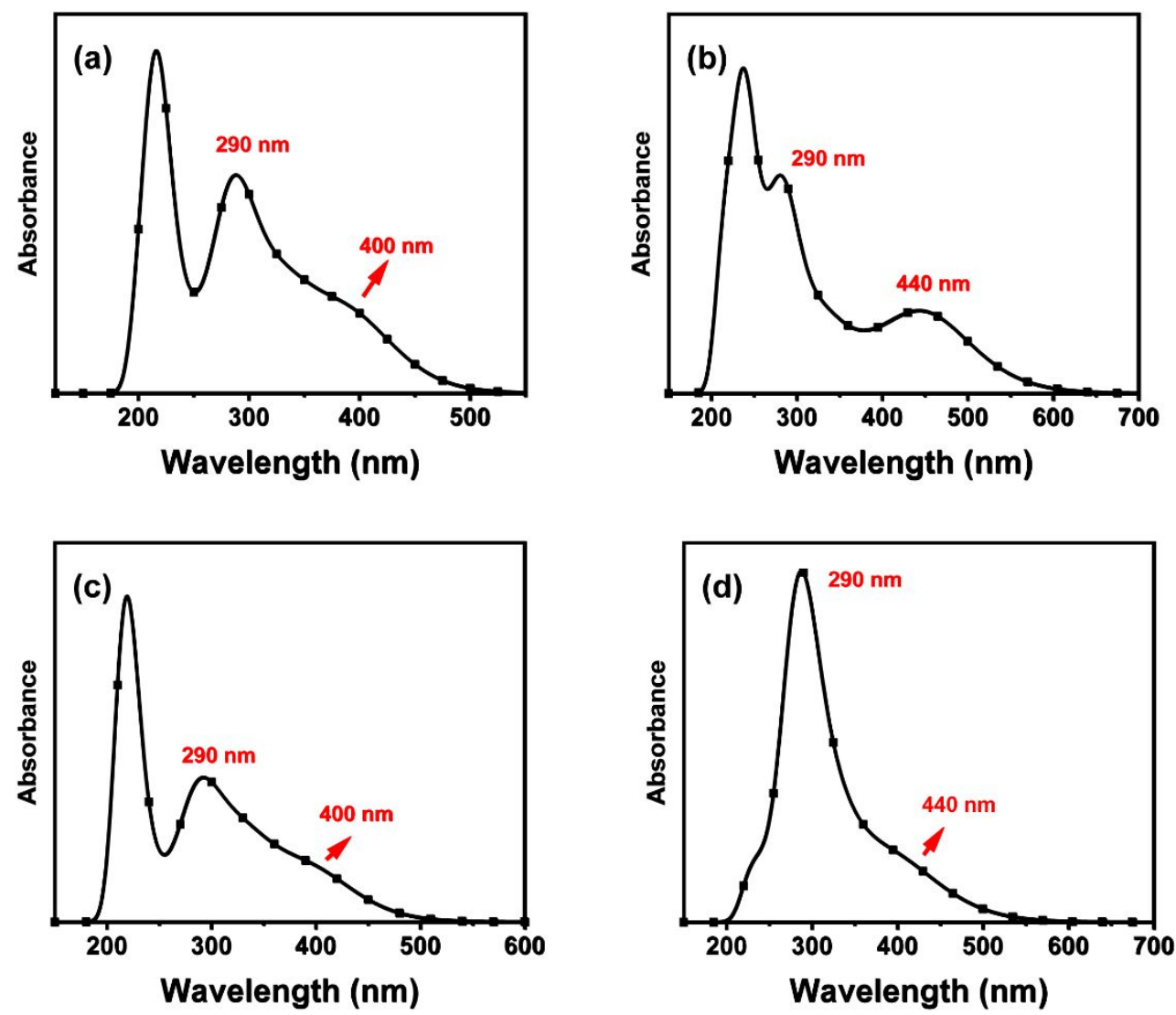

Figure S4. Calculated absorption spectra of compounds $\left(R_{p}\right) /\left(S_{p}\right)-\mathbf{3 a}(\mathrm{a}),\left(R_{p}\right) /\left(S_{p}\right)-\mathbf{3 c}(\mathrm{b}),\left(R_{p}\right) /\left(S_{p}\right)-\mathbf{3 d}(\mathrm{c})$ and $\left(R_{p}\right) /\left(S_{p}\right)-3 \mathbf{e}(\mathrm{d})$. 


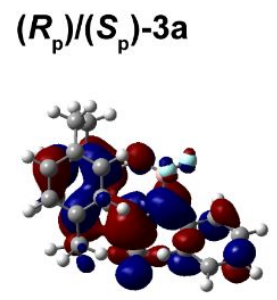

LUMO -2.57eV

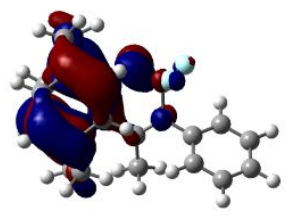

HOMO -5.45 eV
$\left(R_{\mathrm{p}}\right) /\left(S_{\mathrm{p}}\right)-3 \mathrm{c}$

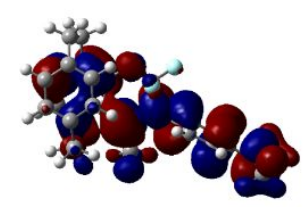

LUMO -2.44 eV

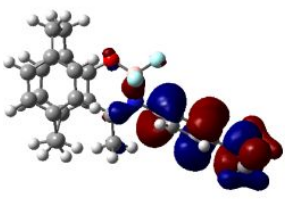

HOMO -4.99 eV
$\left(R_{\mathrm{p}}\right) /\left(S_{\mathrm{p}}\right)-3 \mathrm{~d}$

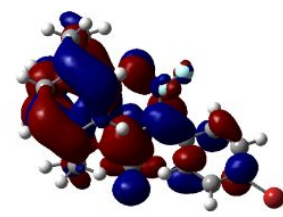

LUMO -2.64 eV

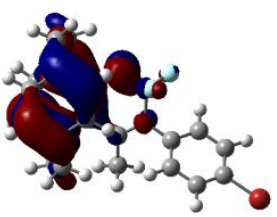

HOMO -5.49 eV
$\left(R_{p}\right) /\left(S_{p}\right)-3 e$

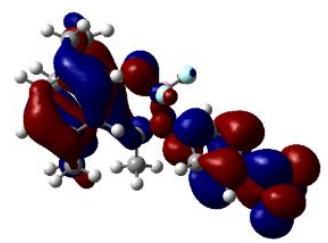

LUMO $-3.28 \mathrm{eV}$

Figure S5. Calculated frontier molecular orbitals for $\left(R_{p}\right) /\left(S_{p}\right)-\mathbf{3 a},\left(R_{p}\right) /\left(S_{p}\right)-\mathbf{3} \mathbf{c},\left(R_{p}\right) /\left(S_{p}\right)-\mathbf{3 d}$ and $\left(R_{p}\right) /\left(S_{p}\right)-\mathbf{3 e}$ and their orbital energies in the optimized excited state.

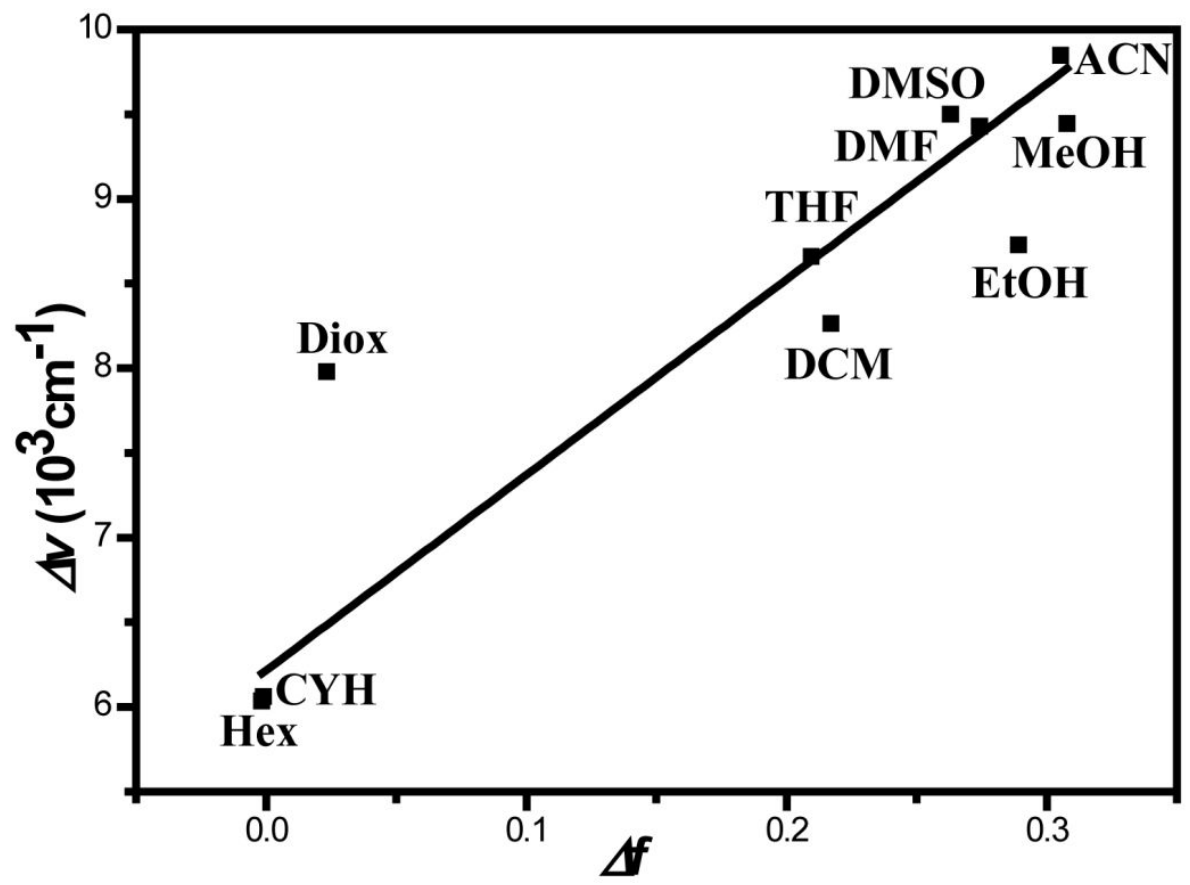

Figure S6. Plots of Stokes shift $(\Delta v)$ of $\left(R_{p}\right) /\left(S_{p}\right)$-3c versus solvent orientation polarizability $(\Delta f)$ in different solvents, ${ }^{2}$ such as hexane (Hex), cyclohexane (CYH), dichloromethane (DCM), tetrahydrofuran (THF), ethanol (EtOH), 1,4-dioxane (Diox), acetonitrile (ACN), N,N-dimethylformamide (DMF), methanol (MeOH), dimethyl sulfoxide (DMSO), respectively. 

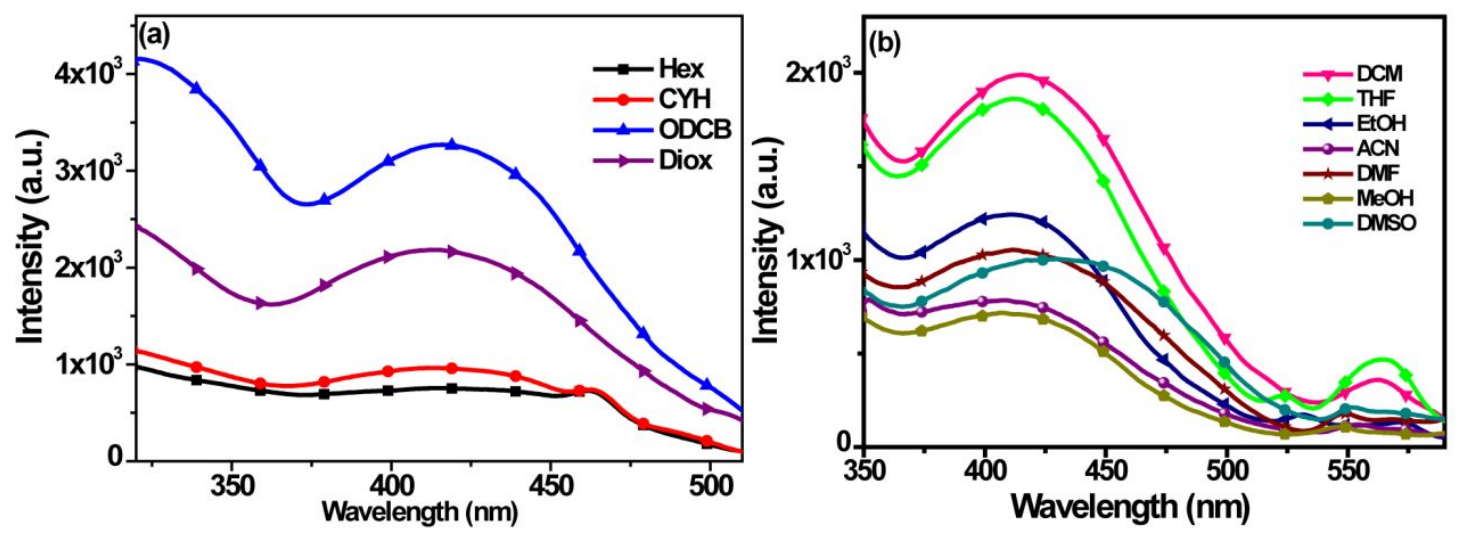

Figure S7. Excitation spectra of $\left(R_{p}\right) /\left(S_{p}\right)-\mathbf{3 c}$ in different solvents, such as hexane (Hex), cyclohexane $(\mathrm{CYH})$, dichloromethane (DCM), tetrahydrofuran (THF), ethanol (EtOH), 1,4-dioxane (Diox), acetonitrile (ACN), $\mathrm{N}, \mathrm{N}$-dimethylformamide (DMF), methanol (MeOH), dimethyl sulfoxide (DMSO), respectively.
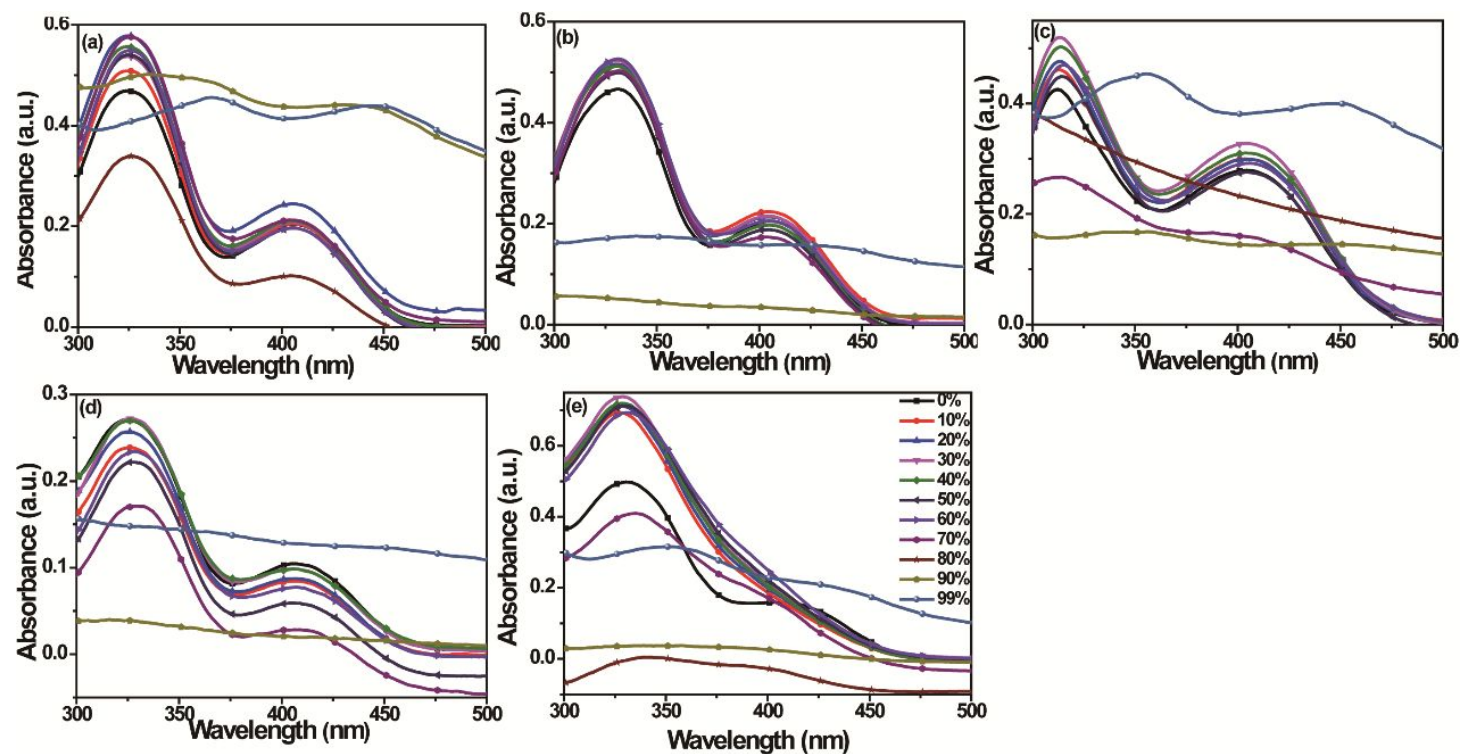

Figure S8. Absorption spectra of compounds 3a (a), 3b (b), 3c (c), 3d (d) and 3e (e) in $\mathrm{THF} / \mathrm{H}_{2} \mathrm{O}$ mixtures $(50$ $\mu \mathrm{M})$ with varied volumetric fractions of water. 

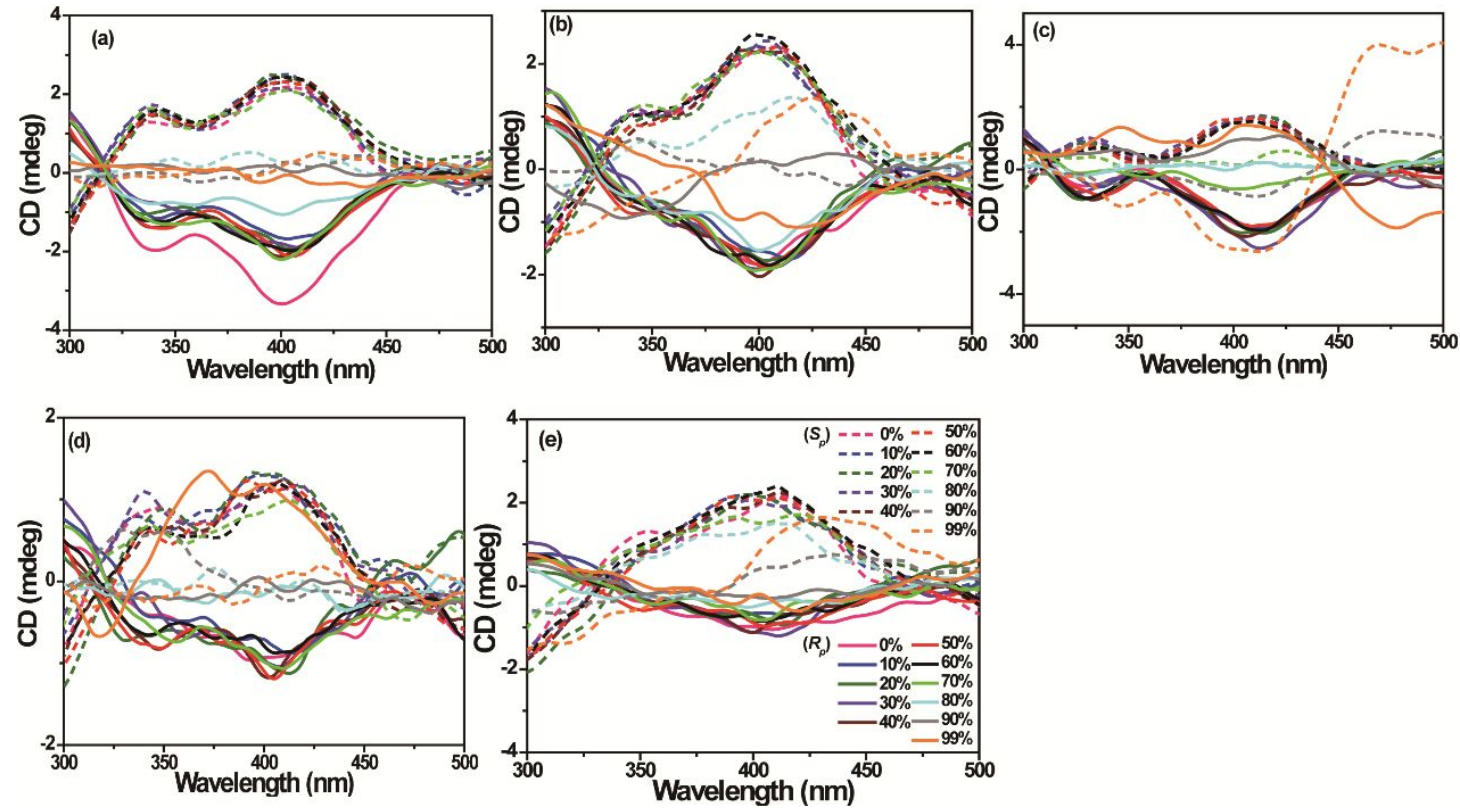

Figure S9. CD spectra of compounds $\left(R_{p}\right) /\left(S_{p}\right)-\mathbf{3 a}(\mathrm{a}),\left(R_{p}\right) /\left(S_{p}\right)-\mathbf{3 b}(\mathrm{b}),\left(R_{p}\right) /\left(S_{p}\right)-\mathbf{3 c}(\mathrm{c}),\left(R_{p}\right) /\left(S_{p}\right)-\mathbf{3 d}(\mathrm{d})$ and $\left(R_{p}\right) /\left(S_{p}\right)-3 \mathrm{e}(\mathrm{e})$ in $\mathrm{THF} / \mathrm{H}_{2} \mathrm{O}$ mixtures $(50 \mu \mathrm{M})$ with varied volumetric fractions of water.
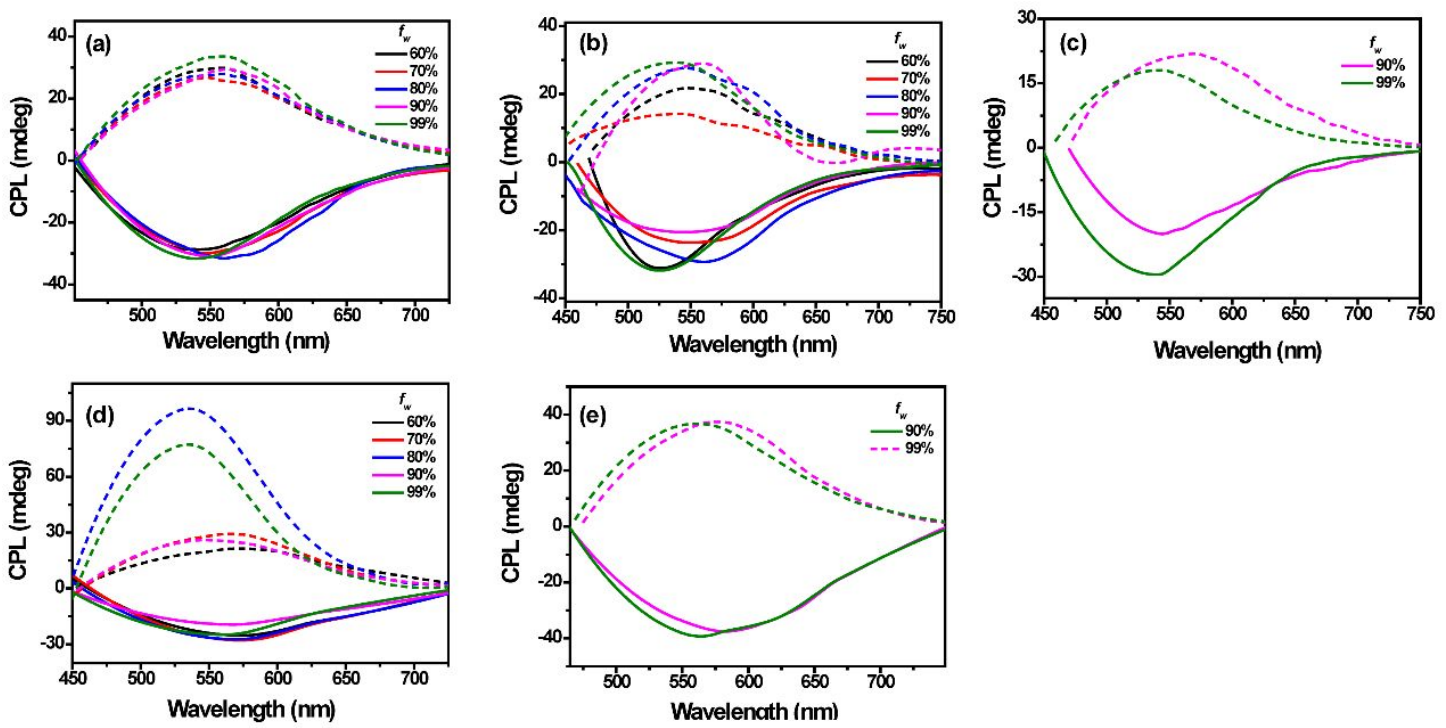

Figure S10. CPL spectra of compounds $\left(R_{p}\right) /\left(S_{p}\right)-\mathbf{3 a}(\mathrm{a}),\left(R_{p}\right) /\left(S_{p}\right)-\mathbf{3 b}(\mathrm{b}),\left(R_{p}\right) /\left(S_{p}\right)-\mathbf{3 c}(\mathrm{c}),\left(R_{p}\right) /\left(S_{p}\right)-\mathbf{3 d}(\mathrm{d})$ and $\left(R_{p}\right) /\left(S_{p}\right)-3 \mathbf{e}(\mathrm{e})$ in $\mathrm{THF} / \mathrm{H}_{2} \mathrm{O}$ mixtures $(50 \mu \mathrm{M})$ with varied volumetric fractions of water. 

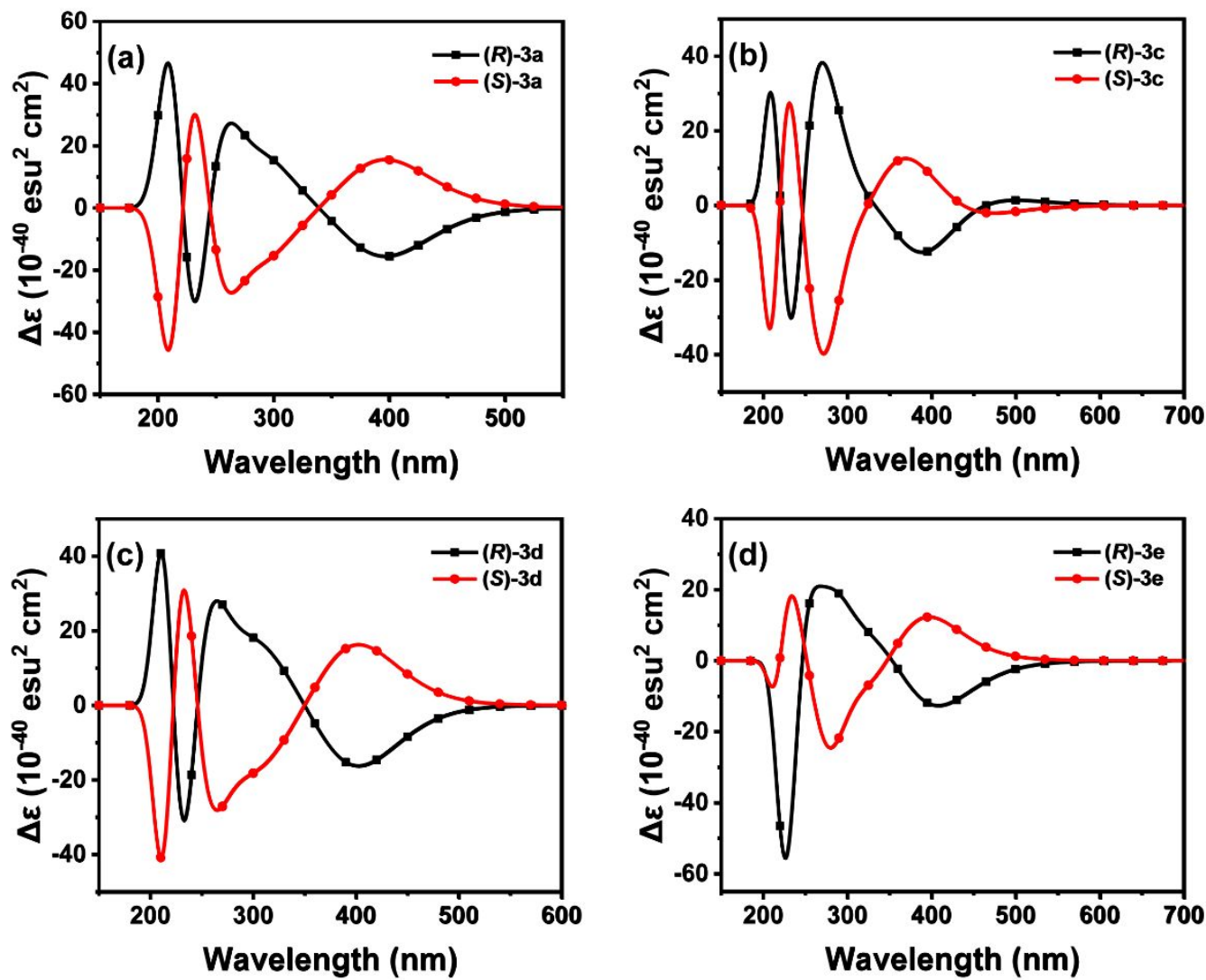

Figure S11. Calculated CD spectra of compounds $\left(R_{p}\right) /\left(S_{p}\right)-\mathbf{3 a}(\mathrm{a}),\left(R_{p}\right) /\left(S_{p}\right)-\mathbf{3 c}(\mathrm{b}),\left(R_{p}\right) /\left(S_{p}\right)-\mathbf{3 d}(\mathrm{c})$ and $\left(R_{p}\right) /\left(S_{p}\right)-\mathbf{3 e}(\mathrm{d})$.
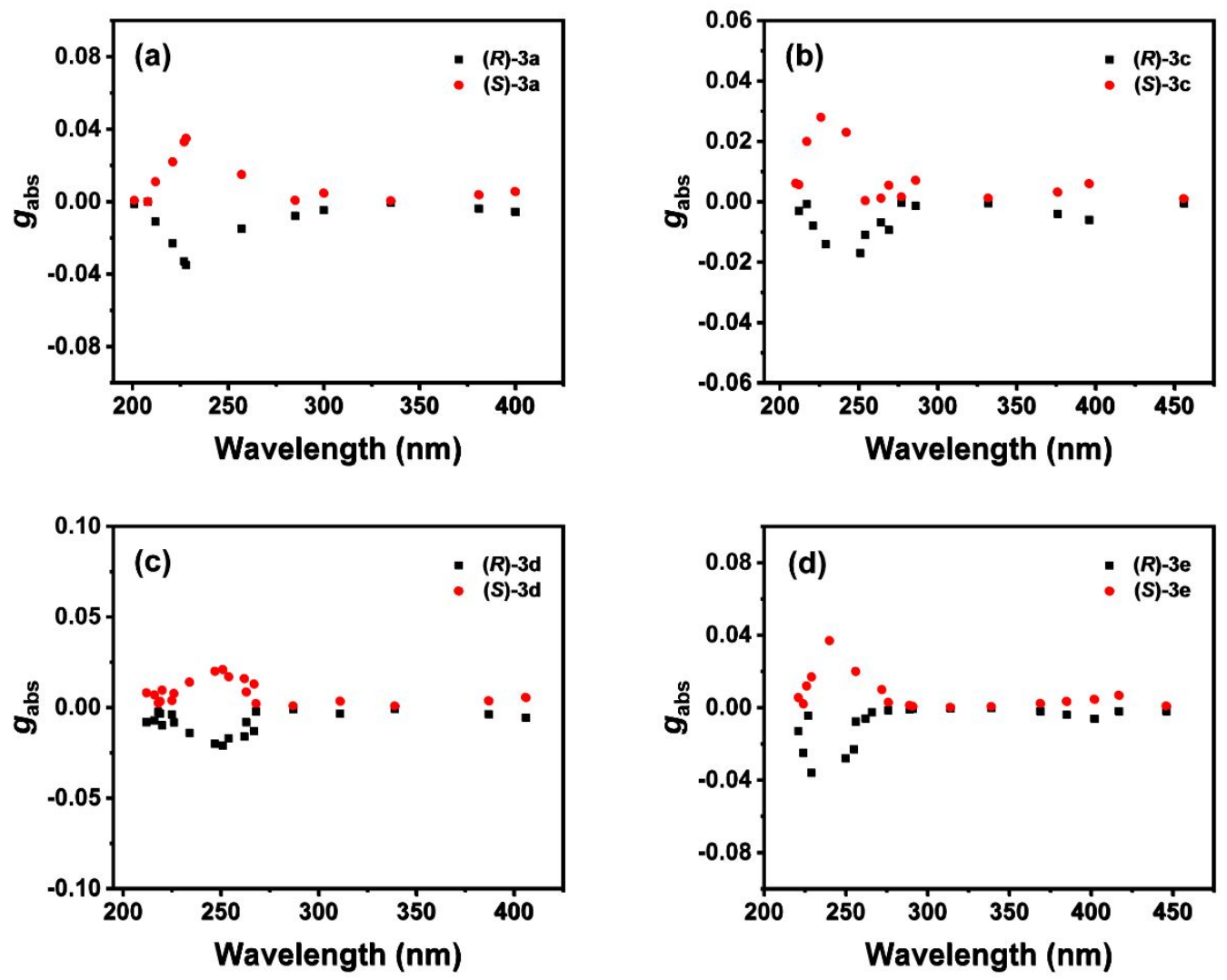
Figure S12. Calculated dissymmetry factor $\left(g_{\mathrm{abs}}\right)$ of compounds $\left(R_{p}\right) /\left(S_{p}\right)-\mathbf{3 a}(\mathrm{a}),\left(R_{p}\right) /\left(S_{p}\right)-\mathbf{3 c}(\mathrm{b}),\left(R_{p}\right) /\left(S_{p}\right)-\mathbf{3 d}(\mathrm{c})$ and $\left(R_{p}\right) /\left(S_{p}\right)-\mathbf{3 e}(\mathrm{d})$.
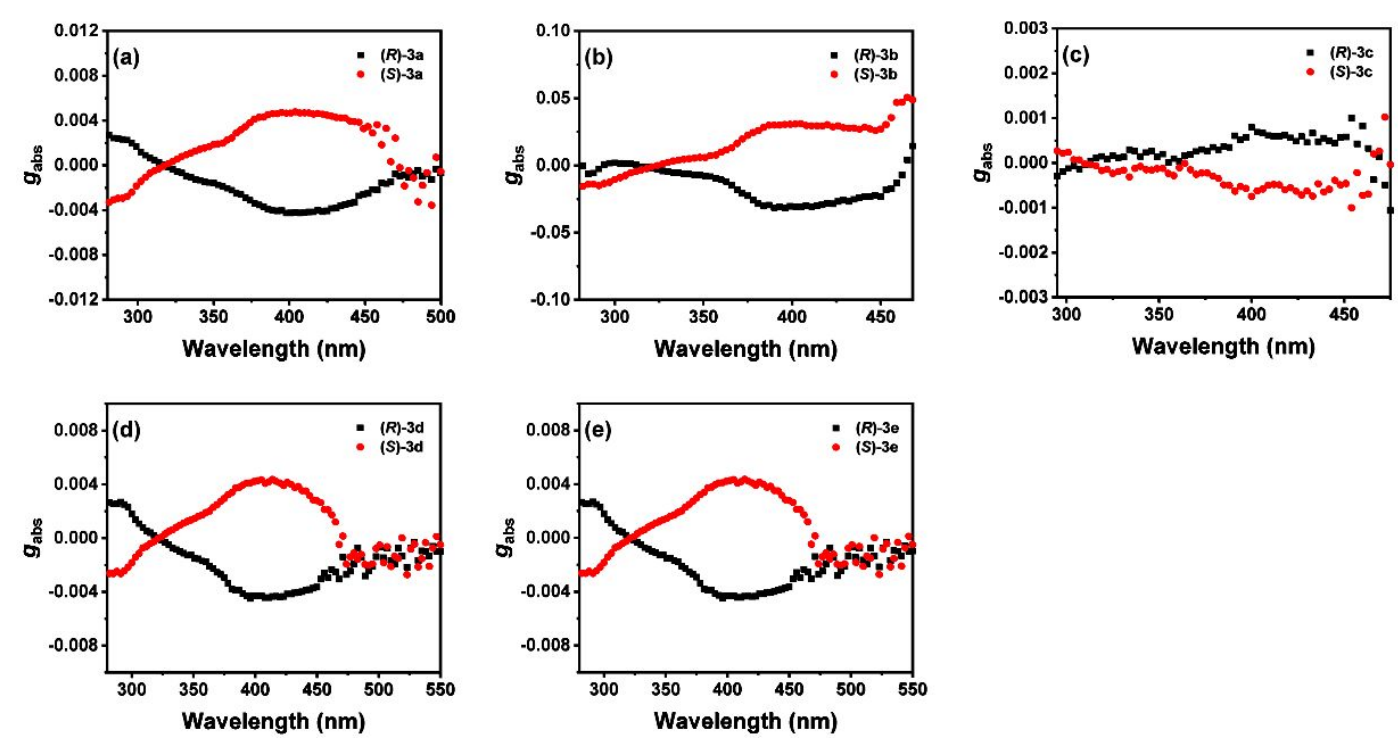

Figure S13. The dissymmetry factor $\left(g_{\text {abs }}\right)$ of compounds $\left(R_{p}\right) /\left(S_{p}\right)-\mathbf{3 a}(\mathrm{a}),\left(R_{p}\right) /\left(S_{p}\right)-\mathbf{3 b}(\mathrm{b}),\left(R_{p}\right) /\left(S_{p}\right)-\mathbf{3 c}(\mathrm{c})$, $\left(R_{p}\right) /\left(S_{p}\right)$-3d $(\mathrm{d})$ and $\left(R_{p}\right) /\left(S_{p}\right)-\mathbf{3 e}(\mathrm{e})$.

Table S1. Related wave functions, oscillator strengths, and calculated electronic excitation energies of $\left(R_{p}\right) /\left(S_{p}\right)$-3a, $,\left(R_{p}\right) /\left(S_{p}\right)-\mathbf{3 c},\left(R_{p}\right) /\left(S_{p}\right) \mathbf{- 3 d}$ and $\left(R_{p}\right) /\left(S_{p}\right)-\mathbf{3 e}$.

\begin{tabular}{|c|c|c|c|c|c|}
\hline Compound & state & $\lambda[\mathrm{nm}]$ & $f$ & $E_{\mathrm{g}}(\mathrm{eV})$ & $\begin{array}{c}\text { Orbital } \\
\text { (coefficient) }\end{array}$ \\
\hline \multirow[t]{3}{*}{$\left(R_{p}\right) /\left(S_{p}\right)-\mathbf{3 a}$} & S1 & 400.75 & 0.0542 & 3.09 & H-L (68.7\%) \\
\hline & S3 & 335.35 & 0.1035 & 3.69 & $\mathrm{H}_{-2}-\mathrm{L}(69.0 \%)$ \\
\hline & S5 & 285.32 & 0.1957 & 4.34 & $\mathrm{H}_{-4}-\mathrm{L}(62.5 \%)$ \\
\hline \multirow[t]{3}{*}{$\left(R_{p}\right) /\left(S_{p}\right)-\mathbf{3 c}$} & S1 & 456.95 & 0.1263 & 2.71 & H-L (70.3\%) \\
\hline & S4 & 332.27 & 0.1160 & 3.73 & $\mathrm{H}_{-3}-\mathrm{L}(68.9 \%)$ \\
\hline & S6 & 286.16 & 0.2837 & 4.33 & $\mathrm{H}_{-5}-\mathrm{L}(58.7 \%)$ \\
\hline \multirow[t]{3}{*}{$\left(R_{p}\right) /\left(S_{p}\right)-\mathbf{3 d}$} & S1 & 406.11 & 0.0549 & 3.05 & H-L (68.4\%) \\
\hline & S3 & 339.61 & 0.1167 & 3.65 & $\mathrm{H}_{-2}-\mathrm{L}(69.2 \%)$ \\
\hline & S5 & 287.09 & 0.2144 & 4.31 & $\mathrm{H}_{-4}-\mathrm{L}(69.1 \%)$ \\
\hline \multirow[t]{3}{*}{$\left(R_{p}\right) /\left(S_{p}\right)-\mathbf{3 e}$} & S1 & 446.83 & 0.0255 & 2.77 & H-L (69.1\%) \\
\hline & S3 & 402.63 & 0.0428 & 3.07 & $\mathrm{H}-\mathrm{L}_{+1}(66.1 \%)$ \\
\hline & S6 & 339.20 & 0.0869 & 3.65 & $\mathrm{H}_{-2}-\mathrm{L}_{+1}(67.7 \%)$ \\
\hline
\end{tabular}

Table S2. Transition Dipole Moments and $g_{\text {abs }}$ values of compounds $\left(R_{p}\right) /\left(S_{p}\right)-\mathbf{3 a},\left(R_{p}\right) /\left(S_{p}\right)-\mathbf{3 c},\left(R_{p}\right) /\left(S_{p}\right)-\mathbf{3 d}$ and

\begin{tabular}{cccccc}
\multicolumn{5}{c}{$\left(R_{p}\right) /\left(S_{p}\right)-\mathbf{3 e} .^{a}$} \\
\hline Compound & $\begin{array}{c}\mathrm{b}|\mu|\left(10^{-18}\right. \\
\mathrm{esu} \cdot \mathrm{cm})\end{array}$ & $\begin{array}{c}\mathrm{c}|m|\left(10^{-21}\right. \\
\left.\mathrm{erg} \cdot \mathrm{G}^{-1}\right)\end{array}$ & ${ }^{\mathrm{d}} \cos \theta_{\mu, m}$ & \multicolumn{2}{c}{$g_{\text {abs, dichloromethane }}$} \\
\hline & & & & Theoretical & Experimental \\
$\left(R_{p}\right)-\mathbf{3 a}$ & 2.15 & 8.27 & 0.3641 & $5.6 \times 10^{-3}$ & $-5.6 \times 10^{-3}$ \\
$\left(S_{p}\right)-\mathbf{3 a}$ & 2.15 & 8.27 & -0.3642 & $-5.6 \times 10^{-3}$ & $5.1 \times 10^{-3}$
\end{tabular}




$\begin{array}{lccccc}\left(R_{p}\right)-\mathbf{3 b} & - & - & - & - & -6.1 \times 10^{-3} \\ \left(S_{p}\right)-\mathbf{3 b} & - & - & - & - & 6.2 \times 10^{-3} \\ \left(R_{p}\right)-\mathbf{3 c} & 3.50 & 3.28 & -0.1447 & 1.0 \times 10^{-3} & -1.8 \times 10^{-3} \\ \left(S_{p}\right)-\mathbf{3 c} & 3.00 & 2.51 & -0.3014 & 1.0 \times 10^{-3} & 1.9 \times 10^{-3} \\ \left(R_{p}\right)-\mathbf{3 d} & 2.18 & 9.01 & 0.3357 & 5.5 \times 10^{-3} & -3.1 \times 10^{-3} \\ \left(S_{p}\right)-\mathbf{3 d} & 2.18 & 9.00 & -0.3362 & -5.6 \times 10^{-3} & 3.1 \times 10^{-3} \\ \left(R_{p}\right)-\mathbf{3 e} & 1.56 & 3.23 & 0.2352 & 2.0 \times 10^{-3} & -4.1 \times 10^{-3} \\ \left(S_{p}\right)-\mathbf{3 e} & 1.21 & 2.38 & -0.1129 & -1.0 \times 10^{-3} & 4.6 \times 10^{-3}\end{array}$

${ }^{a}$ TD-DFT calculation at the B3LYP/6-31G(d,p) level with the self-consistent field method (dichloromethane) using the excited-state structures. ${ }^{b}$ The electric transition moment. ${ }^{c}$ The magnetic transition moment. ${ }^{d} \theta_{\mu, m}$ is angle of vectors between $\mu$ and $m$.

Table S3. Transition Dipole Moments and $g_{\text {lum }}$ values of compounds $\left(R_{p}\right) /\left(S_{p}\right)-\mathbf{3 a},\left(R_{p}\right) /\left(S_{p}\right)-\mathbf{3 c},\left(R_{p}\right) /\left(S_{p}\right)-\mathbf{3 d}$ and

\begin{tabular}{cccccc}
\multicolumn{5}{c}{$\left(R_{p}\right) /\left(S_{p}\right)-\mathbf{3 e}{ }^{a}$} \\
\hline Compound & $\begin{array}{c}\mathrm{b}|\mu|\left(10^{-18}\right. \\
\mathrm{esu} \cdot \mathrm{cm})\end{array}$ & $\begin{array}{c}\mathrm{c}|m|\left(10^{-21}\right. \\
\left.\mathrm{erg} \cdot \mathrm{G}^{-1}\right)\end{array}$ & ${ }^{\mathrm{d}} \cos \theta_{\mu, m}$ & \multicolumn{2}{c}{$g_{\text {lum, dichloromethane }}$} \\
\hline & & & & Theoretical & Experimental \\
$\left(R_{p}\right)-3 \mathbf{a}$ & 2.42 & 6.88 & 0.2046 & $2.3 \times 10^{-3}$ & $-3.6 \times 10^{-3}$ \\
$\left(S_{p}\right)-3 \mathbf{a}$ & 2.42 & 6.88 & -0.2048 & $-2.3 \times 10^{-3}$ & $4.5 \times 10^{-3}$ \\
$\left(R_{p}\right)-3 \mathbf{b}$ & - & - & - & - & $-6.2 \times 10^{-3}$ \\
$\left(S_{p}\right)-3 \mathbf{b}$ & - & - & - & - & $5.6 \times 10^{-3}$ \\
$\left(R_{p}\right)-3 \mathbf{c}$ & 1.15 & 1.50 & 0.8756 & $4.6 \times 10^{-3}$ & - \\
$\left(S_{p}\right)-3 \mathbf{c}$ & 1.52 & 1.52 & 0.8715 & $3.5 \times 10^{-3}$ & - \\
$\left(R_{p}\right)-3 \mathbf{d}$ & 2.43 & 7.59 & -0.0019 & $-2.4 \times 10^{-5}$ & $-5.4 \times 10^{-3}$ \\
$\left(S_{p}\right)-3 \mathbf{d}$ & 2.44 & 7.59 & -0.1919 & $-2.4 \times 10^{-3}$ & $3.6 \times 10^{-3}$ \\
$\left(R_{p}\right)-3 \mathbf{e}$ & 1.88 & 1.75 & 0.0103 & $3.8 \times 10^{-5}$ & $-5.6 \times 10^{-3}$ \\
$\left(S_{p}\right)-3 \mathbf{e}$ & 1.74 & 1.67 & 0.0442 & $1.7 \times 10^{-4}$ & $6.2 \times 10^{-3}$ \\
\hline
\end{tabular}

${ }^{a}$ TD DFT calculation at the B3LYP/6-31G(d,p) level with the self-consistent field method (dichloromethane) using the excited-state structures. ${ }^{b}$ The electric transition moment. ${ }^{c}$ The magnetic transition moment. ${ }^{d} \theta_{\mu, m}$ is angle of vectors between $\mu$ and $m$. 
Table S4. Photophysical data of $\left(R_{p}\right) /\left(S_{p}\right)$-3a in various solvents.

\begin{tabular}{lcccc}
\hline Solvent & $\lambda_{\text {abs }}(\mathrm{nm})$ & $\varepsilon\left(\mathrm{mol}^{-1} \mathrm{~cm}^{-1}\right)$ & $\lambda_{\text {em }}(\mathrm{nm})$ & $\begin{array}{c}\text { Stokes Shift } \\
\left(\mathrm{nm} / \mathrm{cm}^{-1}\right)\end{array}$ \\
\hline Hexane & $323 / 409$ & $7483 / 3340$ & 531 & $122 / 5617$ \\
Cyclohexane & $323 / 410$ & $8351 / 3737$ & 532 & $122 / 5593$ \\
1,2-Dichlorobenzene & $326 / 410$ & $7739 / 3345$ & 545 & $135 / 6042$ \\
Dichloromethane & $325 / 407$ & $6922 / 2605$ & 543 & $136 / 6154$ \\
Tetrahydrofuran & $323 / 406$ & $8313 / 3418$ & 540 & $134 / 6112$ \\
Ethanol & $325 / 406$ & $9505 / 3581$ & 545 & $139 / 6282$ \\
$1,4-$ Dioxane & $322 / 406$ & $7003 / 2981$ & 539 & $133 / 6078$ \\
Acetonitrile & $324 / 403$ & $8921 / 3188$ & 547 & $144 / 6532$ \\
$N, N$-dimethylformamide & $325 / 403$ & $8788 / 3291$ & 547 & $144 / 6532$ \\
Methanol & $325 / 403$ & $8722 / 3183$ & 545 & $142 / 6465$ \\
Dimethyl sulfoxide & $325 / 403$ & $8782 / 3258$ & 550 & $147 / 6632$ \\
\hline
\end{tabular}


Table S5. Photophysical data of $\left(R_{p}\right) /\left(S_{p}\right)$-3b in various solvents.

\begin{tabular}{lcccc}
\hline Solvent & $\lambda_{\text {abs }}(\mathrm{nm})$ & $\varepsilon\left(\mathrm{mol}^{-1} \mathrm{~cm}^{-1}\right)$ & $\lambda_{\text {em }}(\mathrm{nm})$ & $\begin{array}{c}\text { Stokes Shift } \\
\left(\mathrm{nm} / \mathrm{cm}^{-1}\right)\end{array}$ \\
\hline Hexane & $330 / 408$ & $5260 / 2400$ & 522 & $114 / 5353$ \\
Cyclohexane & $330 / 410$ & $5000 / 2340$ & 523 & $113 / 5270$ \\
1,2-Dichlorobenzene & $334 / 409$ & $5680 / 2540$ & 532 & $123 / 5653$ \\
Dichloromethane & $331 / 406$ & $5380 / 2140$ & 532 & $127 / 5834$ \\
Tetrahydrofuran & $329 / 405$ & $5420 / 2360$ & 529 & $124 / 5788$ \\
Ethanol & $331 / 404$ & $5780 / 2240$ & 534 & $130 / 6026$ \\
$1,4-$ Dioxane & $329 / 405$ & $5200 / 2300$ & 531 & $126 / 5859$ \\
Acetonitrile & $328 / 401$ & $5720 / 2140$ & 535 & $134 / 6246$ \\
$N, N$-dimethylformamide & $329 / 402$ & $5480 / 2180$ & 535 & $133 / 6184$ \\
Methanol & $329 / 403$ & $5600 / 2140$ & 532 & $129 / 6017$ \\
Dimethyl sulfoxide & $330 / 402$ & $6500 / 2540$ & 535 & $133 / 6184$ \\
\hline
\end{tabular}


Table S6. Photophysical data of $\left(R_{p}\right) /\left(S_{p}\right)$-3c in various solvents.

\begin{tabular}{lcccc}
\hline Solvent & $\lambda_{\text {abs }}(\mathrm{nm})$ & $\varepsilon\left(\mathrm{mol}^{-1} \mathrm{~cm}^{-1}\right)$ & $\lambda_{\text {em }}(\mathrm{nm})$ & $\begin{array}{c}\text { Stokes Shift } \\
\left(\mathrm{nm} / \mathrm{cm}^{-1}\right)\end{array}$ \\
\hline Hexane & $313 / 404$ & $9467 / 6448$ & 535 & $131 / 6061$ \\
Cyclohexane & $313 / 405$ & $10709 / 7268$ & 536 & $131 / 6035$ \\
1,2-Dichlorobenzene & $316 / 410$ & $10536 / 7001$ & 594 & $184 / 7555$ \\
Dichloromethane & $315 / 406$ & $9422 / 5662$ & 611 & $205 / 8264$ \\
Tetrahydrofuran & $313 / 403$ & $11983 / 7865$ & 619 & $216 / 8659$ \\
Ethanol & $314 / 404$ & $11954 / 7398$ & 624 & $220 / 8727$ \\
1,4-Dioxane & $313 / 403$ & $10851 / 7077$ & 594 & $191 / 7979$ \\
Acetonitrile & $313 / 400$ & $12274 / 7019$ & 660 & $260 / 9848$ \\
$N, N$-dimethylformamide & $314 / 403$ & $11467 / 7059$ & 650 & $247 / 9429$ \\
Methanol & $315 / 402$ & $10786 / 6500$ & 648 & $246 / 9444$ \\
Dimethyl sulfoxide & $315 / 403$ & $11327 / 6786$ & 653 & $250 / 9500$ \\
\hline
\end{tabular}


Table S7. Photophysical data of $\left(R_{p}\right) /\left(S_{p}\right)$-3d in various solvents.

\begin{tabular}{lcccc}
\hline Solvent & $\lambda_{\text {abs }}(\mathrm{nm})$ & $\varepsilon\left(\mathrm{mol}^{-1} \mathrm{~cm}^{-1}\right)$ & $\lambda_{e m}(\mathrm{~nm})$ & $\begin{array}{c}\text { Stokes Shift } \\
\left(\mathrm{nm} / \mathrm{cm}^{-1}\right)\end{array}$ \\
\hline Hexane & $327 / 412$ & $8577 / 3588$ & 543 & $131 / 5856$ \\
Cyclohexane & $328 / 413$ & $10426 / 4417$ & 541 & $128 / 5729$ \\
1,2-Dichlorobenzene & $331 / 413$ & $8817 / 3423$ & 559 & $146 / 6324$ \\
Dichloromethane & $329 / 410$ & $9657 / 3454$ & 560 & $150 / 6533$ \\
Tetrahydrofuran & $327 / 408$ & $11065 / 4238$ & 558 & $150 / 6589$ \\
Ethanol & $328 / 408$ & $114433 / 4094$ & 562 & $154 / 6716$ \\
1,4-Dioxane & $326 / 408$ & $12860 / 5005$ & 552 & $144 / 6394$ \\
Acetonitrile & $326 / 404$ & $9469 / 3200$ & 566 & $162 / 7085$ \\
$N, N$-dimethylformamide & $321 / 405$ & $10660 / 3802$ & 568 & $163 / 7086$ \\
Methanol & $327 / 406$ & $9275 / 3241$ & 558 & $152 / 6709$ \\
Dimethyl sulfoxide & $327 / 405$ & $12803 / 4405$ & 569 & $164 / 7117$ \\
\hline
\end{tabular}


Table S8. Photophysical data of $\left(R_{p}\right) /\left(S_{p}\right)-3 \mathbf{e}$ in various solvents.

\begin{tabular}{lcccc}
\hline Solvent & $\lambda_{\text {abs }}(\mathrm{nm})$ & $\varepsilon\left(\mathrm{mol}^{-1} \mathrm{~cm}^{-1}\right)$ & $\lambda_{e m}(\mathrm{~nm})$ & $\begin{array}{c}\text { Stokes Shift } \\
\left(\mathrm{nm} / \mathrm{cm}^{-1}\right)\end{array}$ \\
\hline Hexane & $330 / 417$ & $10600 / 4200$ & 581 & $164 / 6769$ \\
Cyclohexane & $331 / 417$ & $10800 / 4240$ & 581 & $164 / 6769$ \\
1,2-Dichlorobenzene & $337 / 416$ & $11720 / 4360$ & 604 & $188 / 7482$ \\
Dichloromethane & $334 / 413$ & $11620 / 3820$ & 608 & $195 / 7766$ \\
Tetrahydrofuran & $331 / 410$ & $11380 / 4740$ & 603 & $193 / 7970$ \\
Ethanol & $332 / 410$ & $13480 / 4440$ & 609 & $199 / 7970$ \\
1,4-Dioxane & $329 / 410$ & $12460 / 4460$ & 593 & $183 / 7527$ \\
Acetonitrile & $330 / 406$ & $12920 / 4000$ & 629 & $223 / 8732$ \\
$N, N$-dimethylformamide & $330 / 406$ & $9500 / 2940$ & 625 & $219 / 8631$ \\
Methanol & $331 / 408$ & $13080 / 4160$ & 623 & $215 / 8458$ \\
Dimethyl sulfoxide & $331 / 407$ & $13440 / 4460$ & 625 & $218 / 8570$ \\
\hline
\end{tabular}


$\langle$ Chromatogram〉

$\mathrm{mV}$

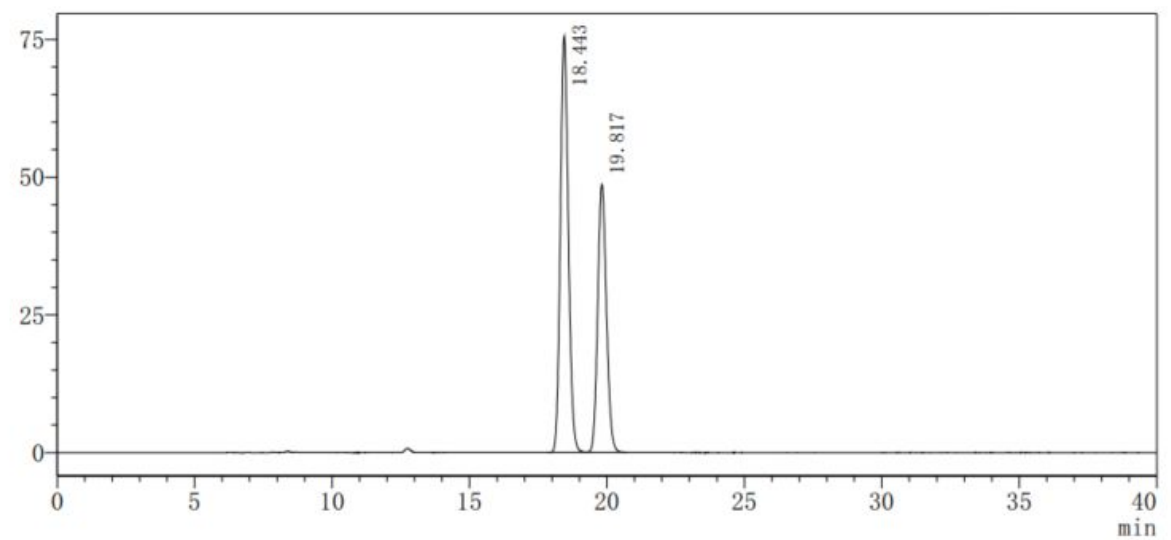

$\langle$ Peak Table〉

PDA Ch1 410nm

\begin{tabular}{|r|r|c|c|r|}
\hline Peak Name & Retention Time & Area & Peak Height & Area \% \\
\hline 1 & 18.443 & 1536337 & 75458 & 58.997 \\
\hline 2 & 19.817 & 1067738 & 48672 & 41.003 \\
\hline Total & & 2604074 & 124130 & 100.000 \\
\hline
\end{tabular}

Figure S14. Chromatogram of the racemic of 3a.

〈Chromatogram〉

$\mathrm{mV}$

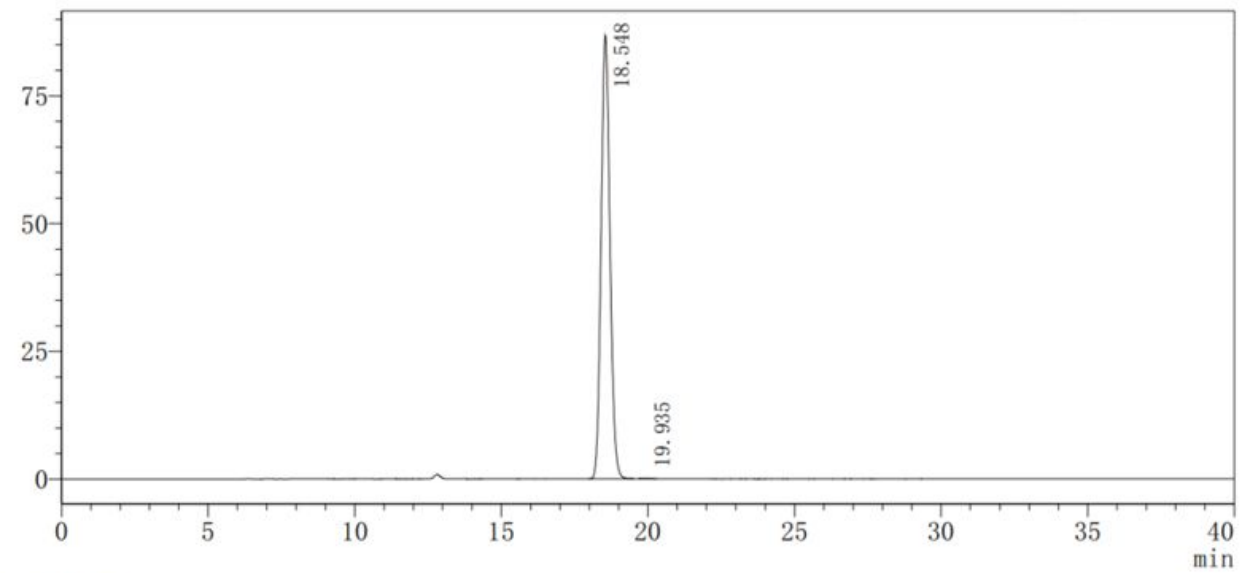

$\langle$ Peak Table $\rangle$

PDA Ch1 410nm

\begin{tabular}{|r|r|r|r|r|}
\hline Peak Name & Retention Time & Area & Peak Height & Area \% \\
\hline 1 & 18.548 & 1828008 & 86739 & 99.930 \\
\hline 2 & 19.935 & 1282 & 67 & 0.070 \\
\hline Total & & 1829290 & 86806 & 100.000 \\
\hline
\end{tabular}

Figure S15. Chromatograms of $\left(R_{\mathrm{p}}\right)$-3a. 
〈Chromatogram〉

$\mathrm{mV}$

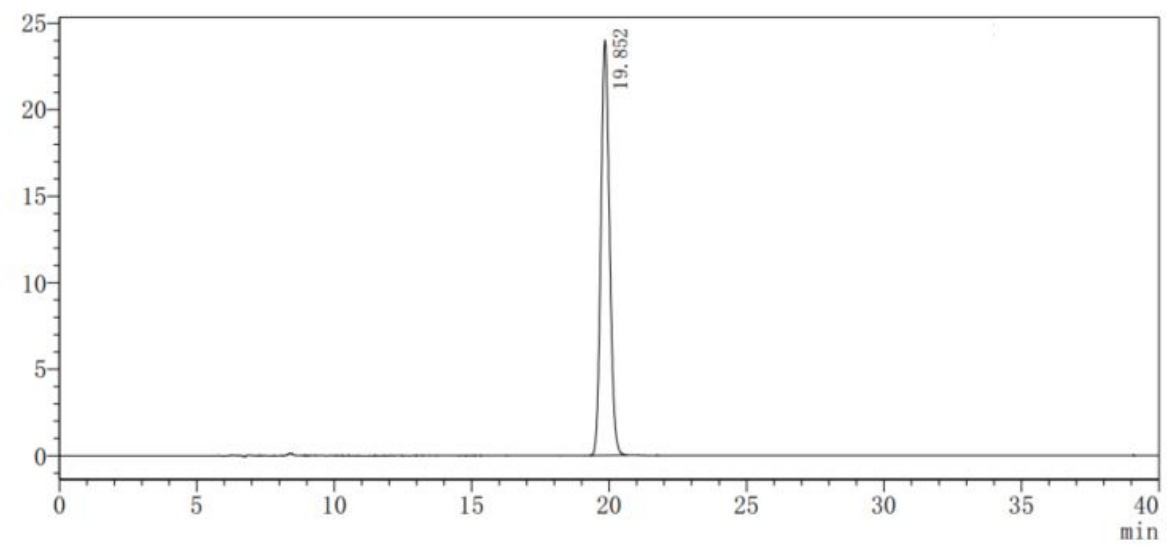

$\langle$ Peak Table $\rangle$

PDA Ch1 410nm

\begin{tabular}{|r|r|r|r|r|}
\hline Peak Name & Retention Time & Area & Peak Height & Area \% \\
\hline 1 & 19.852 & 523094 & 23981 & 100.000 \\
\hline Total & & 523094 & 23981 & 100.000 \\
\hline
\end{tabular}

Figure S16. Chromatogram of $\left(S_{\mathrm{p}}\right)$-3a.

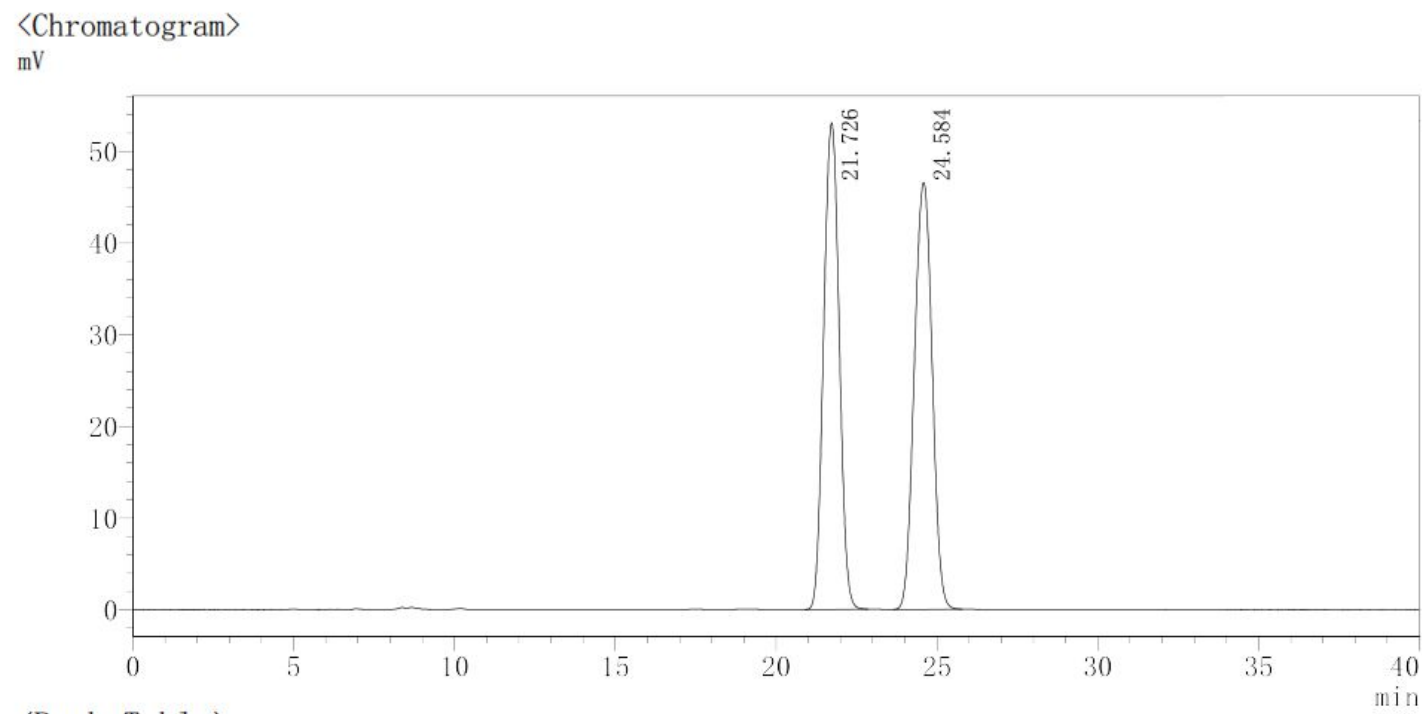

$\langle$ Peak Table $\rangle$

PDA Ch1 410nm

\begin{tabular}{|r|c|c|c|c|}
\hline Peak Name & Retention Time & Area & Peak Height & Area \% \\
\hline 1 & 21.726 & 1781745 & 53057 & 49.818 \\
\hline 2 & 24.584 & 1794745 & 46568 & 50.182 \\
\hline Total & & 3576490 & 99625 & 100.000 \\
\hline
\end{tabular}

Figure S17. Chromatogram of the racemic of $\mathbf{3 b}$. 
〈Chromatogram〉

$\mathrm{mV}$

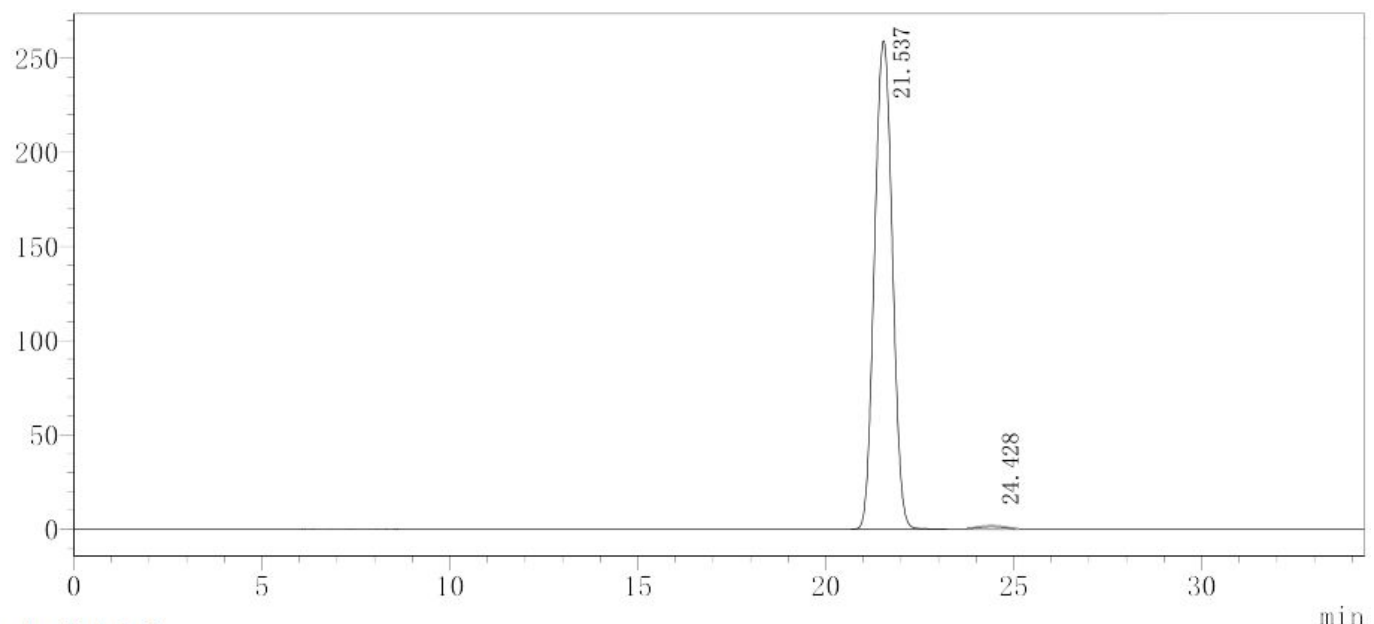

$\langle$ Peak Table $\rangle$

PDA Ch1 410nm

\begin{tabular}{|r|r|r|r|r|}
\hline Peak Name & Retention Time & \multicolumn{1}{|c|}{ Area } & Peak Height & Area \% \\
\hline 1 & 21.537 & 8570854 & 259120 & 99.717 \\
\hline 2 & 24.428 & 24367 & 1559 & 0.283 \\
\hline Total & & 8595221 & 260679 & 100.000 \\
\hline
\end{tabular}

Figure S18. Chromatogram of $\left(R_{\mathrm{p}}\right)-\mathbf{3 b}$.

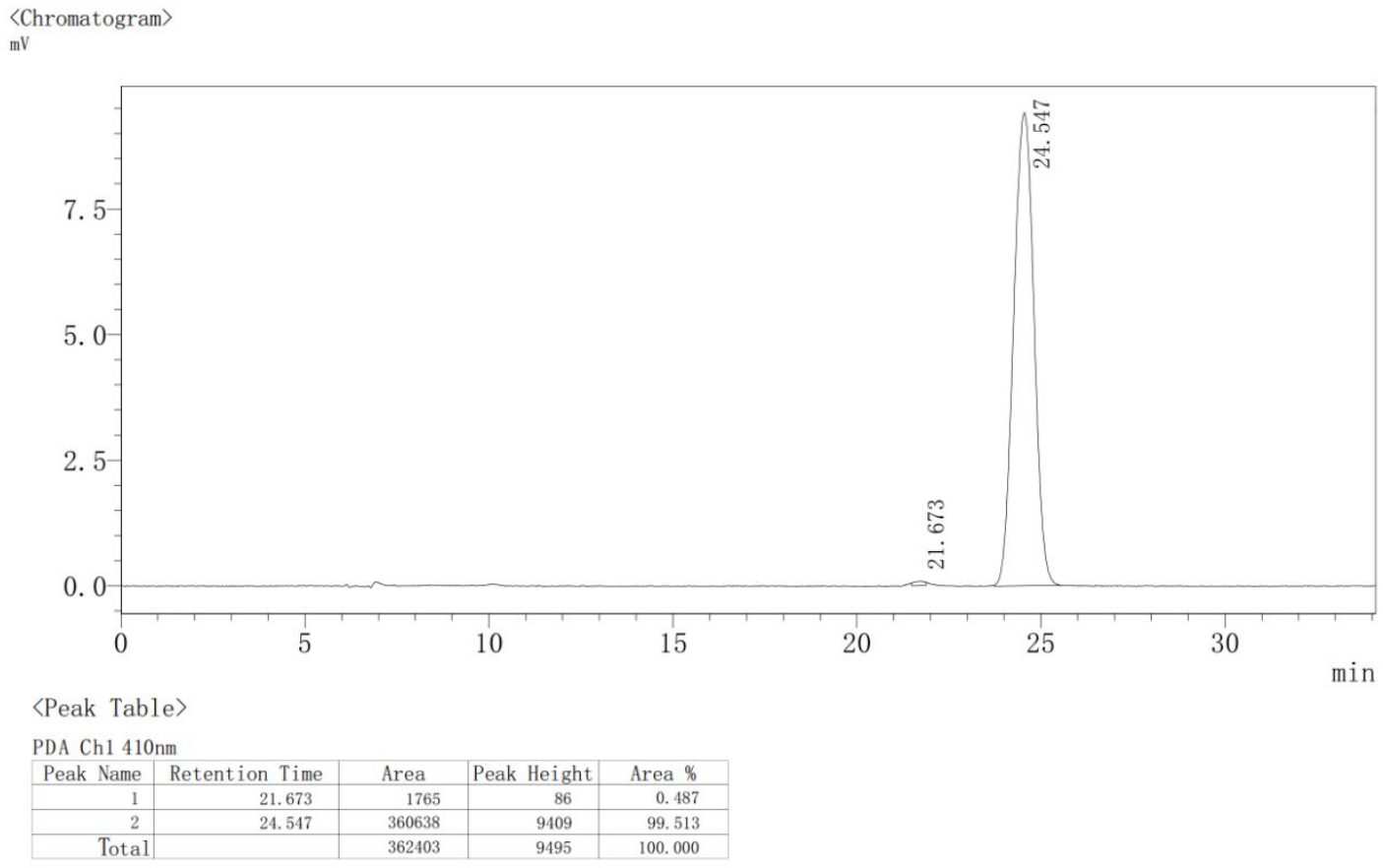

Figure S19. Chromatogram of $\left(S_{\mathrm{p}}\right)$-3b. 
$\langle$ Chromatogram〉

$\mathrm{mV}$

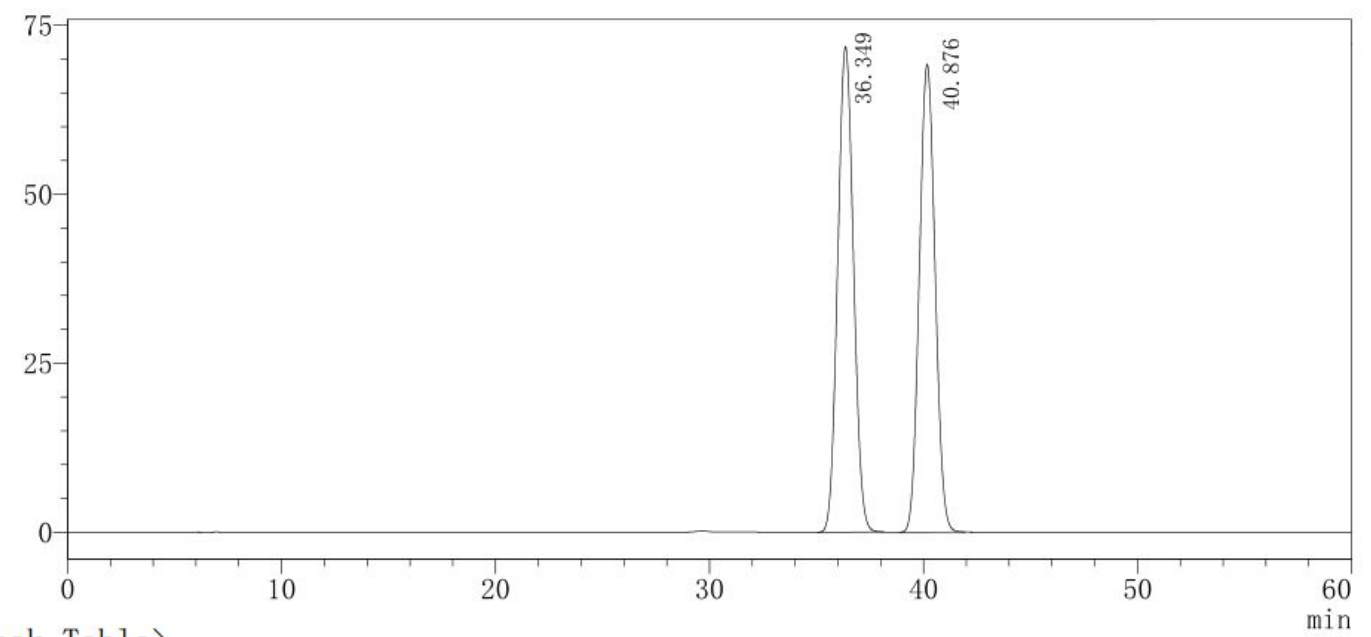

$\langle$ Peak Table $\rangle$

PDA Ch1 410nm

\begin{tabular}{|r|r|c|c|r|}
\hline Peak Name & Retention Time & Area & Peak Height & Area \% \\
\hline 1 & 36.349 & 3665756 & 71800 & 53.248 \\
\hline 2 & 40.876 & 3218551 & 69524 & 46.752 \\
\hline Total & & & & 100.000 \\
\hline
\end{tabular}

Figure S20. Chromatogram of the racemic of 3c.

〈Chromatogram〉

$\mathrm{mV}$

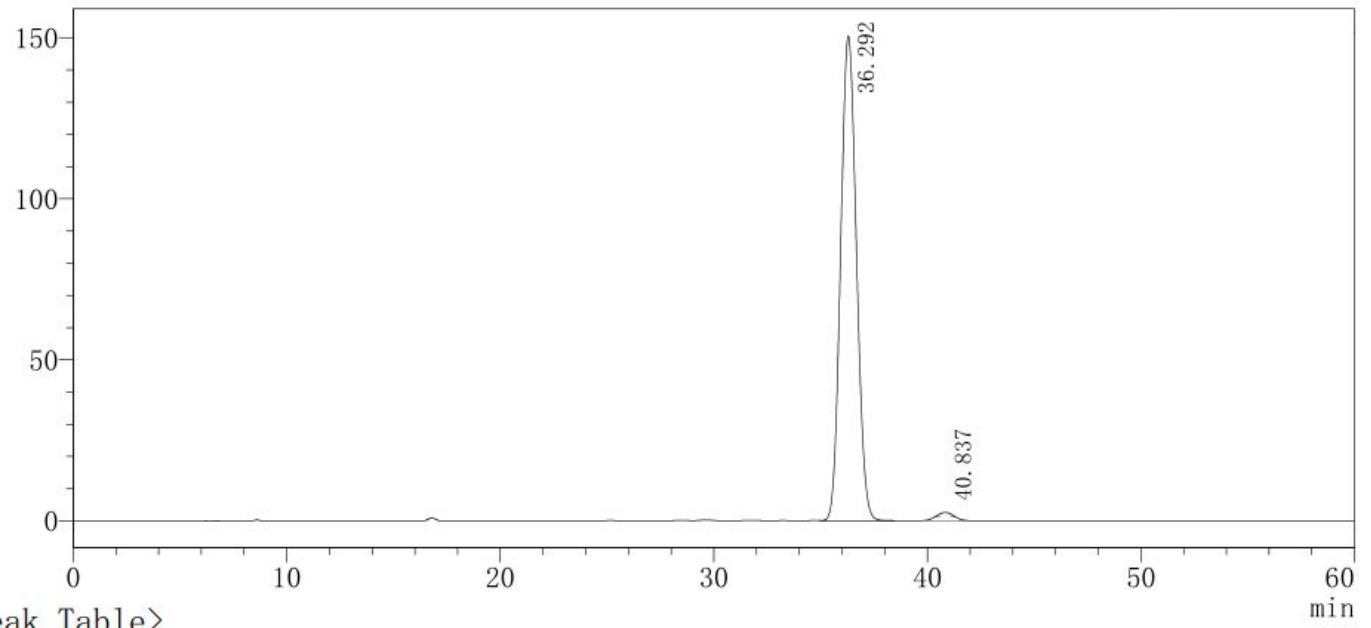

$\langle$ Peak Table $\rangle$

PDA Ch1 410nm

\begin{tabular}{|r|r|r|r|r|}
\hline Peak Name & Retention Time & Area & Peak Height & \multicolumn{1}{c|}{ Area \% } \\
\hline 1 & 36.292 & 7682906 & 150505 & 99.523 \\
\hline 2 & 40.837 & 36826 & 1133 & 0.477 \\
\hline Total & & 7719732 & 151638 & 100.000 \\
\hline
\end{tabular}

Figure S21. Chromatogram of $\left(R_{\mathrm{p}}\right)-\mathbf{3 c}$. 
$\langle$ Chromatogram〉

$\mathrm{mV}$

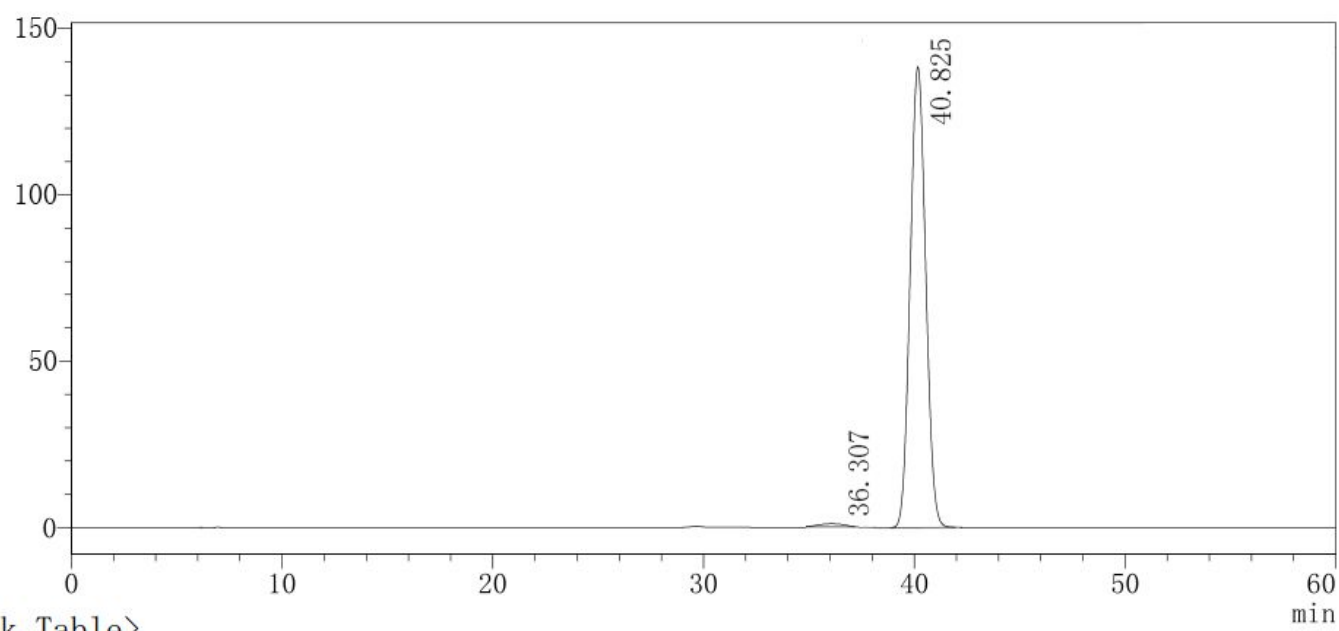

〈Peak Table $\rangle$

PDA Ch1 410nm

\begin{tabular}{|r|c|c|c|c|}
\hline Peak Name & Retention Time & Area & Peak Height & Area \% \\
\hline 1 & 36.307 & 1770 & 192 & 0.184 \\
\hline 2 & 40.825 & 959917 & 14024 & 99.819 \\
\hline Total & & 961687 & 14216 & 100.000 \\
\hline
\end{tabular}

Figure S22. Chromatogram of $\left(S_{\mathrm{p}}\right)$-3c.

$\langle$ Chromatogram

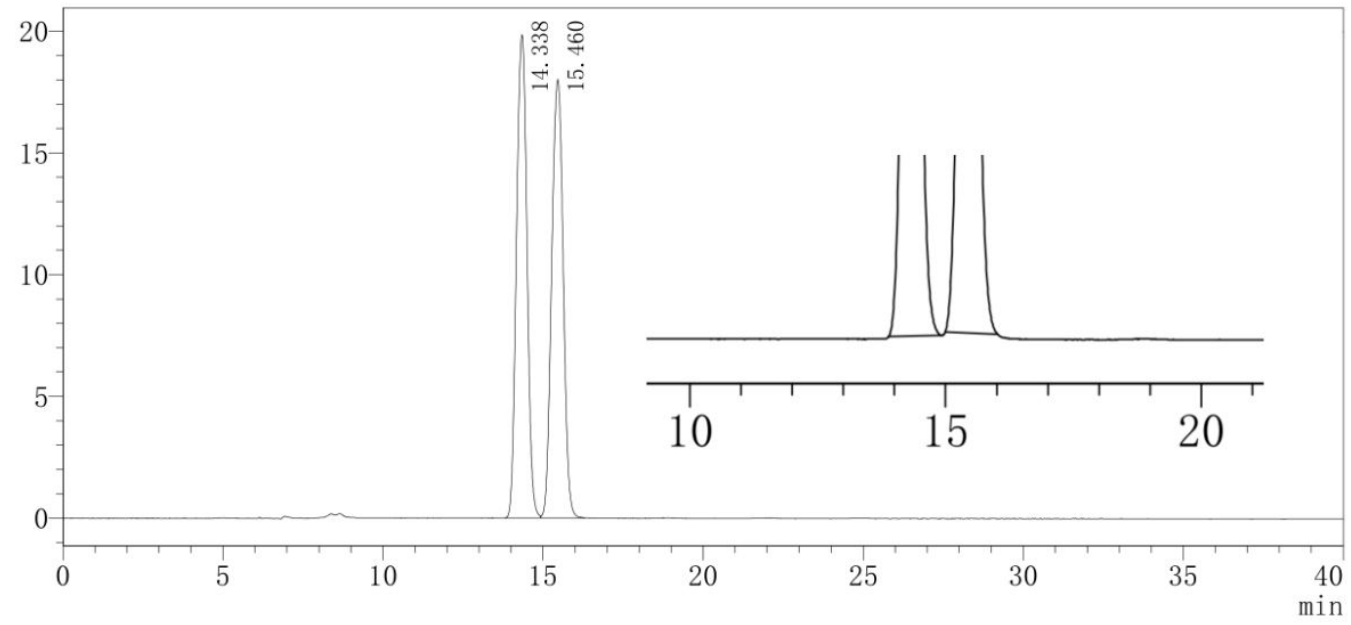

〈Peak Table〉

PDA Ch1 410nm

\begin{tabular}{|r|c|c|c|r|}
\hline Peak Name & Retention Time & Area & Peak Height & Area \% \\
\hline 1 & 14.338 & 451798 & 19834 & 49.920 \\
\hline 2 & 15.460 & 453241 & 17997 & 50.080 \\
\hline Total & & 905039 & 37831 & 100.000 \\
\hline
\end{tabular}

Figure S23. Chromatogram of the racemic of 3d. 
$\langle$ Chromatogram〉

$\mathrm{mV}$

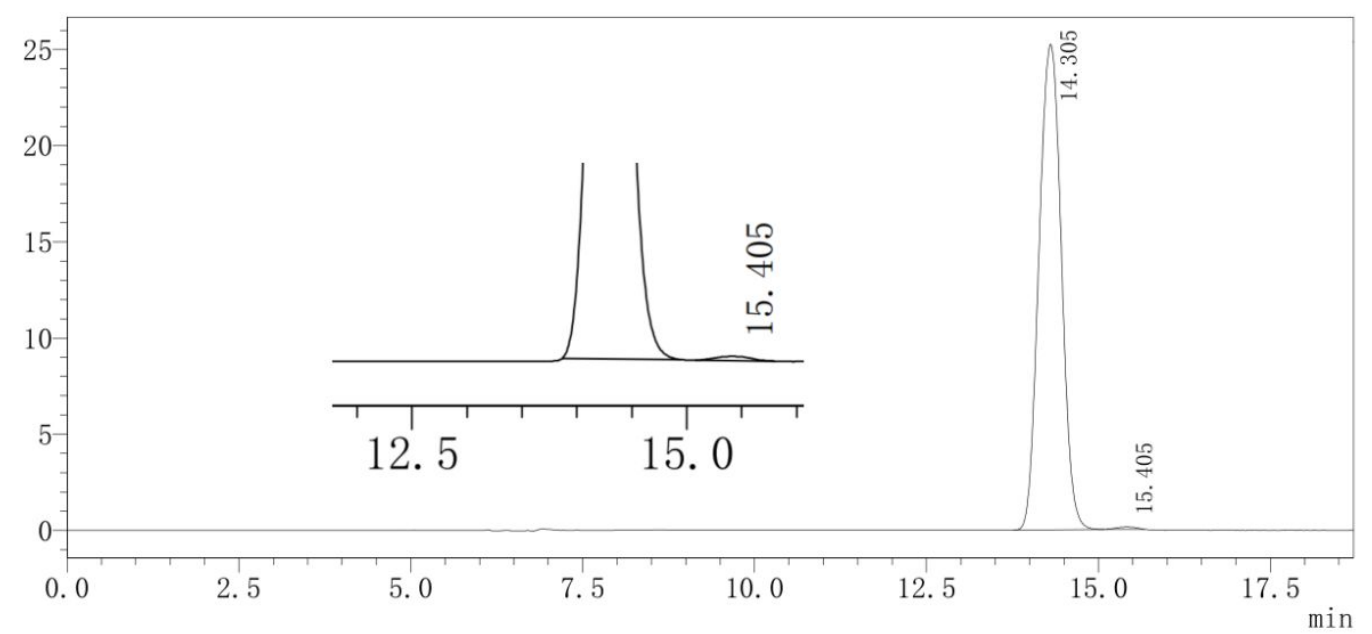

〈Peak Table〉

PDA Ch1 410nm

\begin{tabular}{|r|r|r|r|r|}
\hline Peak Name & Retention Time & Area & Peak Height & Area \% \\
\hline 1 & 14.305 & 570638 & 25227 & 99.574 \\
\hline 2 & 15.405 & 2440 & 124 & 0.426 \\
\hline Total & & 573078 & 25351 & 100.000 \\
\hline
\end{tabular}

Figure S24. Chromatogram of $\left(R_{\mathrm{p}}\right)-\mathbf{3 d}$.

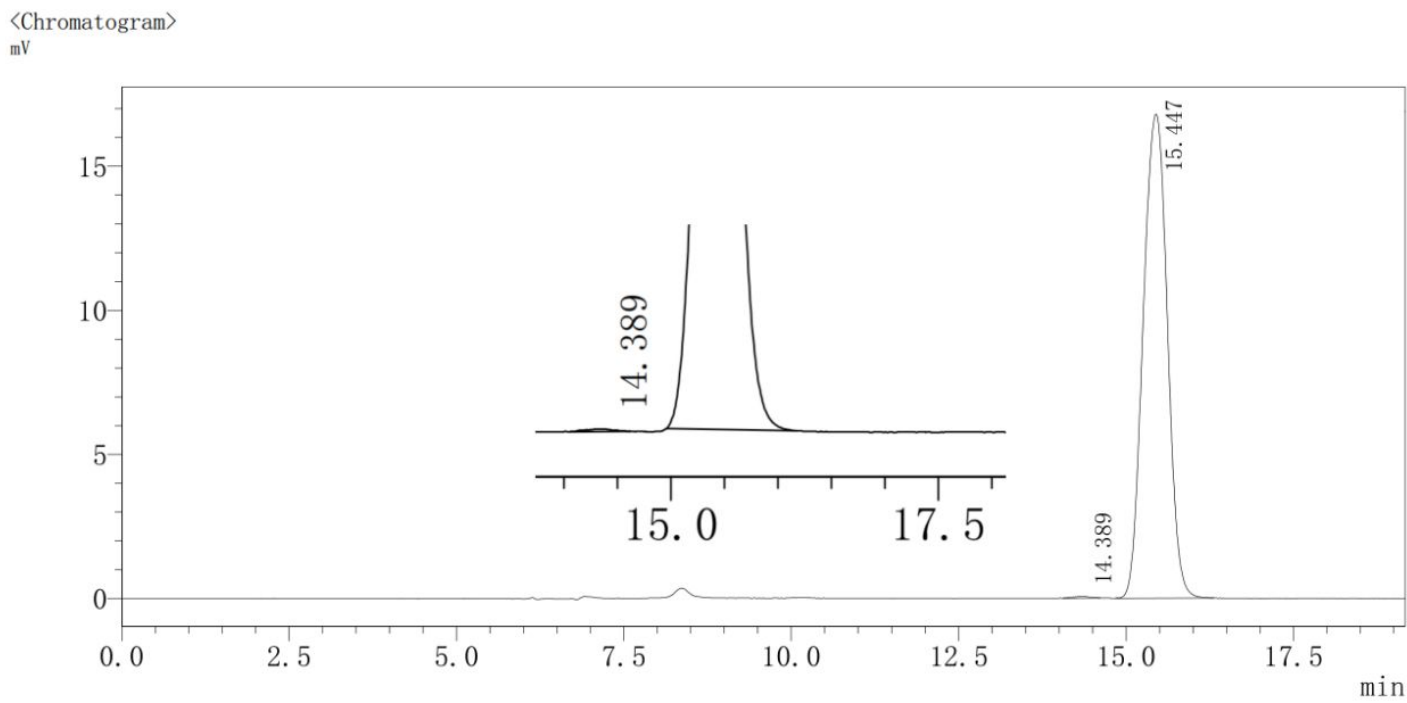

〈Peak Table〉

PDA Ch1 410nm

\begin{tabular}{|r|r|r|r|r|}
\hline Peak Name & Retention Time & Area & Peak Height & Area \% \\
\hline 1 & 14.389 & 1148 & 56 & 0.272 \\
\hline 2 & 15.447 & 420823 & 16790 & 99.728 \\
\hline Total & & 421971 & 16846 & 100.000 \\
\hline
\end{tabular}

Figure S25. Chromatogram of $\left(S_{\mathrm{p}}\right)$-3d. 
〈Chromatogram〉

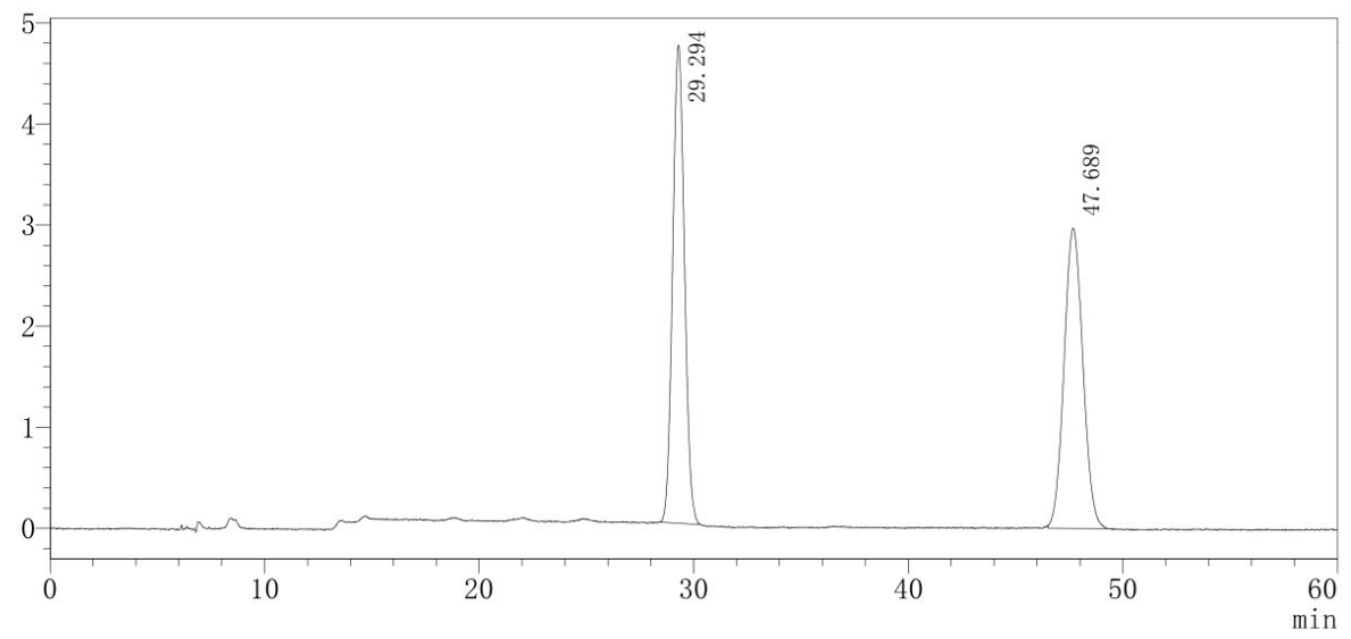

〈Peak Table〉

PDA Ch1 410nm

\begin{tabular}{|r|c|c|c|r|}
\hline Peak Name & Retention Time & Area & Peak Height & Area \% \\
\hline 1 & 29.294 & 180585 & 4728 & 50.060 \\
\hline 2 & 47.689 & 180152 & 2971 & 49.940 \\
\hline Total & & 360737 & 7699 & 100.000 \\
\hline
\end{tabular}

Figure S26. Chromatograms of the racemic of $\mathbf{3 e .}$

$\langle$ Chromatogram〉

$\mathrm{m} V$

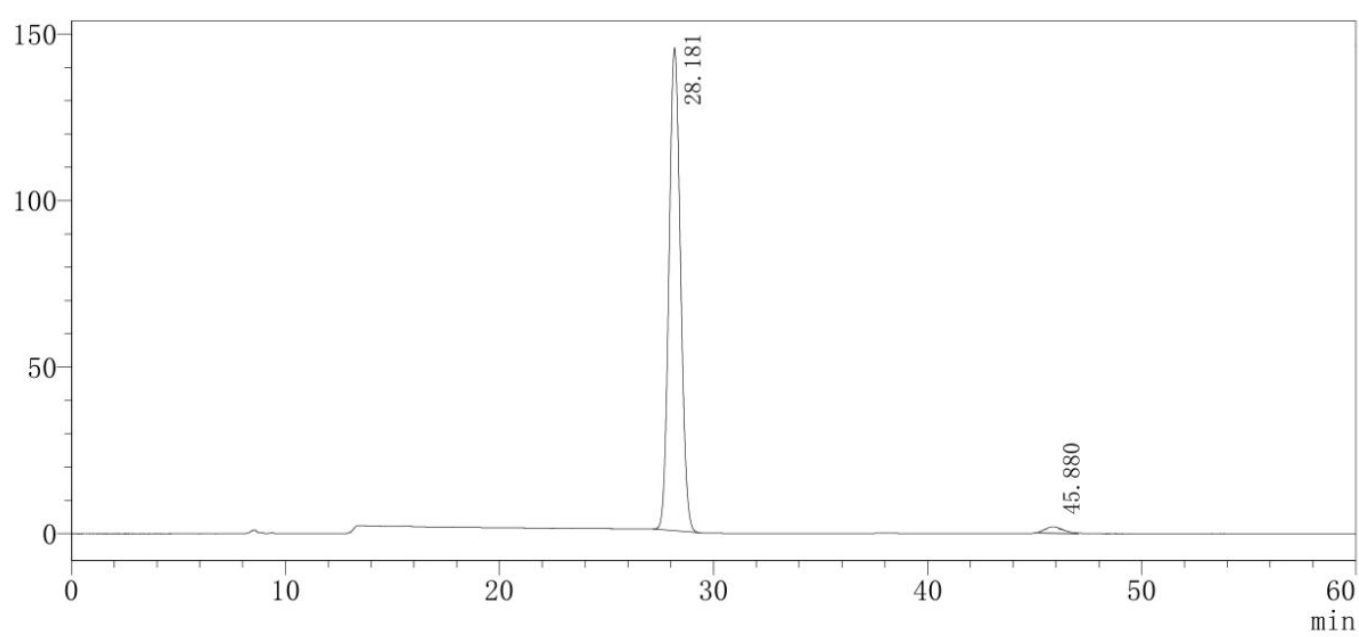

〈Peak Table〉

PDA Ch1 410nm

\begin{tabular}{|r|r|r|r|r|}
\hline Peak Name & Retention Time & Area & Peak Height & Area \% \\
\hline 1 & 28.181 & 5419255 & 144947 & 99.542 \\
\hline 2 & 45.880 & 24910 & 802 & 0.458 \\
\hline Total & & 5444165 & 145748 & 100.000 \\
\hline
\end{tabular}

Figure S27. Chromatogram of $\left(R_{\mathrm{p}}\right)$-3e. 


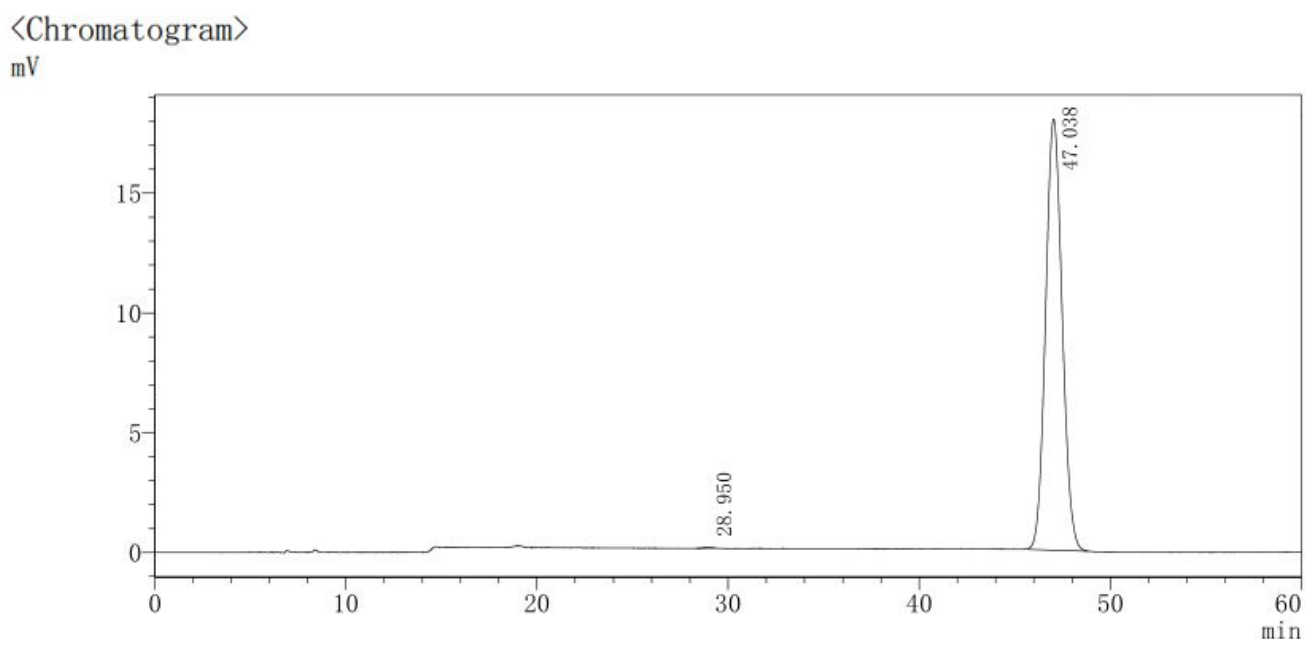

$\langle$ Peak Table $\rangle$

PDA Ch1 410nm

\begin{tabular}{|r|r|r|r|r|}
\hline Peak Name & Retention Time & Area & Peak Height & Area \% \\
\hline 1 & 28.950 & 696 & 29 & 0.064 \\
\hline 2 & 47.038 & 1084737 & 17997 & 99.936 \\
\hline Total & & 1085433 & 18026 & 100.000 \\
\hline
\end{tabular}

Figure S28. Chromatogram of $\left(S_{\mathrm{p}}\right)$-3e.

\section{General Procedure for crystal preparation and measurement.}

Compounds $\left(S_{p}\right)$-3a, $\left(R_{p}\right)$-3c, $\mathbf{3 d}$ and $\mathbf{3 e}$ (around $10 \mathrm{mg}$ ) were dissolved in $\mathrm{CH}_{2} \mathrm{Cl}_{2}(2 \mathrm{~mL})$ in a $5 \mathrm{~mL}$ vial, respectively. The single crystals were grown by slow evaporation of solvent at room temperature.

Intensity data of $\left(S_{p}\right)$-3a, 3d and 3e were collected on a Rigaku Oxford Diffraction Synergy Custom DW system X-ray diffractometer with a Hypix detector using $\mathrm{Cu}-\mathrm{K \alpha}$ radiation $(\lambda=1.54184 \AA)$ at $293 \mathrm{~K} ;\left(R_{p}\right)-3 \mathbf{c}$, was collected on a Gemini Eos X-ray diffractometer with $\mathrm{Cu}-\mathrm{K \alpha}$ radiation $(\lambda=1.54184 \AA)$ at $100 \mathrm{~K}$. The structure was solved by direct methods and refined by full-matrix least-squares methods with SHELX-2014 program. ${ }^{3}$ Displacement parameters were refined anisotropically, and the positions of the H-atoms were generated geometrically, assigned isotropic thermal parameters, and allowed to ride on their parent carbon atoms before the final cycle of refinement. Basic information pertaining to crystal parameters and structure refinement are summarized in Table S1, and hydrogen bonds are listed in Table S2. CCDC $2090499\left(S_{p}-\mathbf{3 a}\right)$, $2090500\left(R_{p}-\mathbf{3 c}\right), 2090501(\mathbf{3 d}), 2090502(\mathbf{3 e})$ contain the supplementary crystallographic data for this paper. 


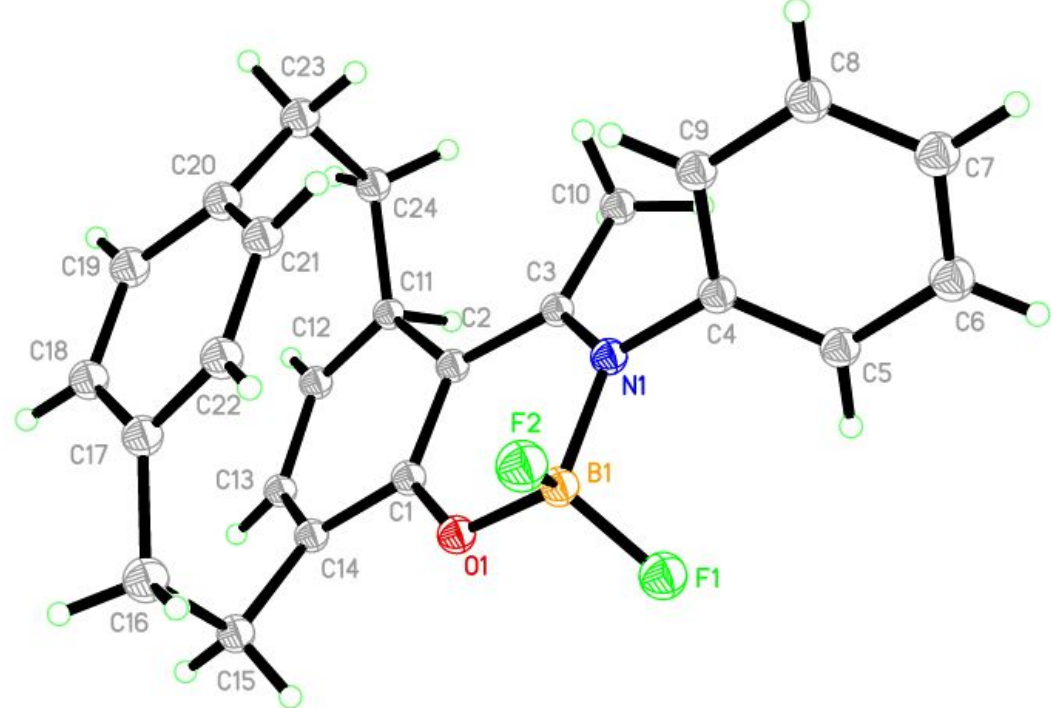

Figure S29. Single crystals of compound $\left(S_{p}\right)$-3a (Ellipsoids are drawn to $30 \%$ probability).

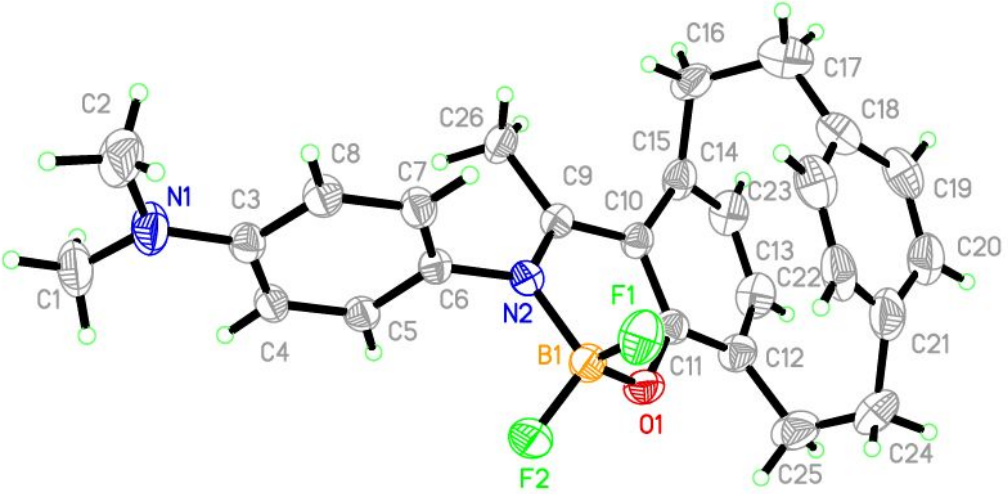

Figure S30. Single crystals of compound $\left(R_{p}\right)$-3c (Ellipsoids are drawn to $30 \%$ probability). 


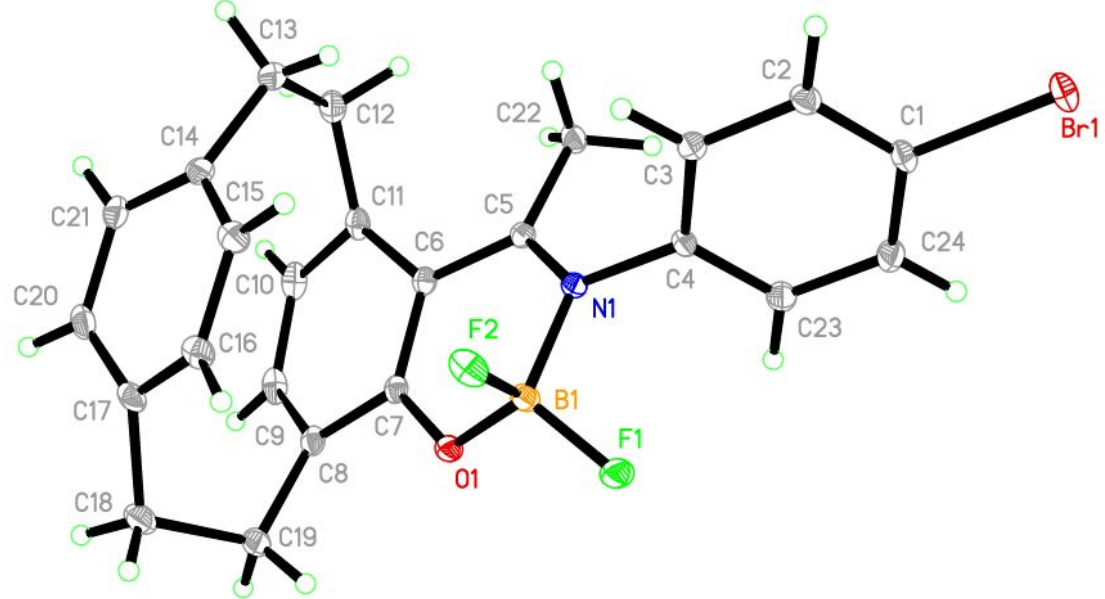

Figure S31. Single crystals of compound 3d (Ellipsoids are drawn to 30\% probability).

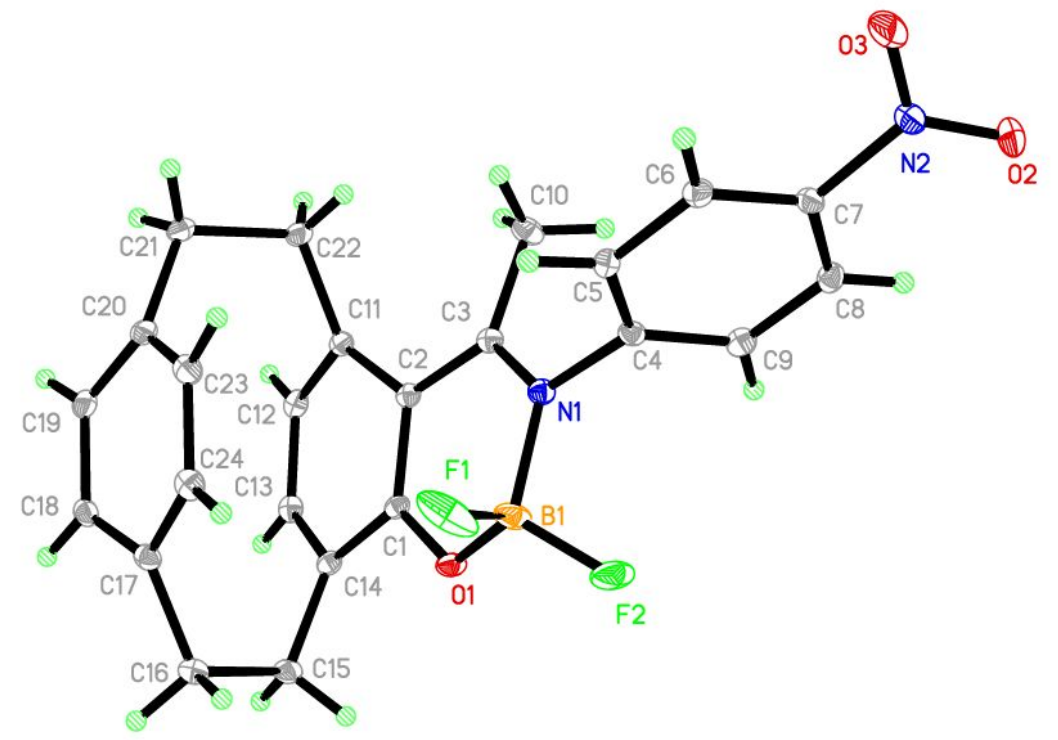

Figure S32. Single crystals of compound 3e (Ellipsoids are drawn to 30\% probability). 


\section{X-ray crystallographic analysis}

Table S9. Crystallographic data and structure refinement for $\left(S_{p}\right)-\mathbf{3 a},\left(R_{p}\right)-\mathbf{3 c}, \mathbf{3 d}$ and $\mathbf{3 e}$.

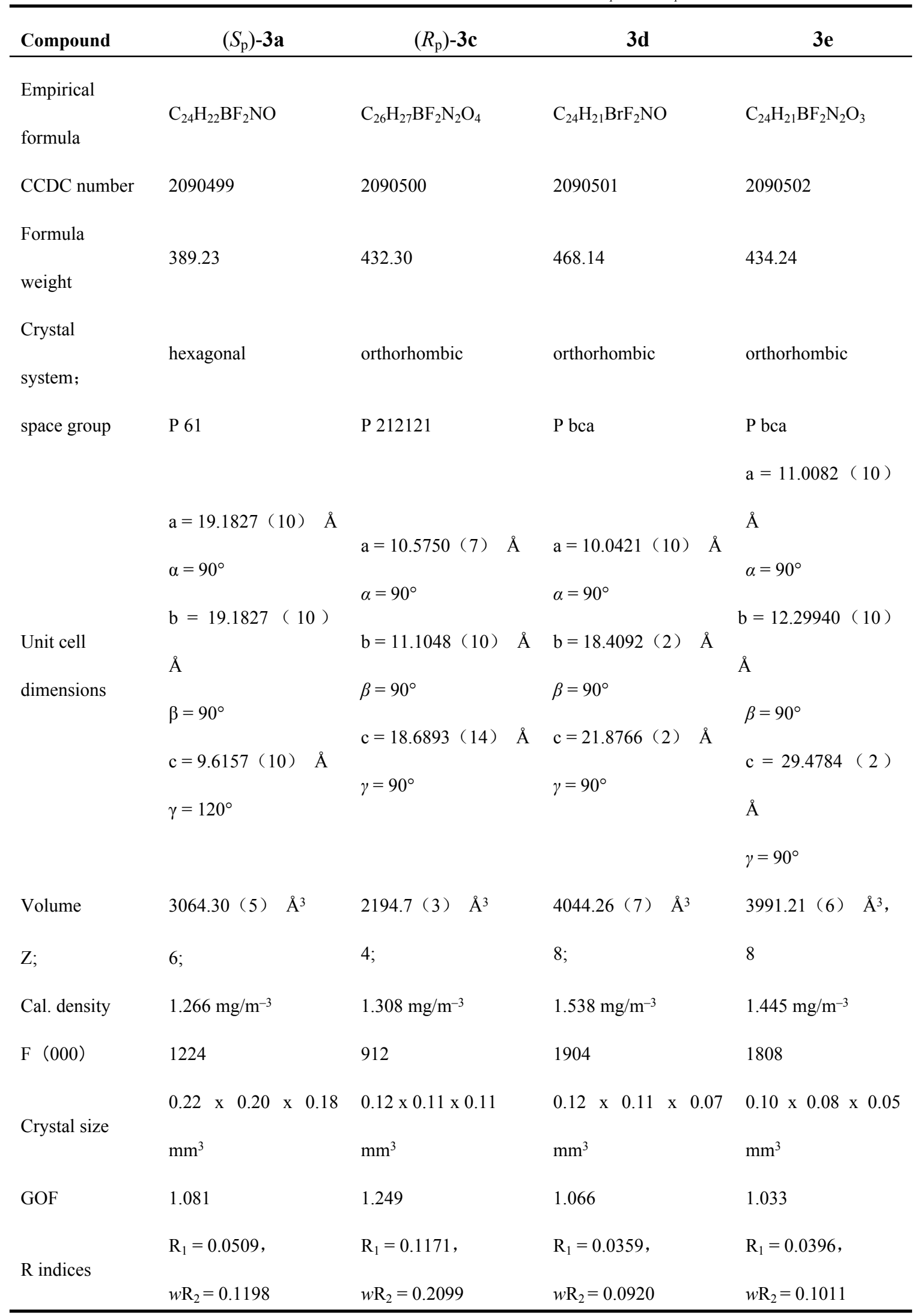


Table S10. Hydrogen bonds for $\left(S_{p}\right)-3 \mathbf{a},\left(R_{p}\right)-\mathbf{3 c}, \mathbf{3 d}$ and $\mathbf{3 e}\left[\AA\right.$ and $\left.{ }^{\circ}\right]$

\begin{tabular}{cccc}
\hline Compound & $\mathrm{C}-\mathrm{H} \cdots \mathrm{A}$ & $\mathrm{D}(\mathrm{H} \cdots \mathrm{A})$ & $\angle(\mathrm{DHA})$ \\
\hline$\left(S_{p}\right)$-3a & $\mathrm{C} 21-\mathrm{H} 21 \mathrm{~A} \cdots \mathrm{B} 1$ & $3.11 \AA$ & $165.2^{\circ}$ \\
& $\mathrm{C} 9-\mathrm{H} 9 \cdots \mathrm{F} 2$ & $2.45 \AA$ & $149.9^{\circ}$ \\
& $\mathrm{C} 10-\mathrm{H} 10 \mathrm{~A} \cdots \mathrm{F} 1$ & $2.29 \AA$ & $161.6^{\circ}$ \\
& $\mathrm{C} 13-\mathrm{H} 13 \cdots \pi$ & $2.49 \AA$ & $164.5^{\circ}$ \\
$\left(R_{p}\right)-\mathbf{3 c}$ & $\mathrm{C} 8-\mathrm{H} 8 \cdots \mathrm{F} 2$ & $2.60 \AA$ & $138.3^{\circ}$ \\
& $\mathrm{C} 17-\mathrm{H} 17 \mathrm{~A} \cdots \mathrm{F} 2$ & $2.50 \AA$ & \\
& $\mathrm{C} 19-\mathrm{H} 19 \cdots \pi$ & $2.84 \AA$ & $156.1^{\circ}$ \\
3d & $\mathrm{C} 2-\mathrm{H} 2 \cdots \mathrm{F} 2$ & $2.60 \AA$ & $166.5^{\circ}$ \\
& $\mathrm{C} 10-\mathrm{H} 10 \cdots \mathrm{F} 1$ & $2.30 \AA$ & $140.7^{\circ}$ \\
& $\mathrm{C} 23-\mathrm{H} 23 \cdots \mathrm{F} 1$ & $2.59 \AA$ & $129.6^{\circ}$ \\
& $\mathrm{C} 24-\mathrm{H} 24 \cdots \mathrm{O} 1$ & $2.65 \AA$ & $124.8^{\circ}$ \\
& $\mathrm{C} 12-\mathrm{H} 12 \mathrm{~B} \cdots \mathrm{Br} 1$ & $2.91 \AA$ & $156.7^{\circ}$ \\
& $\mathrm{C} 13-\mathrm{H} 13 \mathrm{~B} \cdots \mathrm{Br} 1$ & $2.87 \AA$ & $83.9^{\circ}$ \\
& $\mathrm{C} 8-\mathrm{H} 8 \cdots \mathrm{F} 1$ & $2.94 \AA$ & $127.7^{\circ}$ \\
& $\mathrm{C} 6-\mathrm{H} 6 \cdots \mathrm{F} 2$ & $2.56 \AA$ & $146.8^{\circ}$ \\
& $\mathrm{C} 9-\mathrm{H} 9 \cdots \mathrm{O} 1$ & $2.67 \AA$ & $116.1^{\circ}$ \\
& $\mathrm{C} 19-\mathrm{H} 19 \cdots \mathrm{O} 2$ & $2.96 \AA$ & $161.0^{\circ}$ \\
& $\mathrm{C} 16-\mathrm{H} 16 \mathrm{~B} \cdots \mathrm{O} 2$ & $2.72 \AA$ & $147.6^{\circ}$ \\
& $\mathrm{C} 22-\mathrm{H} 22 \mathrm{~A} \cdots \mathrm{O} 2$ & $2.71 \AA$ & $147.3^{\circ}$ \\
& $\mathrm{C} 10-\mathrm{H} 10 \mathrm{~N} 2 \mathrm{O} 3$ & $2.43 \AA$ & \\
& $\mathrm{C} 10-\mathrm{H} 10 \mathrm{~B} \cdots \pi$ & $2.61 \AA$ & \\
& & &
\end{tabular}

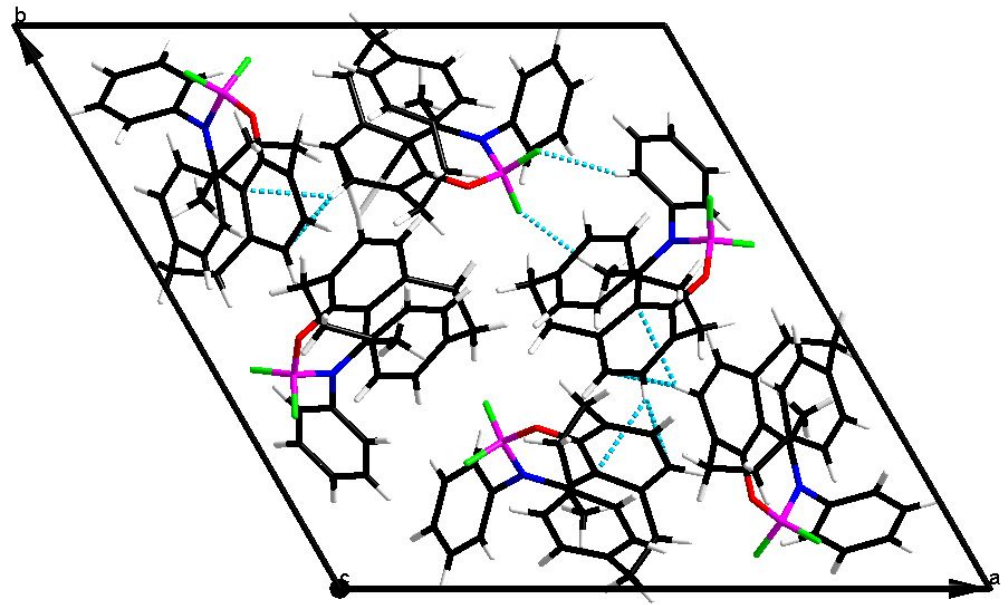

Figure S33. Molecular packing structure of $\left(S_{p}\right)$-3a along $c$ axis. The cyan dotted lines show weak intermolecular interactions. 


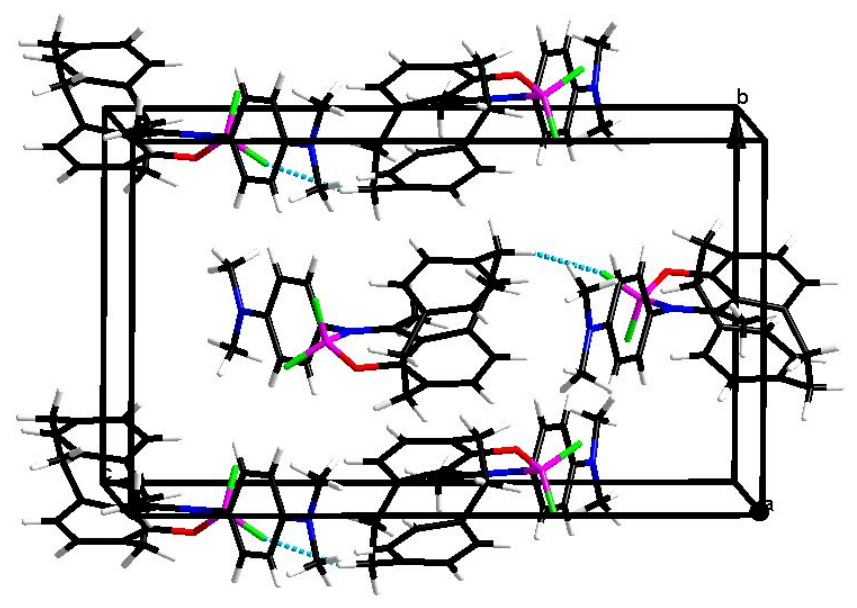

Figure S34. Molecular packing structure of $\left(R_{p}\right)$-3c along a axis. The cyan dotted lines show weak intermolecular interactions.

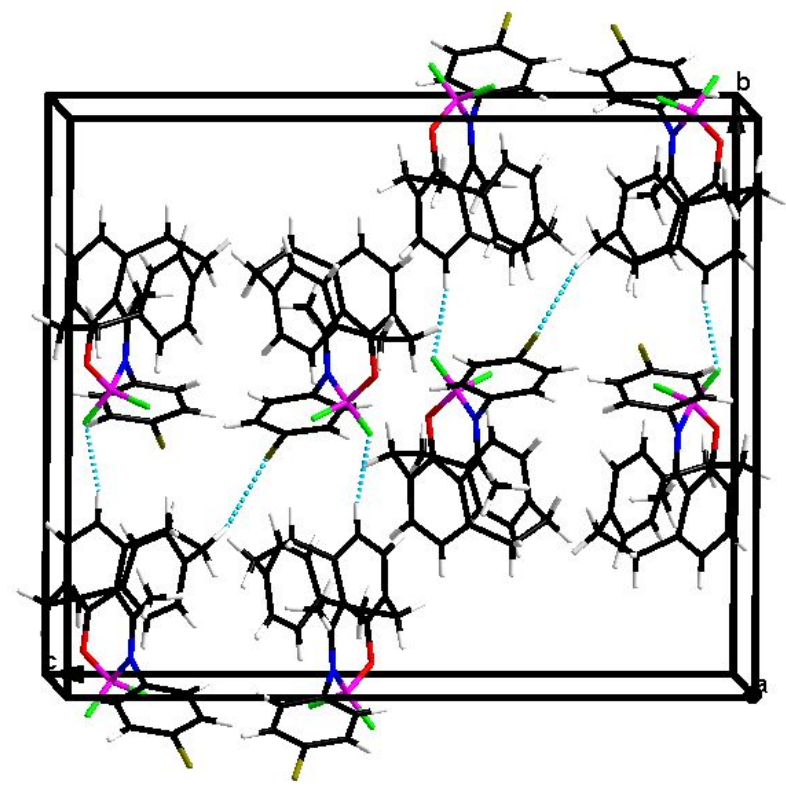

Figure S35. Molecular packing structure of 3d along $a$ axis. The cyan dotted lines show weak intermolecular interactions.

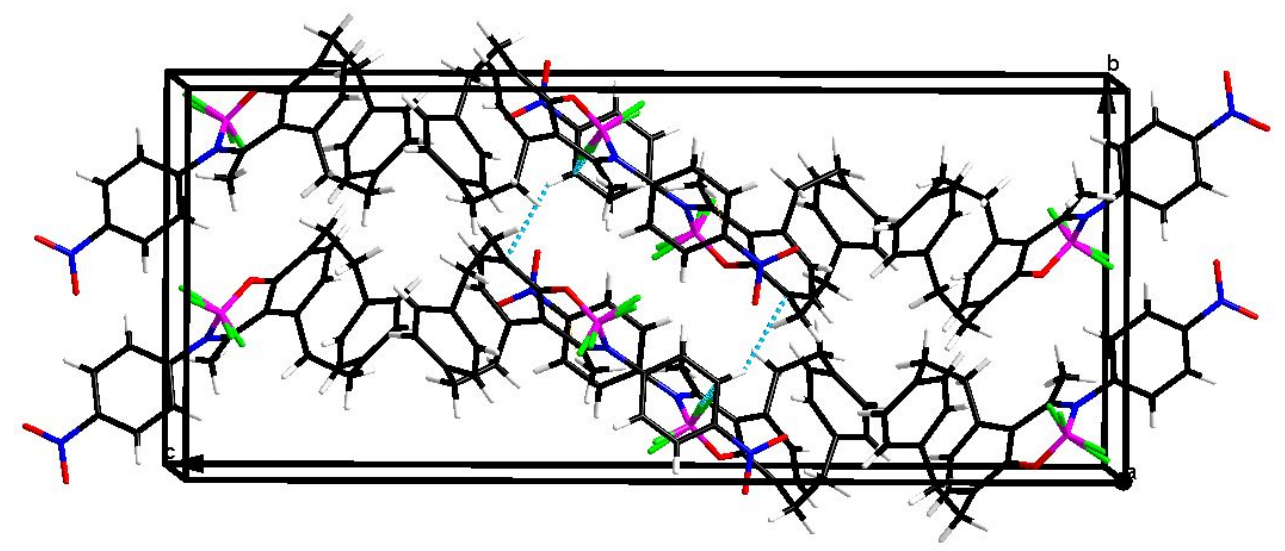

Figure S36. Molecular packing structure of 3e along $a$ axis. The cyan dotted lines show weak intermolecular interactions. 


\section{Copies of NMR and HRMS Spectra}

\section{$3.1{ }^{1} \mathrm{H}$ NMR and ${ }^{13} \mathrm{C}\left\{{ }^{1} \mathrm{H}\right\}$ NMR:}

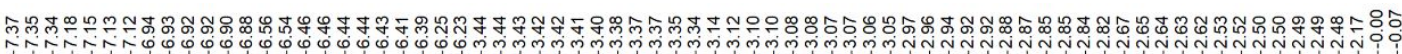<smiles></smiles>

$2 a$

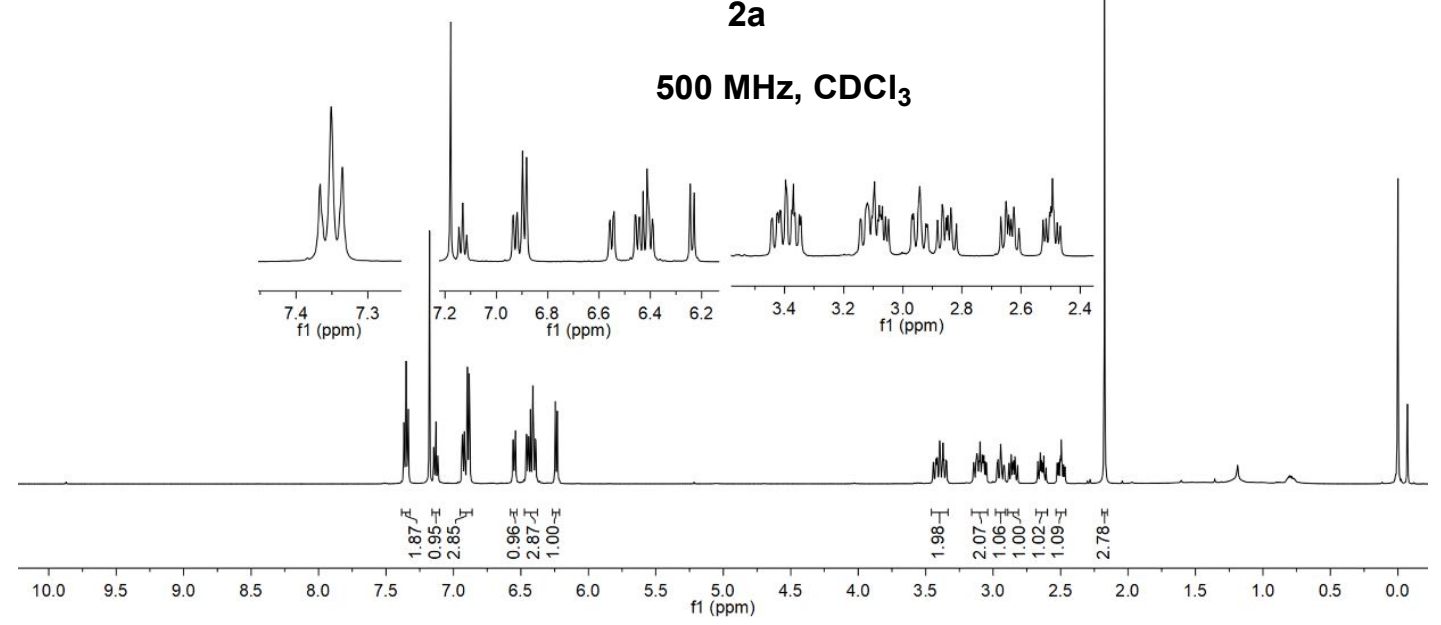

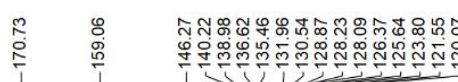<smiles>CC(=Nc1ccccc1)c1c(O)c2c(c3c1CC3)CC2</smiles>

$2 a$

$126 \mathrm{MHz}^{\mathrm{CDCl}_{3}}$

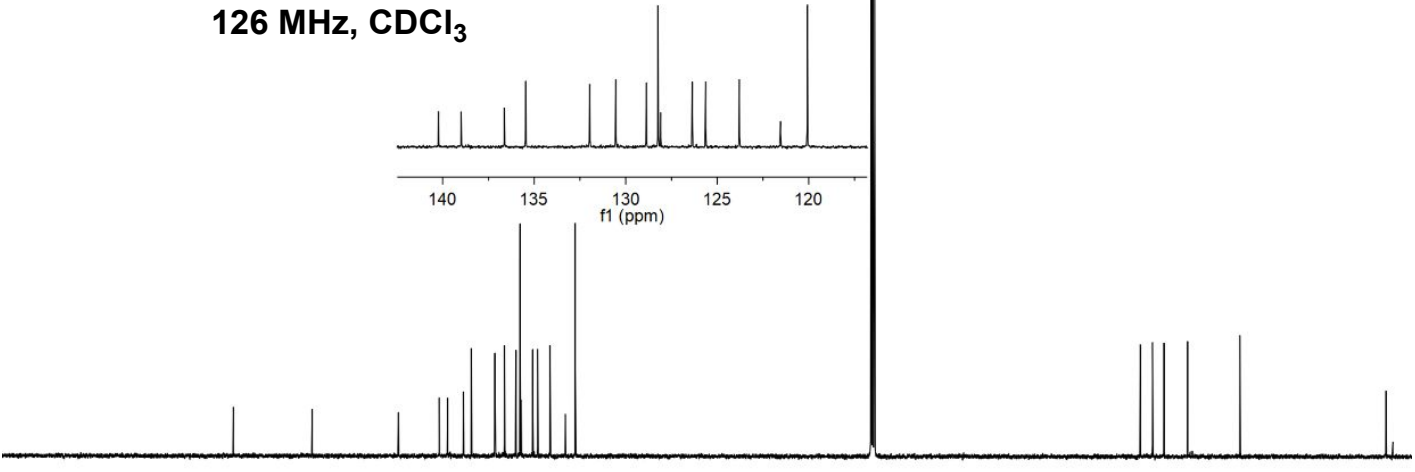

$\begin{array}{llllllllllllllllllllll}200 & 190 & 180 & 170 & 160 & 150 & 140 & 130 & 120 & 110 & 100 & 100 & 80 & 70 & 60 & 50 & 40 & 30 & 20 & 10 & 0\end{array}$ 
<smiles>COc1ccc(/N=C(\C)c2c3c(cc4c2CC4)CC3)cc1</smiles>

$400 \mathrm{MHz}, \mathrm{CDCl}_{3}$
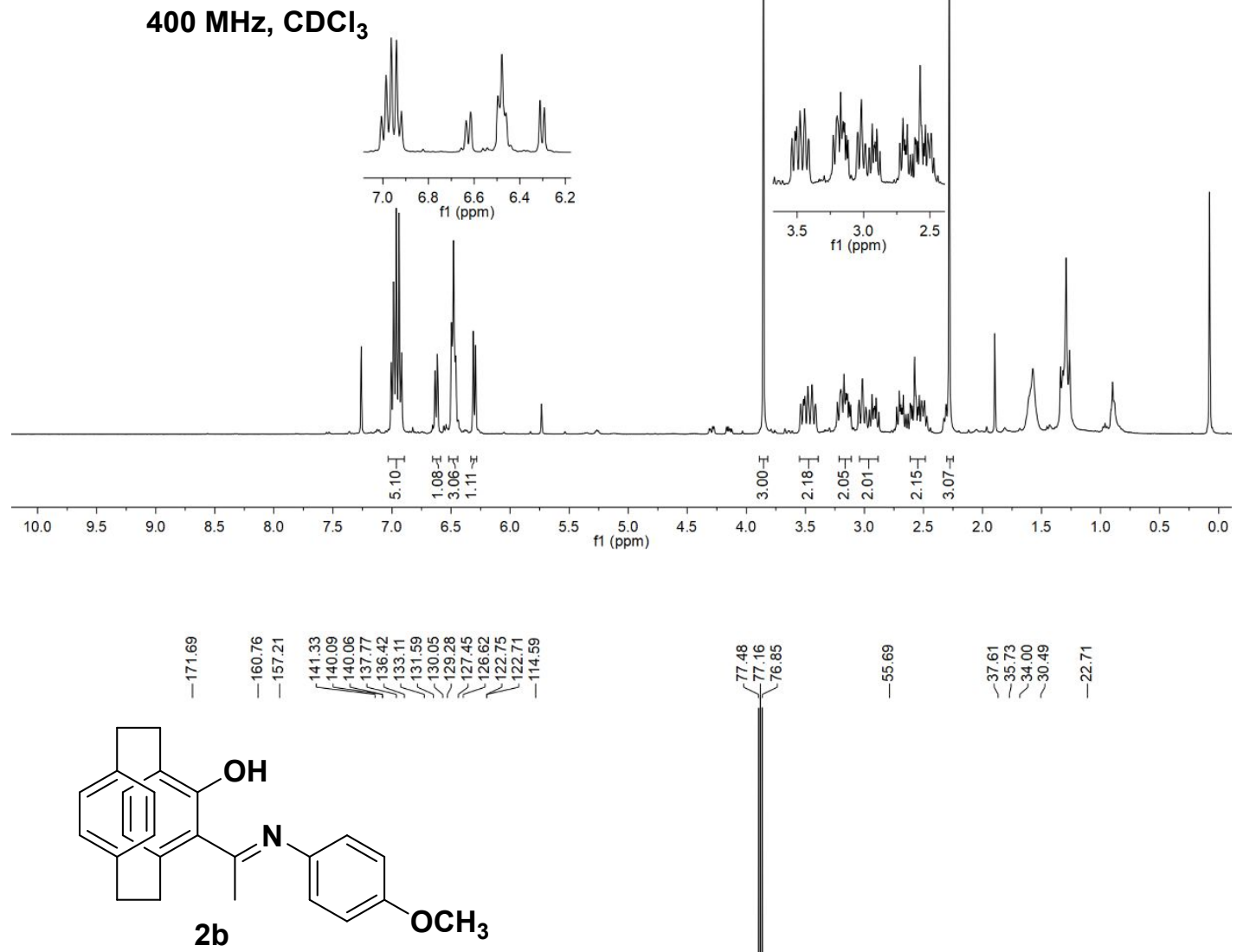

$101 \mathrm{MHz}, \mathrm{CDCl}_{3}$

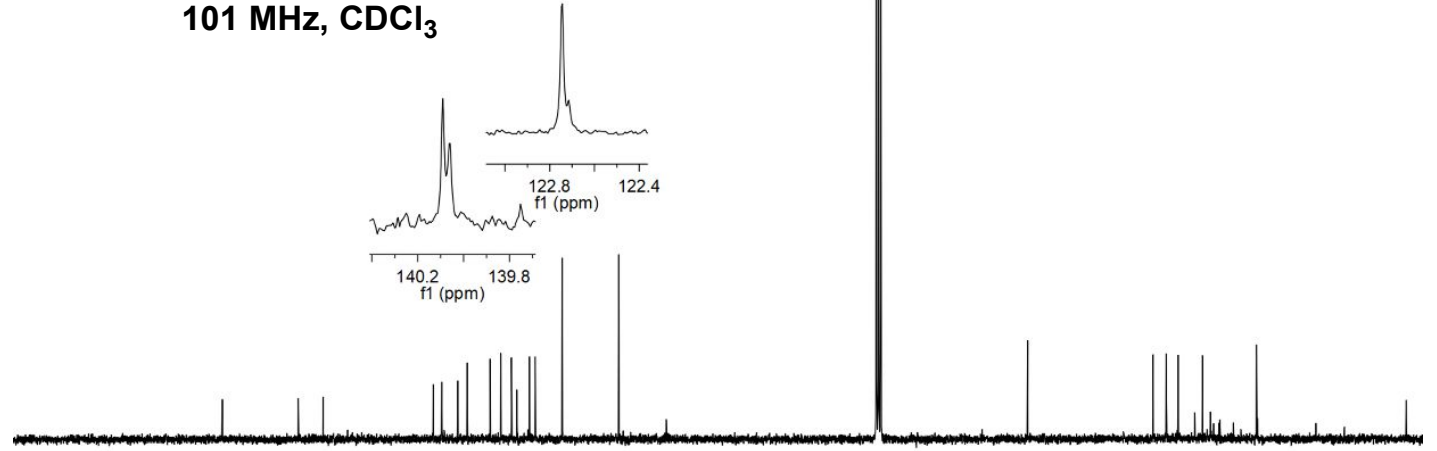

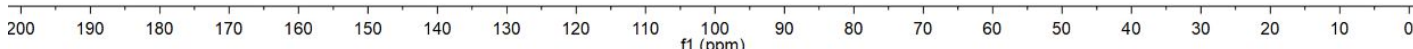




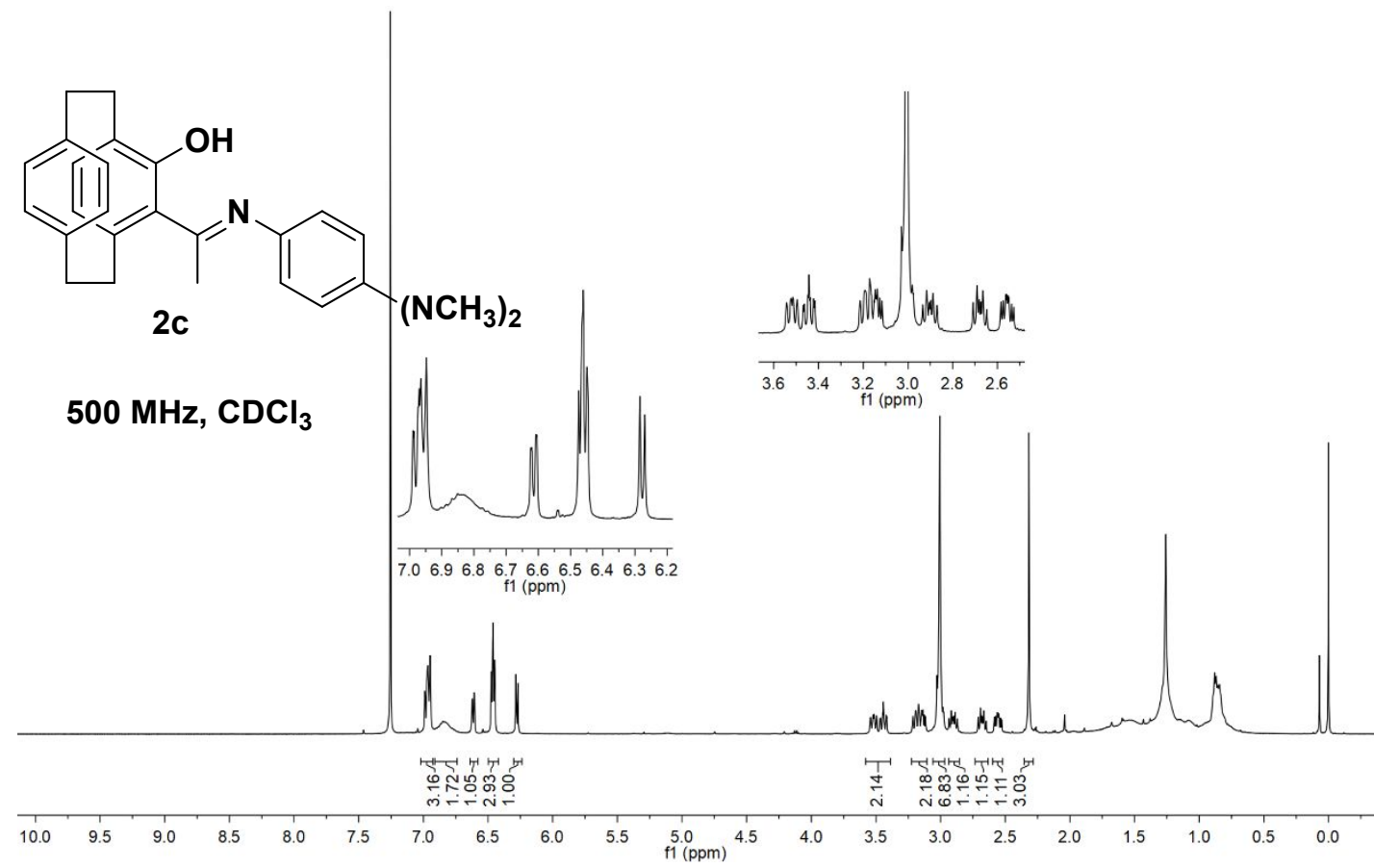

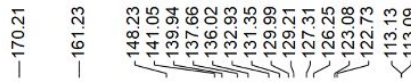

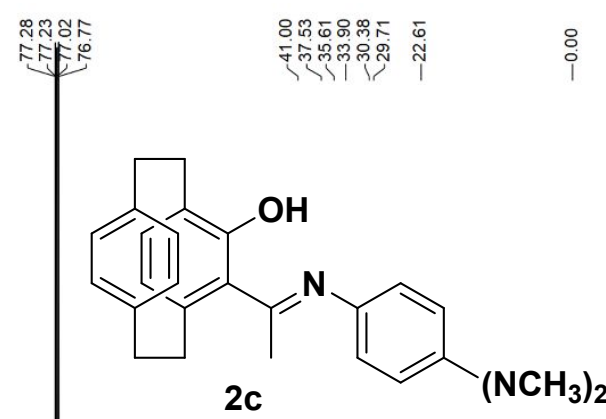

$126 \mathrm{MHz}^{\mathrm{CDCl}_{3}}$

$\begin{array}{lllllllllllllllllllllllllllll}1 & 190 & 180 & 170 & 160 & 150 & 140 & 130 & 120 & 110 & 100 & 90 & 80 & 70 & 60 & 50 & 40 & 30 & 20 & 10 & 0\end{array}$ 


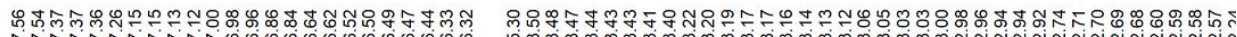

000000000 U

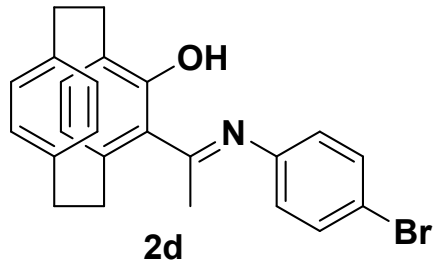

$400 \mathrm{MHz}, \mathrm{CDCl}_{3}$

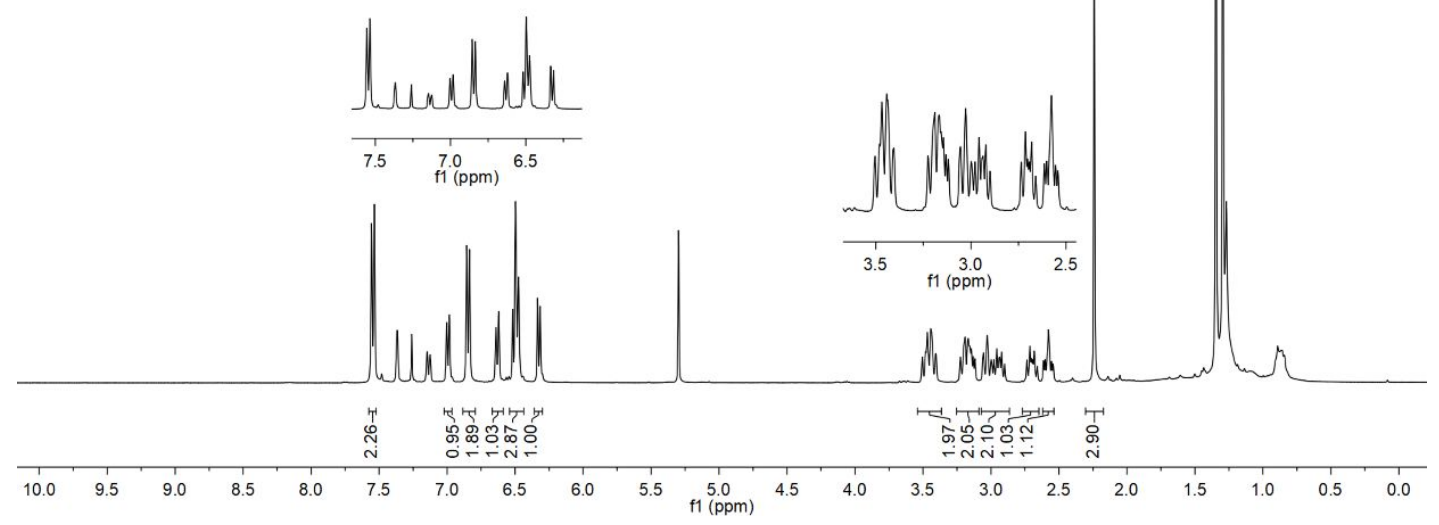

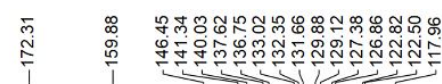

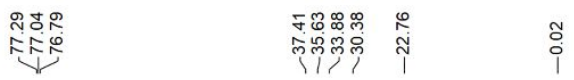

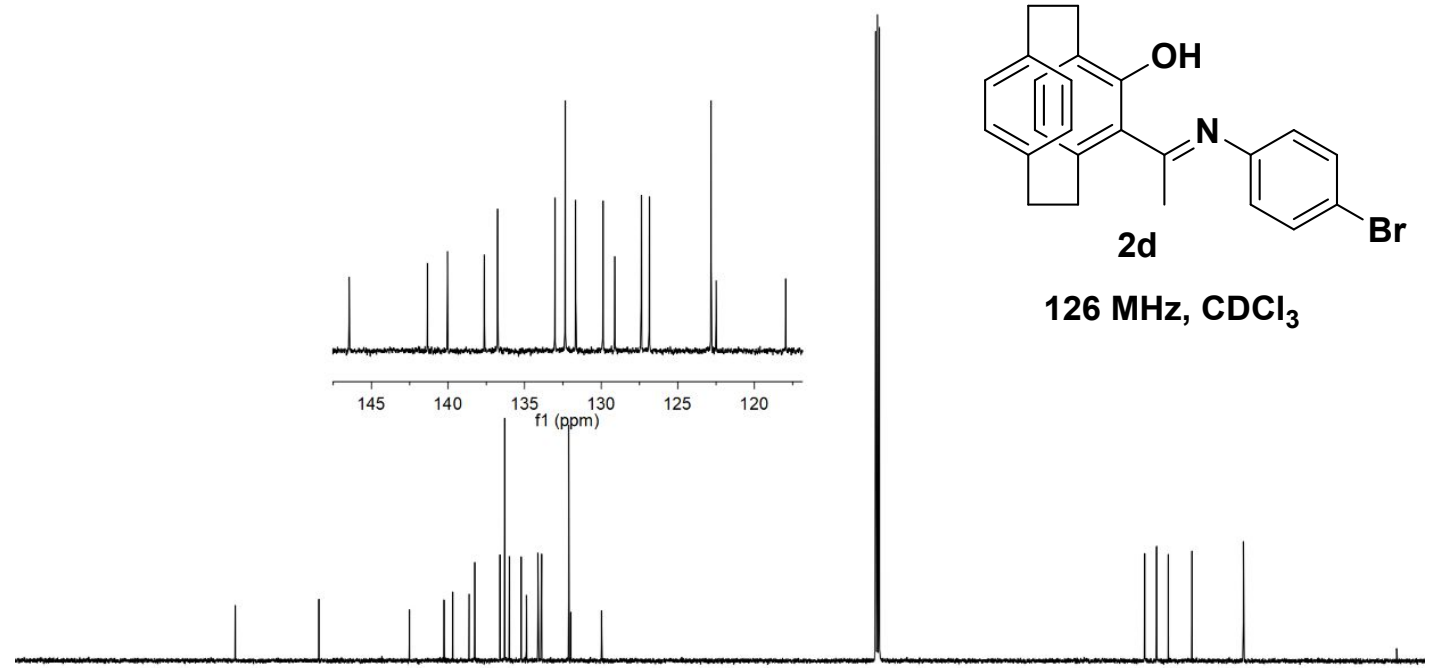

$126 \mathrm{MHz}, \mathrm{CDCl}_{3}$

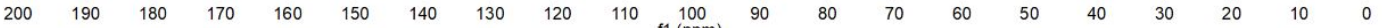



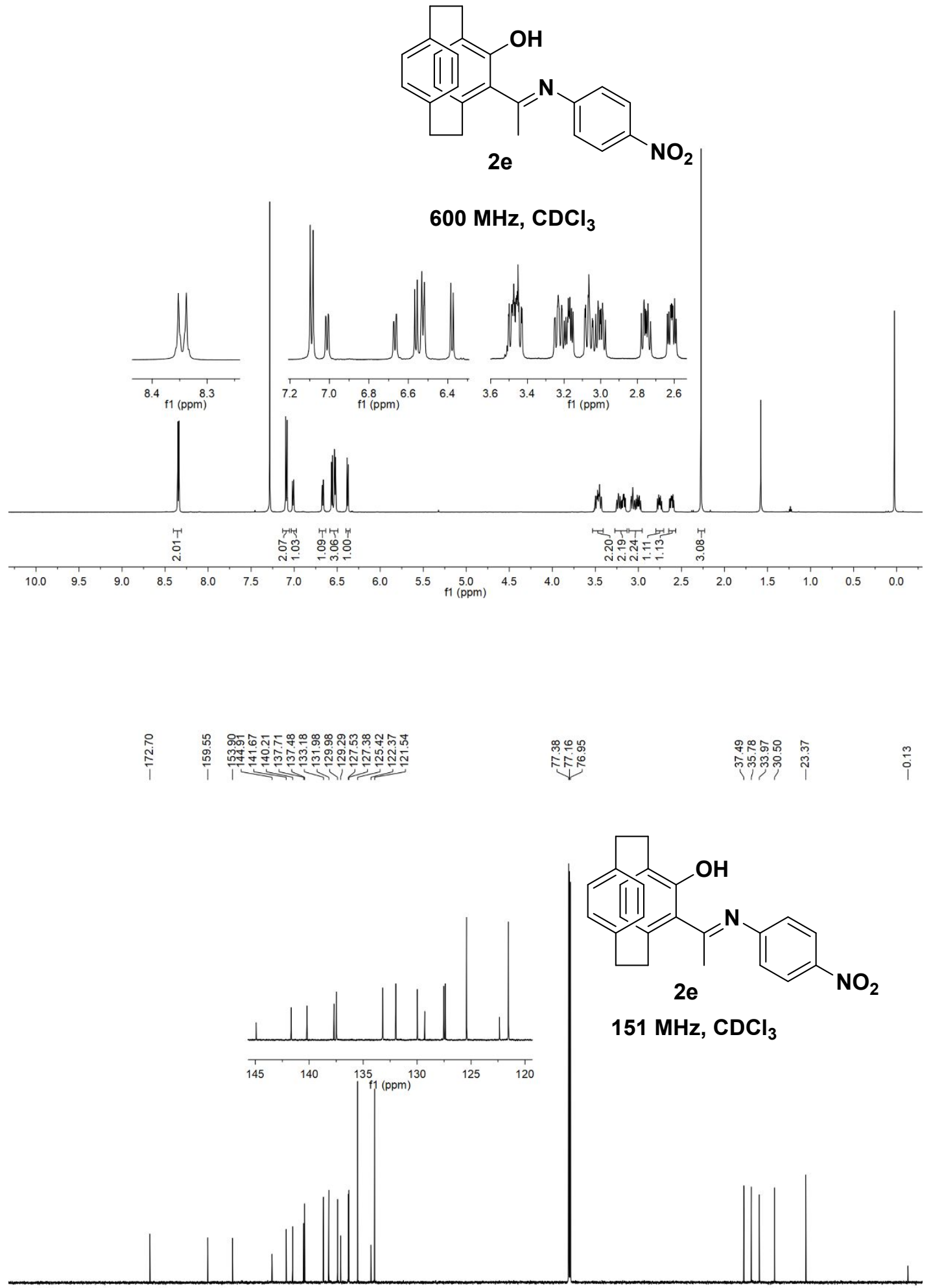

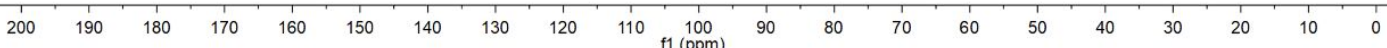




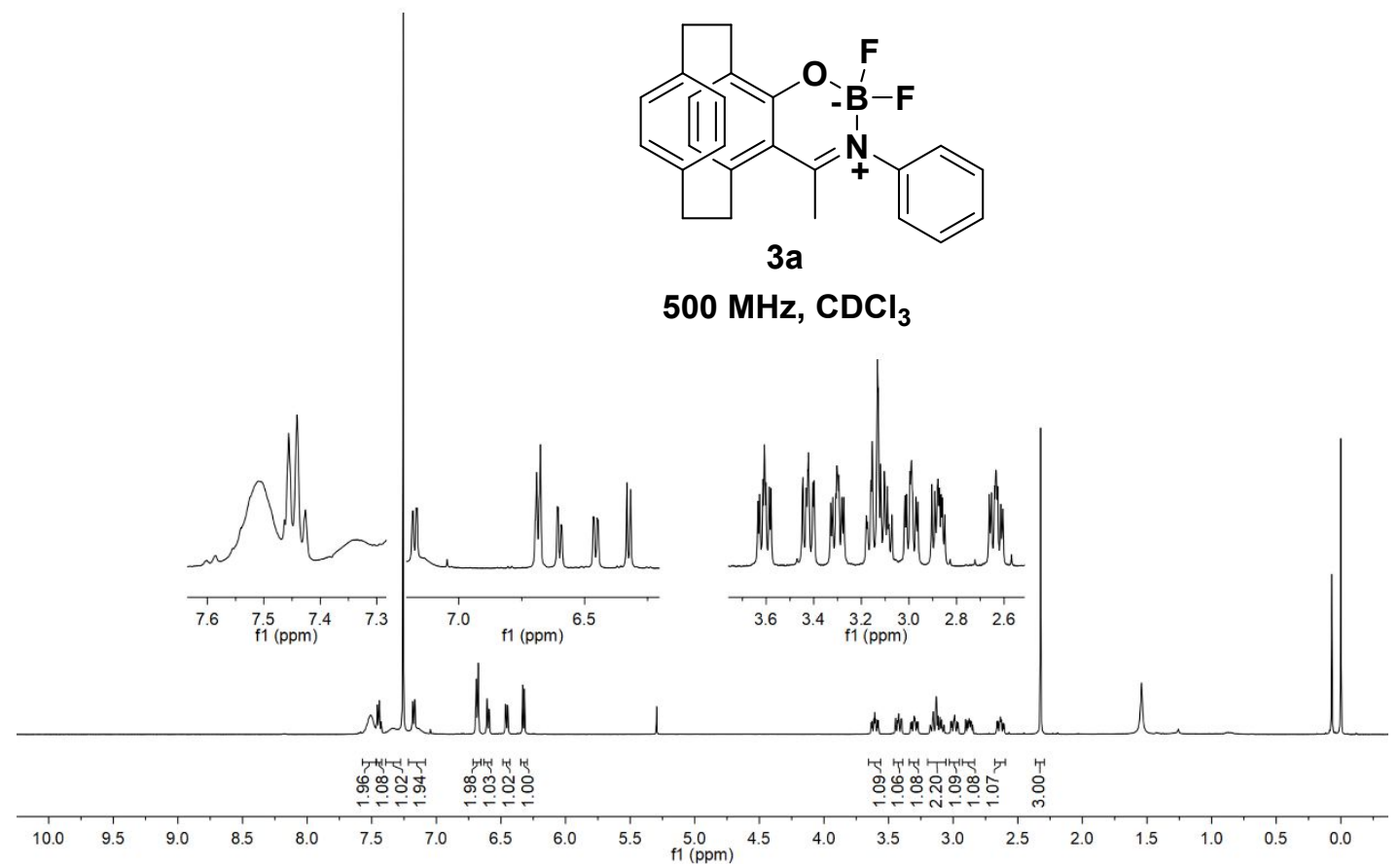

o :

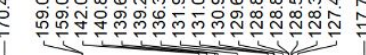

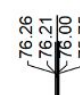

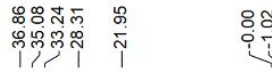

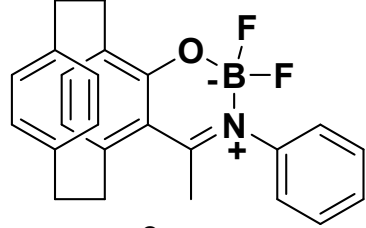

$3 a$

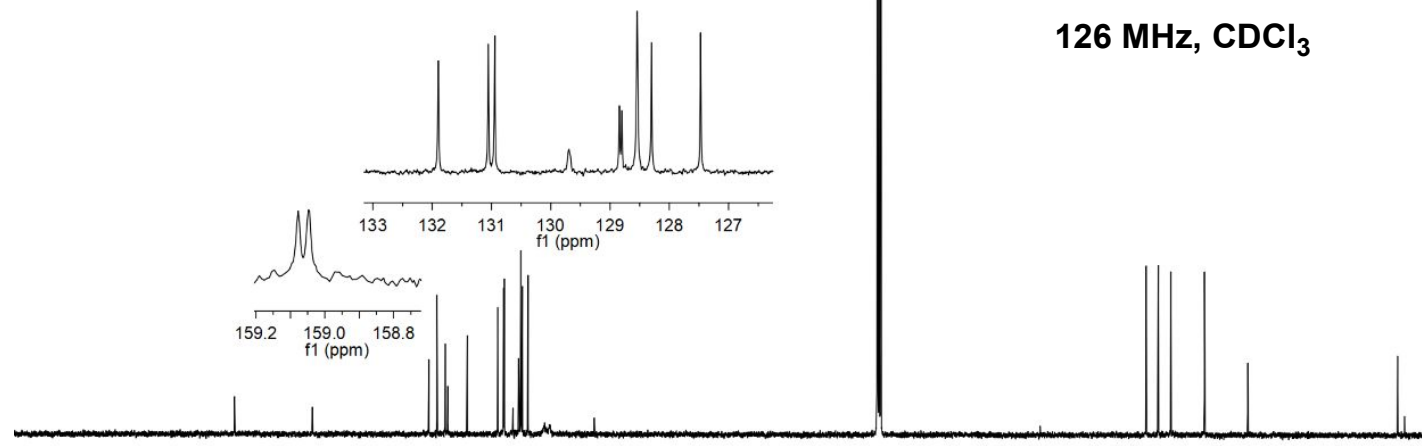

$\begin{array}{llllllllllllllllllllllll}200 & 190 & 180 & 170 & 160 & 150 & 140 & 130 & 120 & 110 & 100 & 90 & 80 & 70 & 60 & 50 & 40 & 30 & 20 & 10 & 0\end{array}$ 


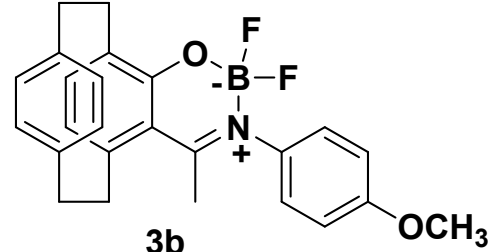

$500 \mathrm{MHz}, \mathrm{CDCl}_{3}$

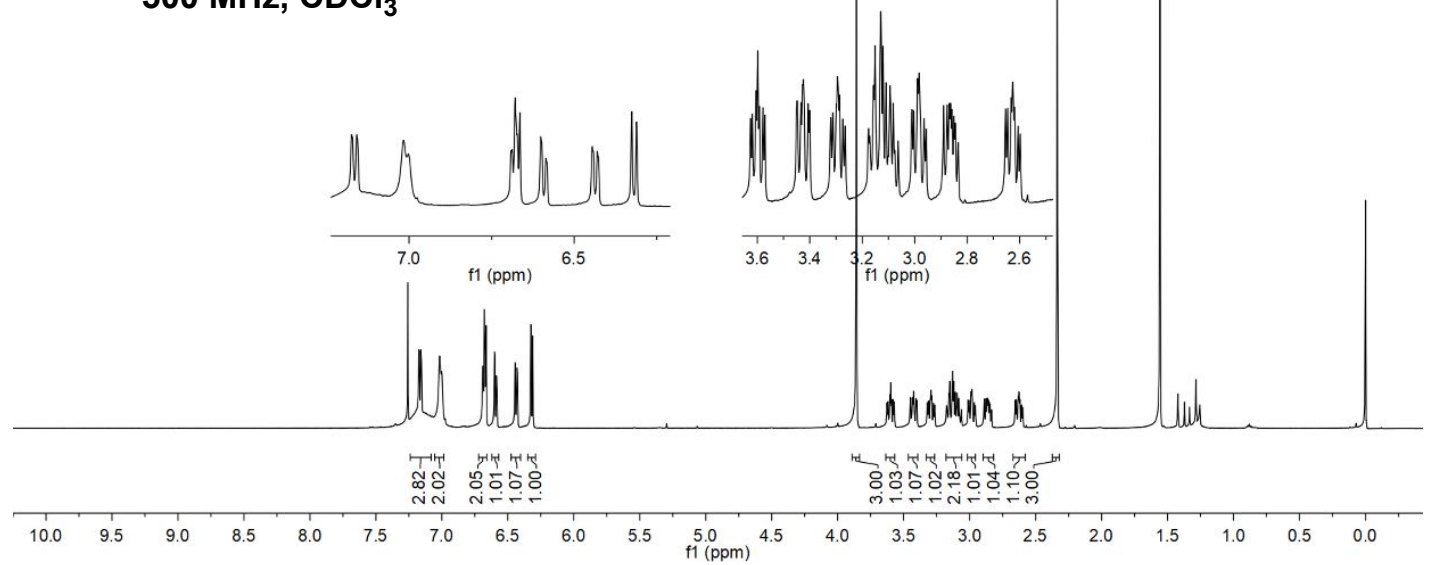

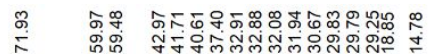

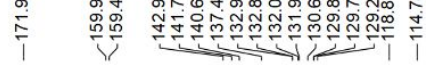

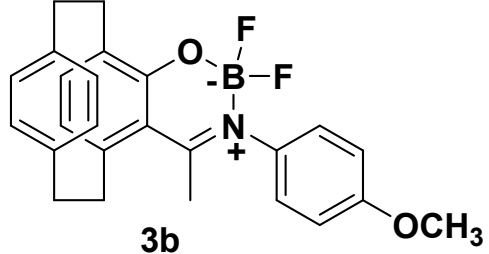

$3 b$

$126 \mathrm{MHz}, \mathrm{CDCl}_{3}$
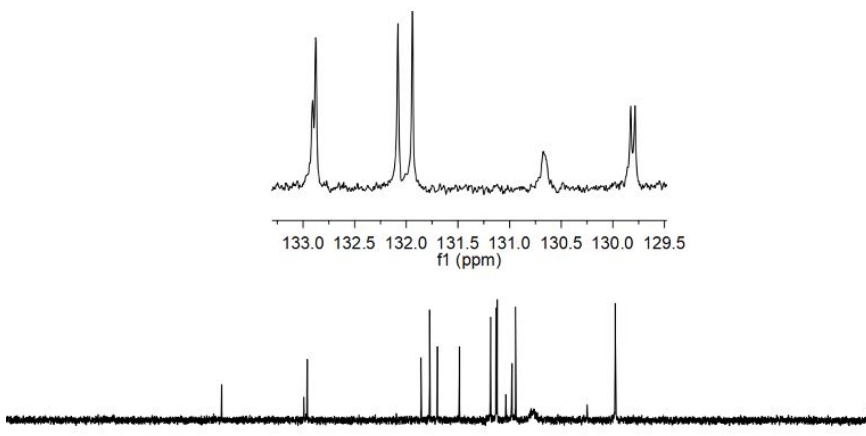

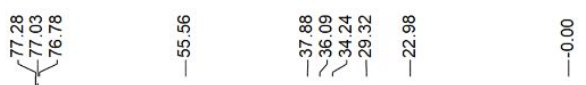

200

180

160

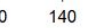

120

$110 \begin{gathered}100 \\ \mathrm{f} 1(\mathrm{ppm})\end{gathered}$ 


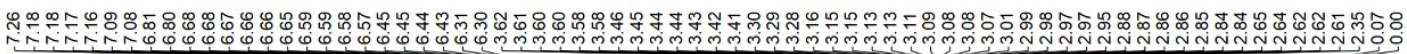

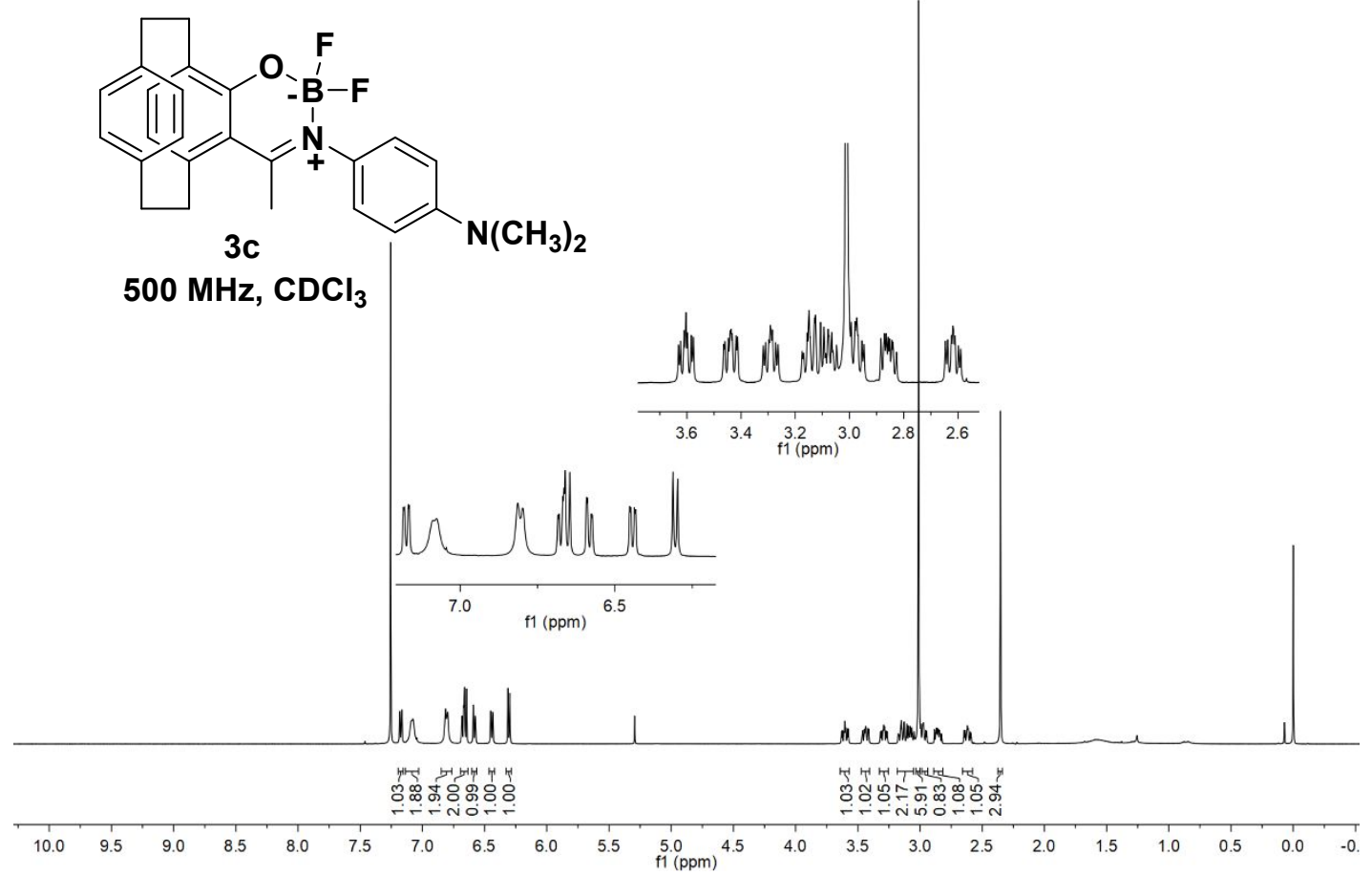

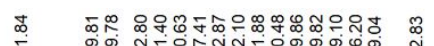

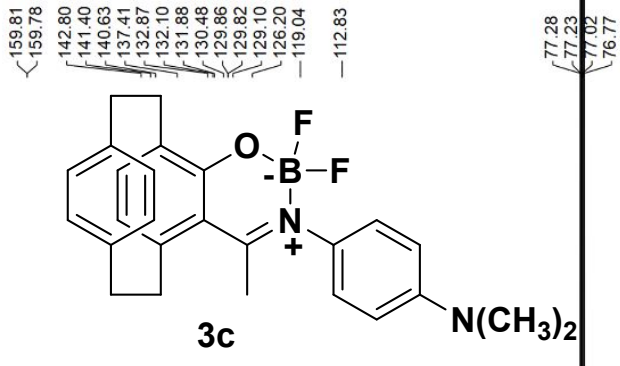

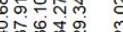

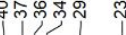

$126 \mathrm{MHz}, \mathrm{CDCl}_{3}$
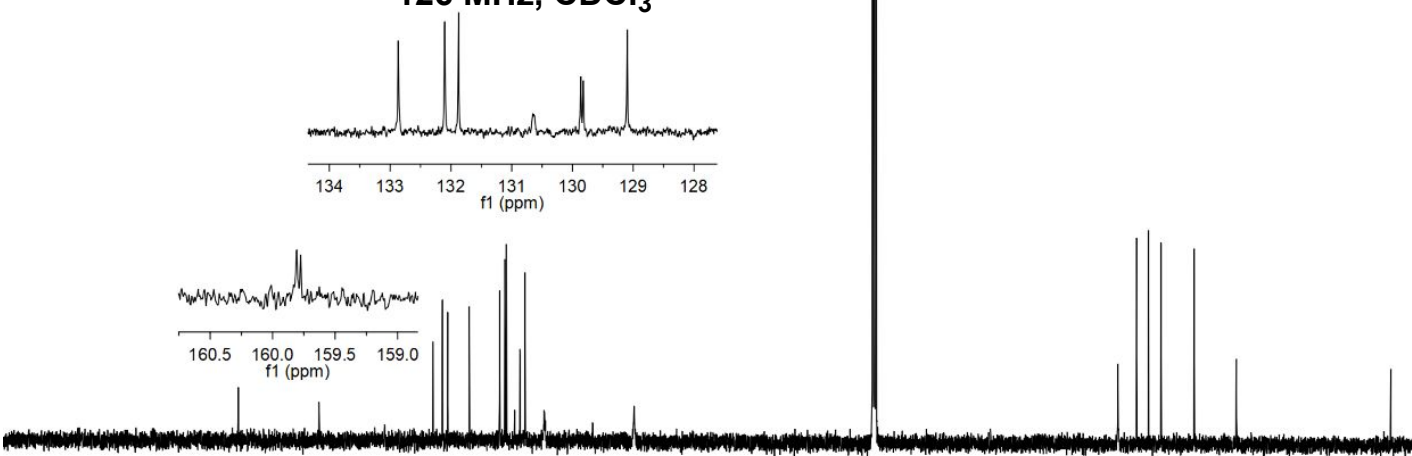

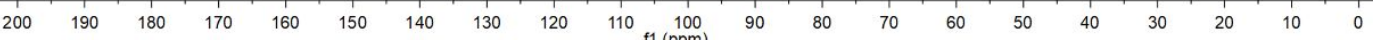




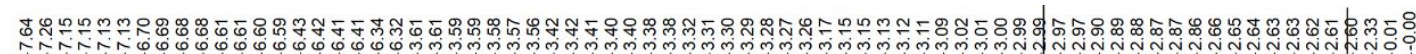

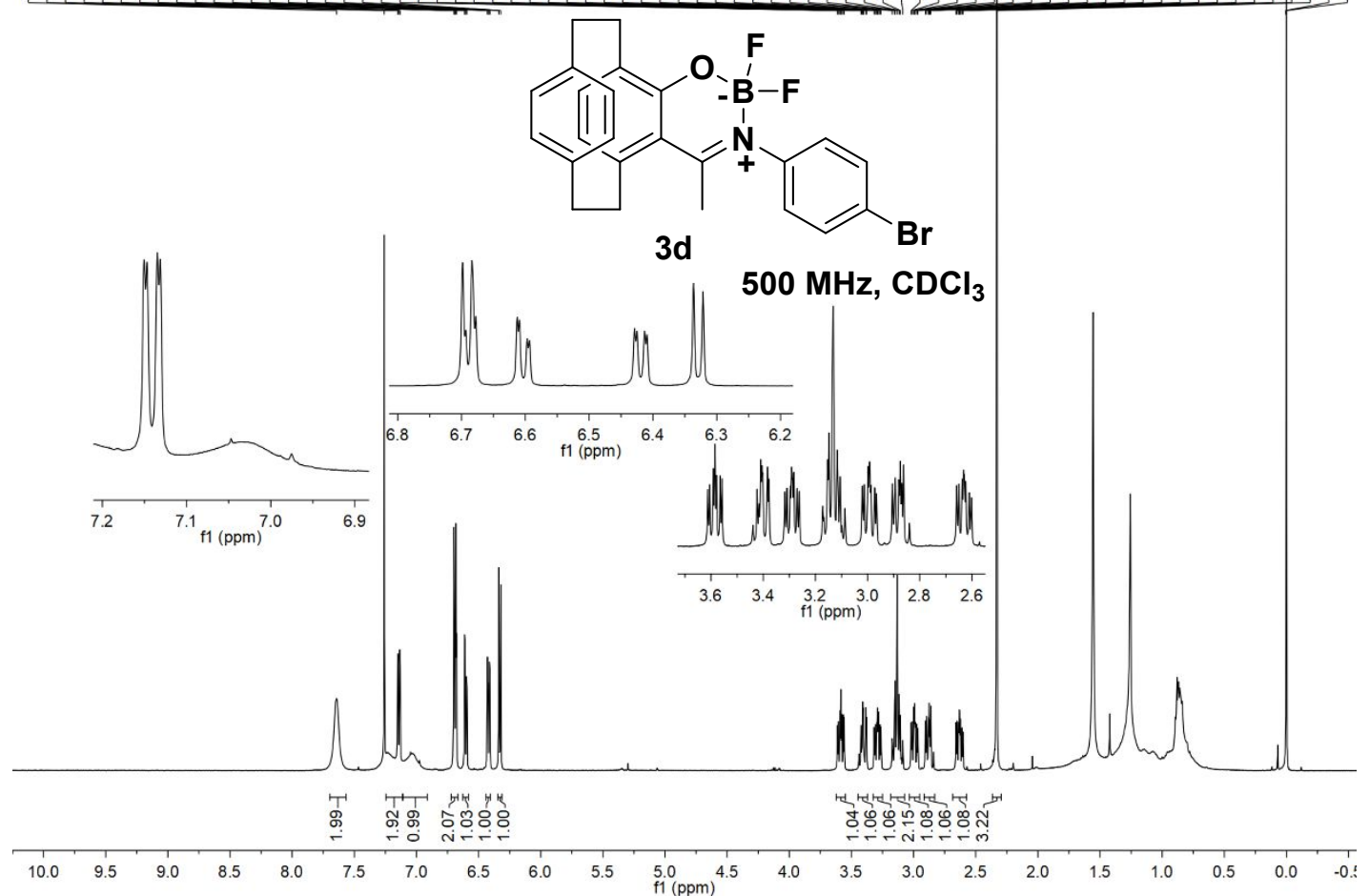

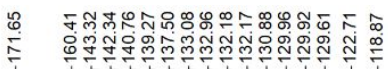
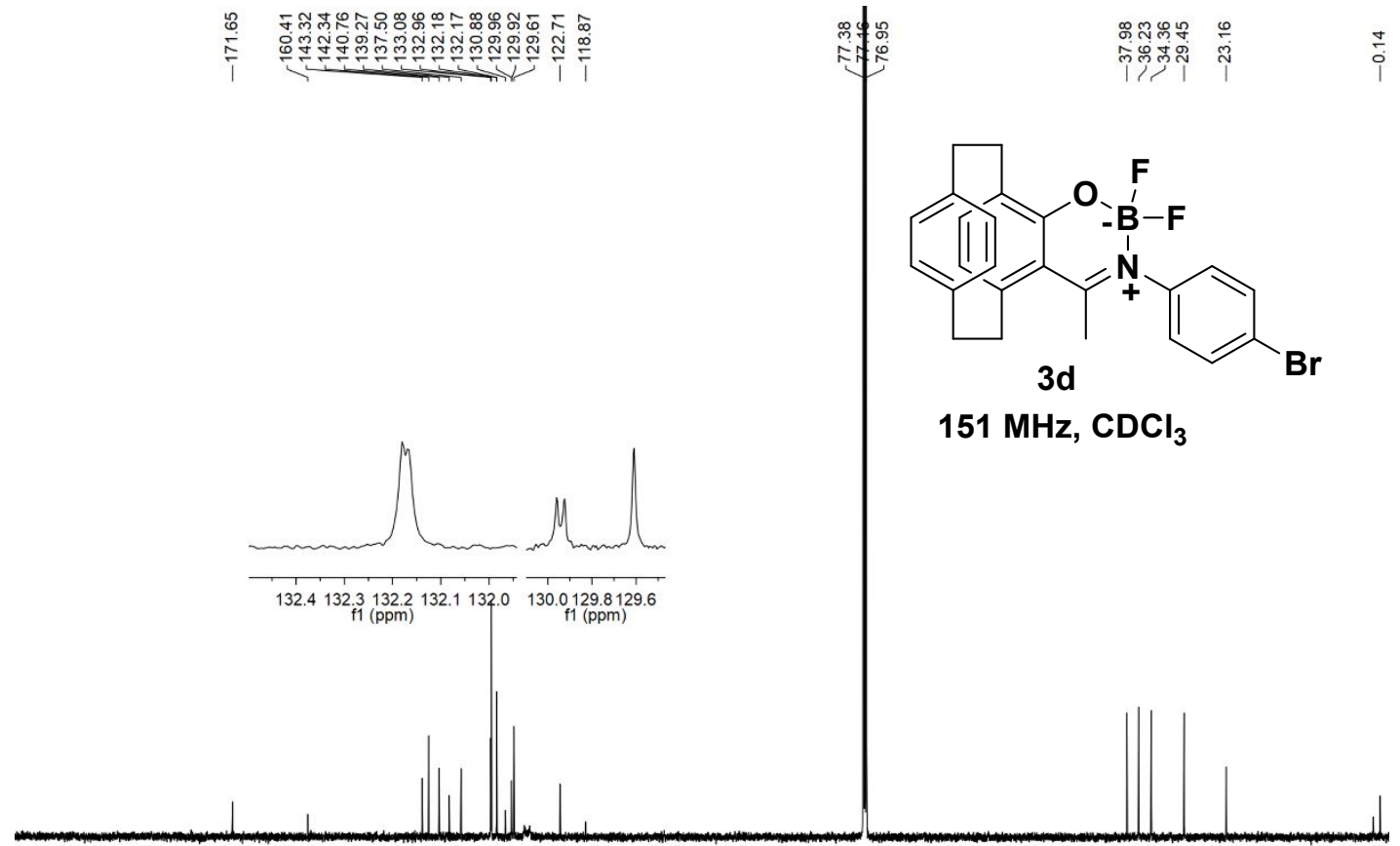

$151 \mathrm{MHz}, \mathrm{CDCl}_{3}$

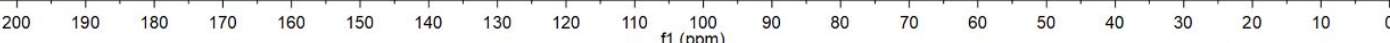




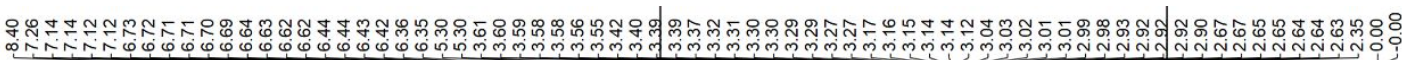

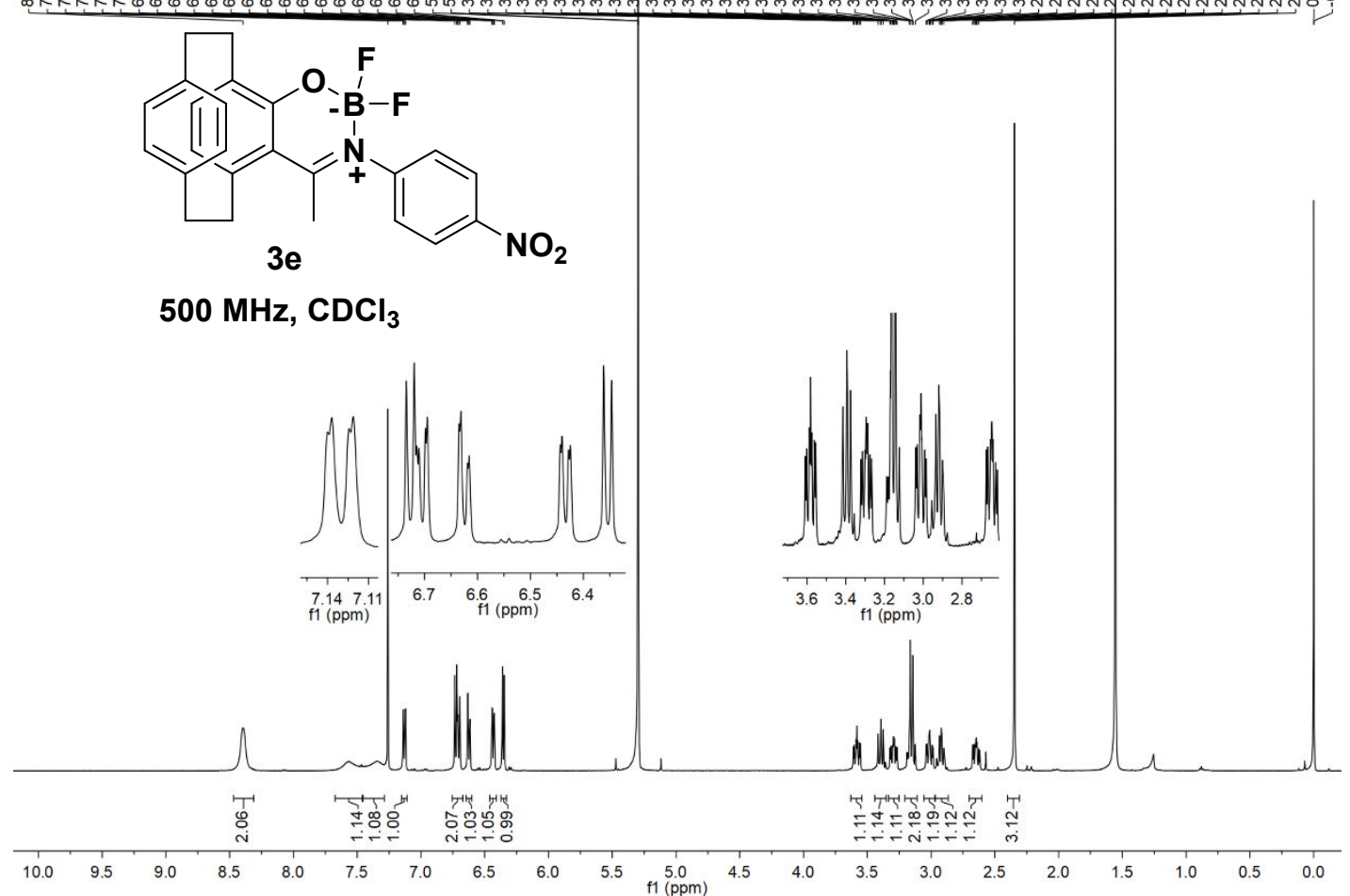

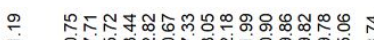

I
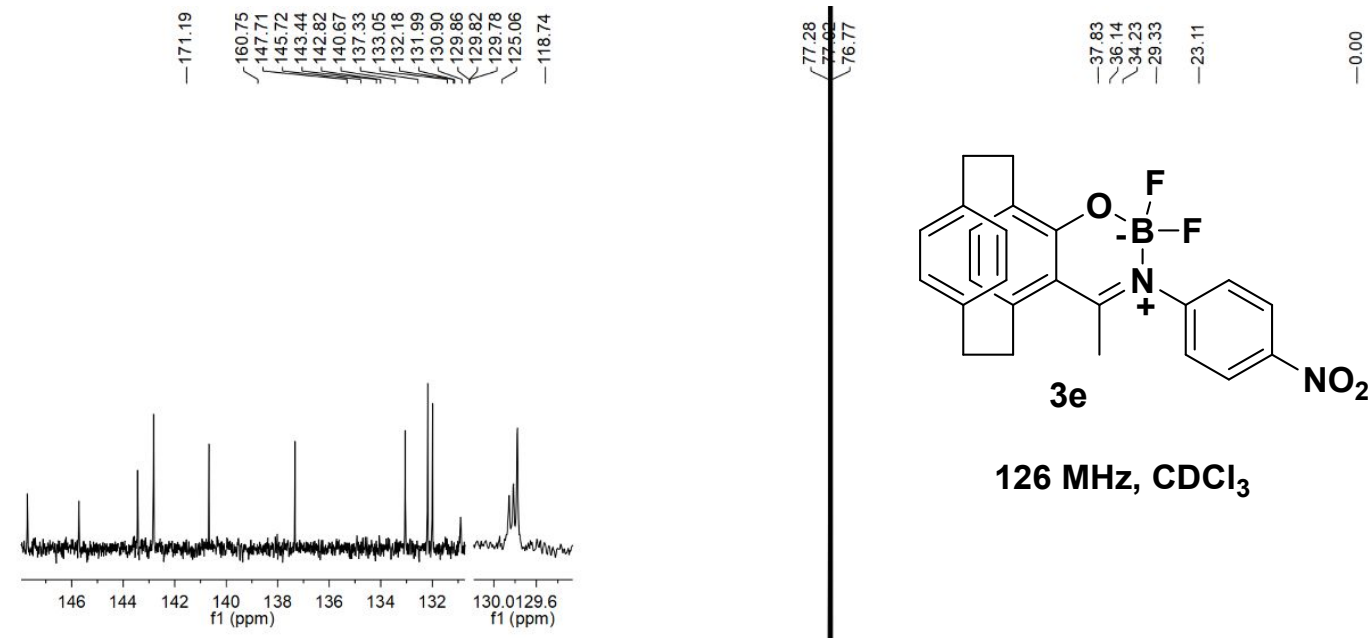

$126 \mathrm{MHz}, \mathrm{CDCl}_{3}$

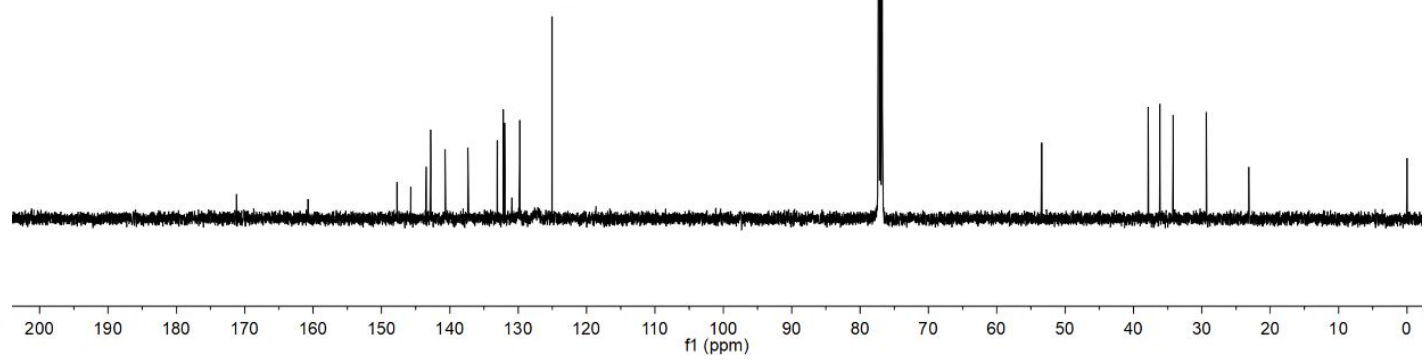




\subsection{HRMS Spectra}
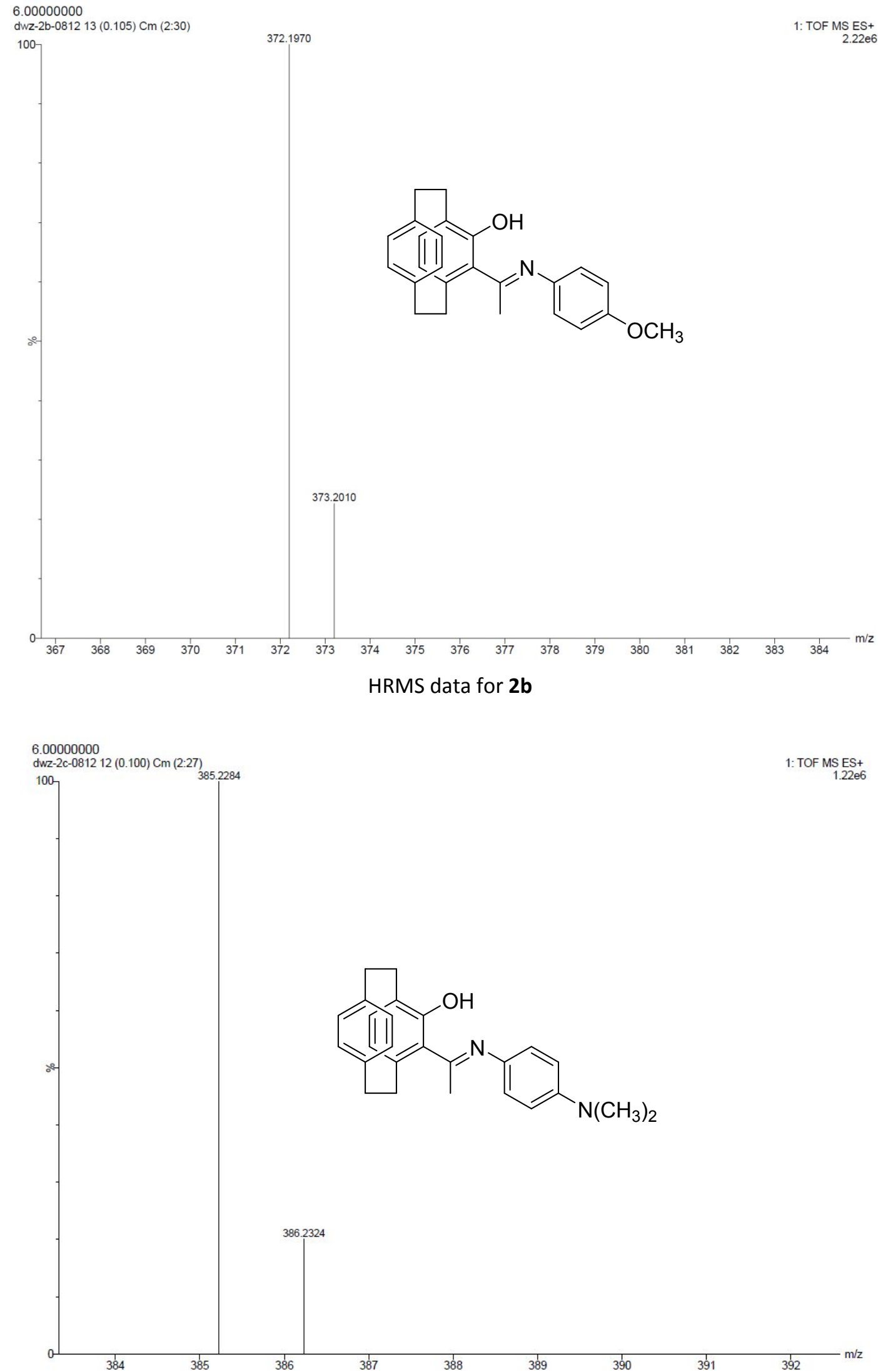

HRMS data for $\mathbf{2 c}$ 


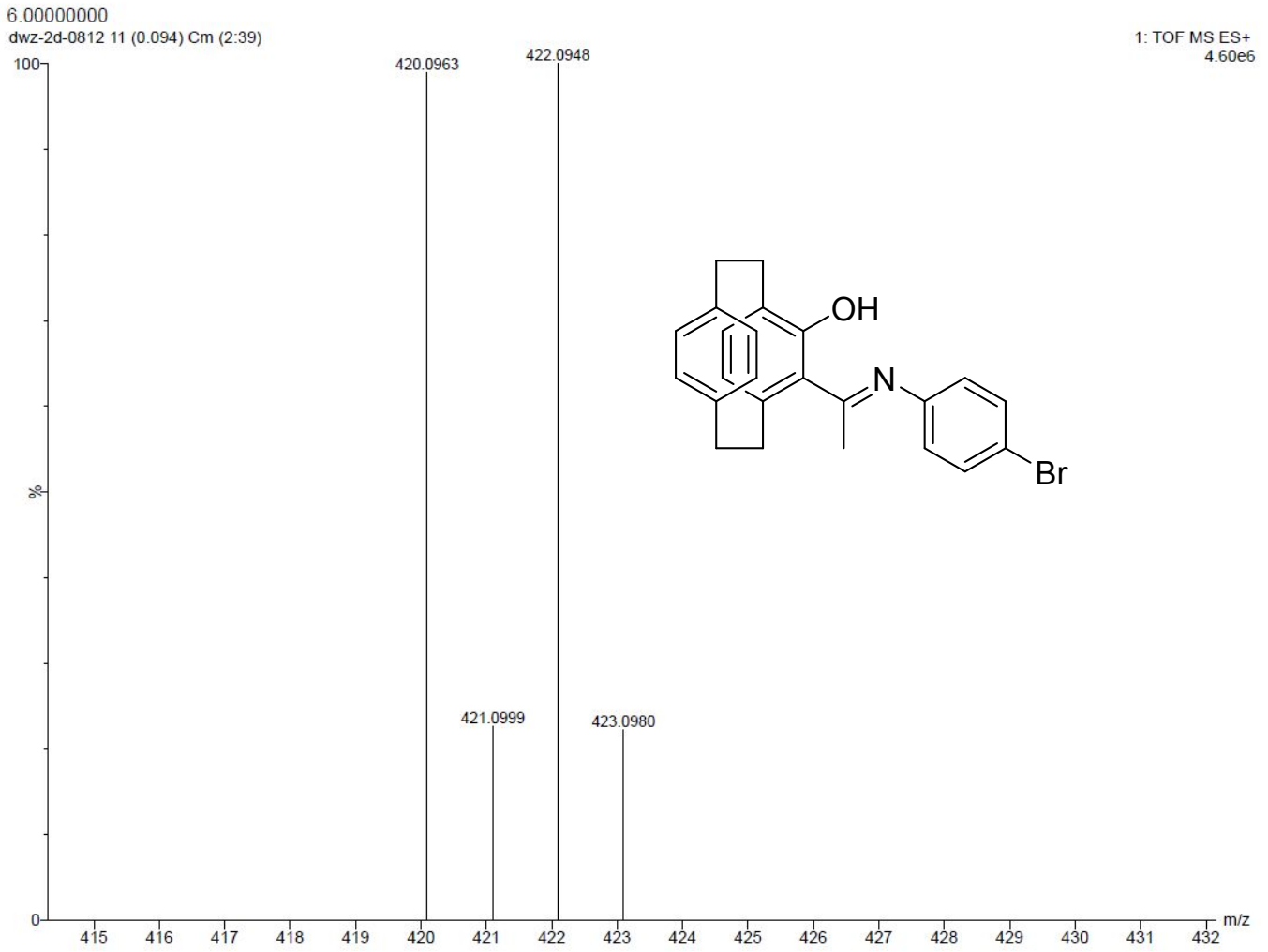

HRMS data for $\mathbf{2 d}$

6.00000000

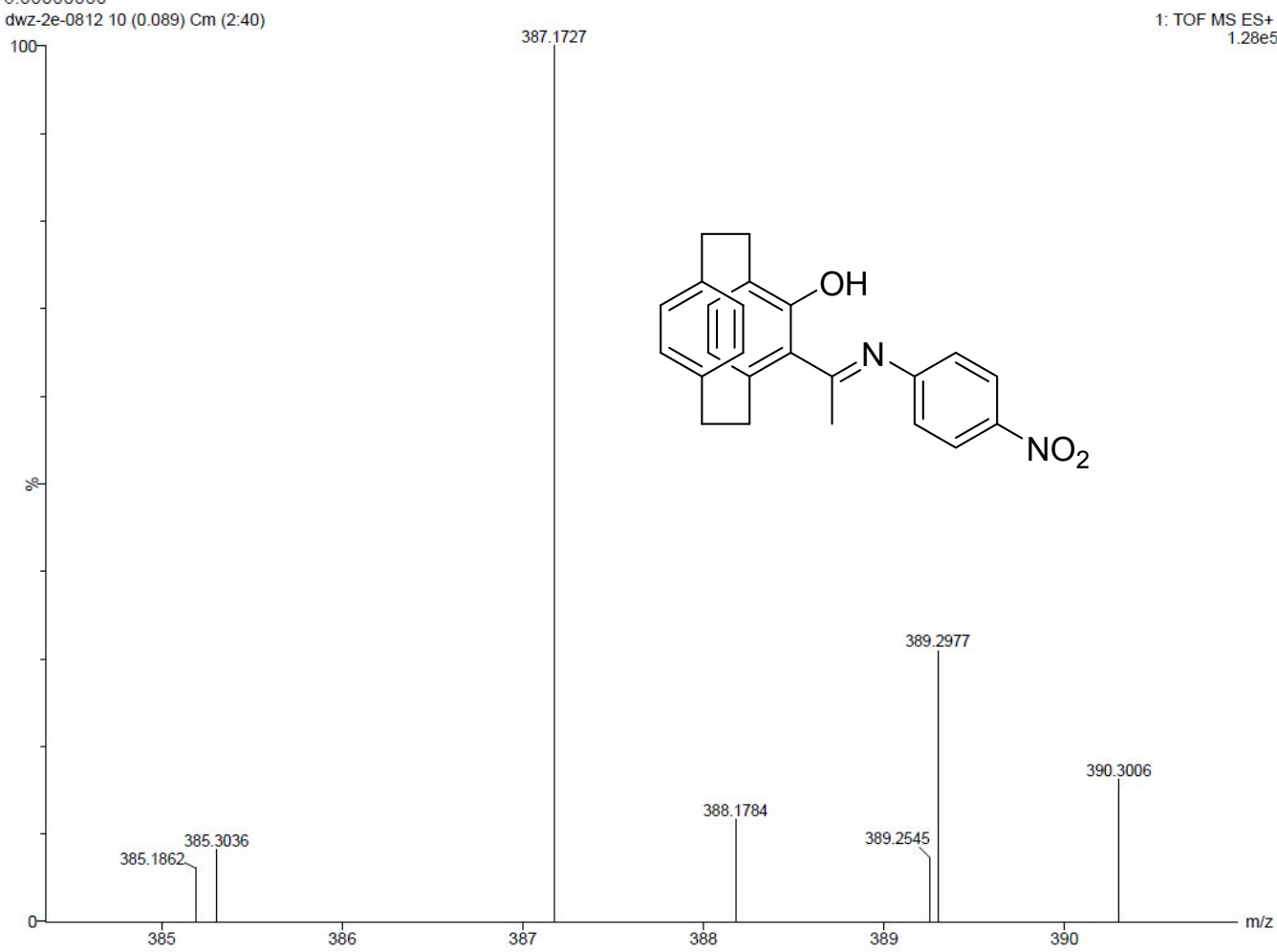

HRMS data for $\mathbf{2 e}$ 

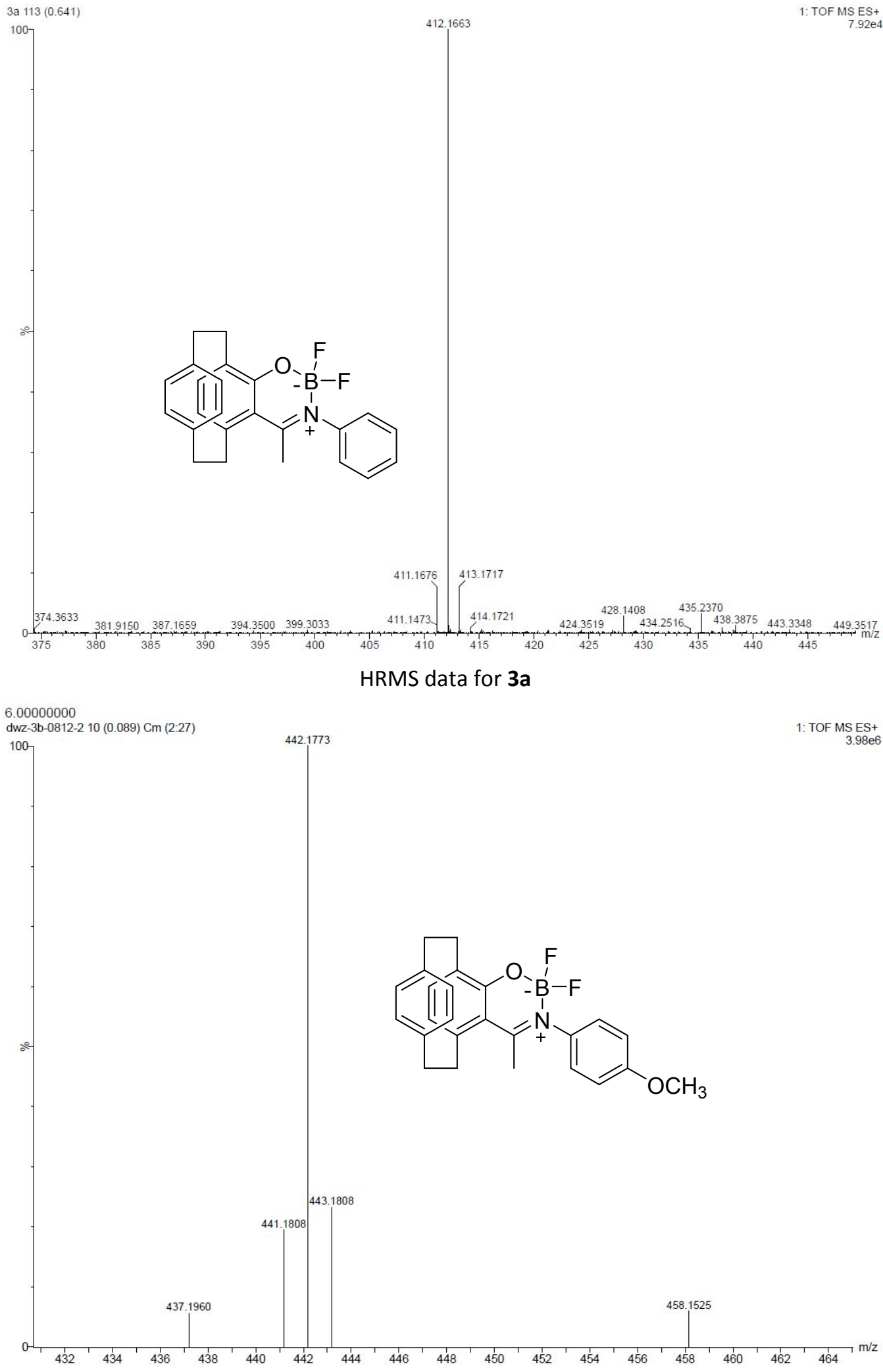

HRMS data for $\mathbf{3 b}$ 

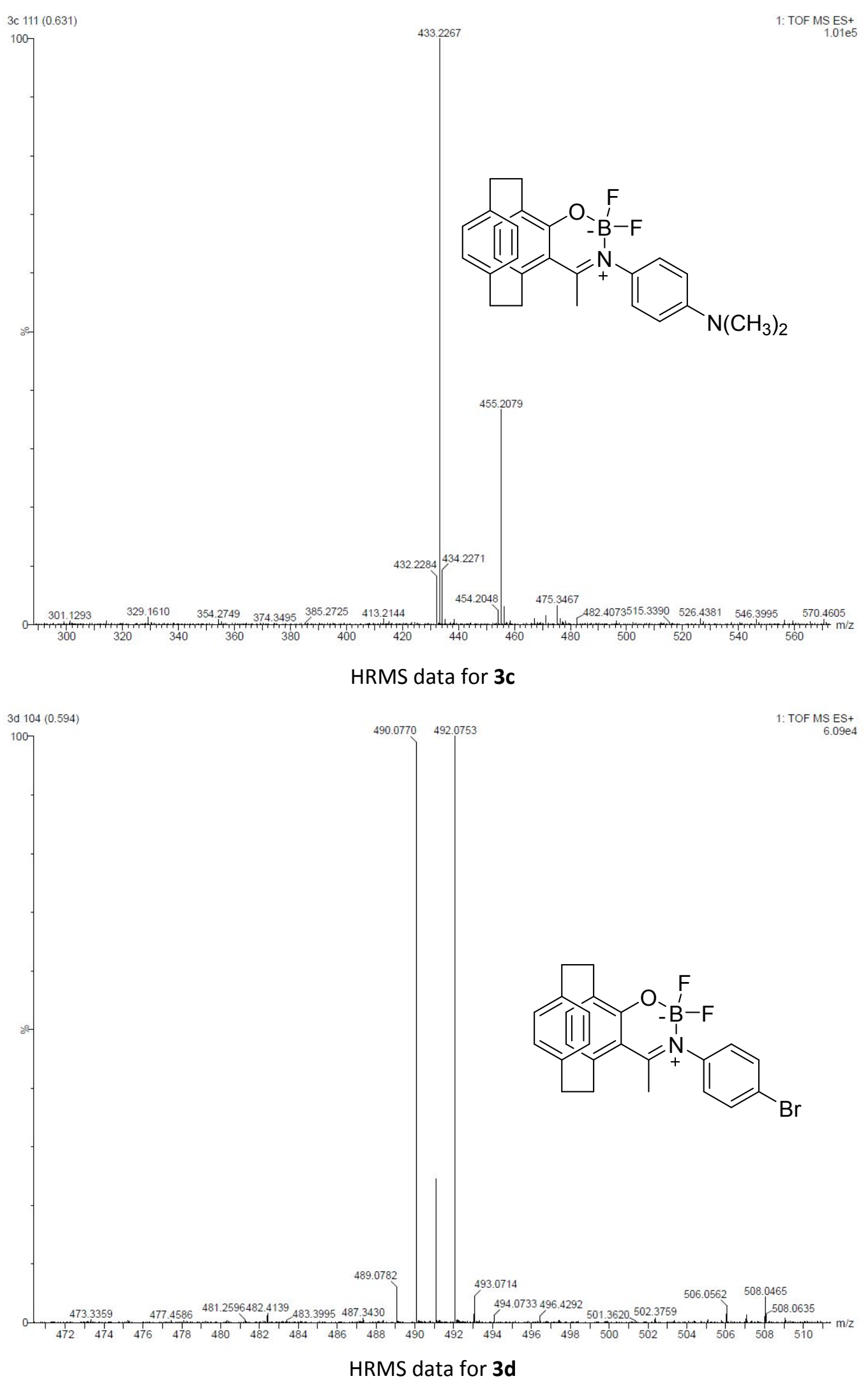


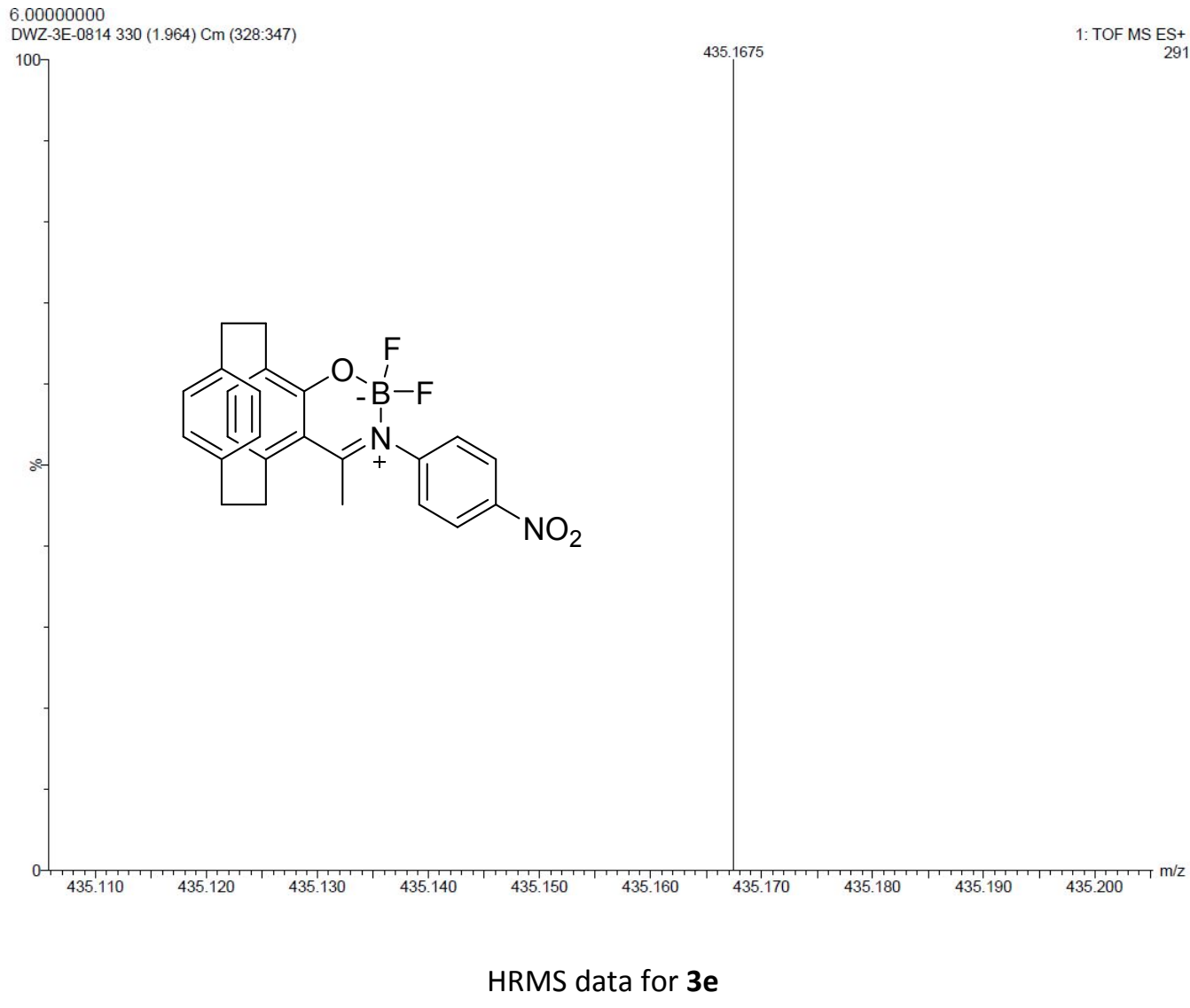

\section{Coordinates of optimized structures}

Coordinates of $\left(R_{p}\right) /\left(S_{p}\right)$-3a in the $\mathrm{S}_{0}$ state

$\begin{array}{llll}\mathrm{C} & 0.81279 & -0.81797 & -0.97655 \\ \mathrm{C} & 0.36039 & 0.53661 & -0.99996 \\ \mathrm{C} & 1.32124 & 1.57192 & -1.27305 \\ \mathrm{C} & 2.49941 & 1.18754 & -1.89289 \\ \mathrm{C} & 2.88963 & -0.16262 & -1.95344 \\ \mathrm{C} & 2.13673 & -1.16294 & -1.3599 \\ \mathrm{C} & 2.76466 & -2.46602 & -0.9076 \\ \mathrm{C} & 1.31807 & 2.9432 & -0.60971 \\ \mathrm{C} & 3.58703 & -2.34016 & 0.46804 \\ \mathrm{C} & 1.64279 & 2.88361 & 0.95573 \\ \mathrm{C} & 2.14409 & -0.75701 & 1.80982 \\ \mathrm{C} & 1.66039 & 0.54748 & 1.90698 \\ \mathrm{C} & 2.38014 & 1.6289 & 1.37869 \\ \mathrm{C} & 3.7153 & 1.39143 & 1.01919 \\ \mathrm{C} & 4.19515 & 0.08454 & 0.90687\end{array}$




\begin{tabular}{cccc} 
C & 3.35725 & -1.01309 & 1.15891 \\
$\mathrm{C}$ & -1.04387 & 0.78391 & -0.7612 \\
$\mathrm{H}$ & 3.23024 & 1.9461 & -2.15909 \\
$\mathrm{H}$ & 3.88652 & -0.39938 & -2.31879 \\
$\mathrm{H}$ & 3.45238 & -2.83247 & -1.67709 \\
$\mathrm{H}$ & 1.98782 & -3.22206 & -0.77987 \\
$\mathrm{H}$ & 2.096 & 3.54058 & -1.09513 \\
$\mathrm{H}$ & 0.38672 & 3.5013 & -0.72305 \\
$\mathrm{H}$ & 3.29281 & -3.17807 & 1.10962 \\
$\mathrm{H}$ & 4.65176 & -2.4645 & 0.24314 \\
$\mathrm{H}$ & 0.69614 & 2.93913 & 1.50418 \\
$\mathrm{H}$ & 2.20532 & 3.79346 & 1.19664 \\
$\mathrm{H}$ & 1.48681 & -1.57732 & 2.08248 \\
$\mathrm{H}$ & 0.64477 & 0.69826 & 2.26496 \\
$\mathrm{H}$ & 4.34079 & 2.21691 & 0.68478 \\
$\mathrm{H}$ & 5.18304 & -0.0819 & 0.48162 \\
$\mathrm{C}$ & -1.6863 & 2.08587 & -1.17183 \\
$\mathrm{H}$ & -1.79027 & 2.76063 & -0.31462 \\
$\mathrm{H}$ & -1.09891 & 2.58325 & -1.94189 \\
$\mathrm{H}$ & -2.69085 & 1.90396 & -1.55901 \\
$\mathrm{O}$ & 0.01493 & -1.80129 & -0.56936 \\
$\mathrm{C}$ & -3.21659 & 0.01152 & -0.04282 \\
$\mathrm{C}$ & -3.64559 & 0.62134 & 1.13852 \\
$\mathrm{C}$ & -4.14385 & -0.47677 & -0.96758 \\
$\mathrm{C}$ & -5.01259 & 0.7661 & 1.38282 \\
$\mathrm{H}$ & -2.91207 & 0.96899 & 1.85919 \\
$\mathrm{C}$ & -5.50817 & -0.32394 & -0.71793 \\
$\mathrm{H}$ & -3.7939 & -0.96864 & -1.86922 \\
$\mathrm{C}$ & -5.94489 & 0.2975 & 0.45502 \\
$\mathrm{H}$ & -5.3457 & 1.2414 & 2.30093 \\
$\mathrm{H}$ & -6.22961 & -0.69713 & -1.43909 \\
$\mathrm{H}$ & -7.00795 & 0.41073 & 0.64707 \\
$\mathrm{~B}$ & -1.20774 & -1.5646 & 0.19365 \\
$\mathrm{~F}$ & -0.95304 & -1.49226 & 1.56635 \\
$\mathrm{~F}$ & -2.11658 & -2.56766 & -0.08969 \\
$\mathrm{~N}$ & -1.80361 & -0.17662 & -0.26829 \\
$\mathrm{C}$ & & \\
\hline & -1.56 &
\end{tabular}

Coordinates of $\left(R_{p}\right) /\left(S_{p}\right)$-3a in the $\mathrm{S}_{1}$ state

$\begin{array}{lrrr}\mathrm{C} & 0.973825 & -0.910198 & -1.027845 \\ \mathrm{C} & 0.442993 & 0.405012 & -1.216189 \\ \mathrm{C} & 1.398133 & 1.434285 & -1.344095 \\ \mathrm{C} & 2.730880 & 1.090107 & -1.774514 \\ \mathrm{C} & 3.204669 & -0.202096 & -1.697931 \\ \mathrm{C} & 2.377880 & -1.191476 & -1.127062 \\ \mathrm{C} & 2.962554 & -2.432300 & -0.487183 \\ & & \\ & & & \\ & & & \end{array}$




\begin{tabular}{|c|c|c|c|}
\hline $\mathrm{C}$ & 1.200824 & 2.861574 & -0.846439 \\
\hline $\mathrm{C}$ & 3.615740 & -2.103559 & 0.923881 \\
\hline $\mathrm{C}$ & 1.150141 & 2.941067 & 0.732770 \\
\hline $\mathrm{C}$ & 1.796632 & -0.587699 & 1.864451 \\
\hline $\mathrm{C}$ & 1.195878 & 0.670626 & 1.840959 \\
\hline $\mathrm{C}$ & 1.898316 & 1.776444 & 1.352139 \\
\hline $\mathrm{C}$ & 3.298398 & 1.647600 & 1.212499 \\
\hline $\mathrm{C}$ & 3.909600 & 0.394075 & 1.277198 \\
\hline $\mathrm{C}$ & 3.134281 & -0.758659 & 1.439237 \\
\hline $\mathrm{C}$ & -0.986586 & 0.626036 & -1.082143 \\
\hline $\mathrm{H}$ & 3.390741 & 1.896141 & -2.084206 \\
\hline $\mathrm{H}$ & 4.240958 & -0.427013 & -1.931142 \\
\hline $\mathrm{H}$ & 3.729860 & -2.869293 & -1.136048 \\
\hline $\mathrm{H}$ & 2.175393 & -3.176005 & -0.356265 \\
\hline $\mathrm{H}$ & 2.050558 & 3.457356 & -1.194152 \\
\hline $\mathrm{H}$ & 0.302773 & 3.339563 & -1.243843 \\
\hline $\mathrm{H}$ & 3.370113 & -2.914021 & 1.618687 \\
\hline $\mathrm{H}$ & 4.705155 & -2.084089 & 0.821337 \\
\hline $\mathrm{H}$ & 0.108108 & 2.917063 & 1.065864 \\
\hline $\mathrm{H}$ & 1.564510 & 3.909864 & 1.034927 \\
\hline $\mathrm{H}$ & 1.179277 & -1.454159 & 2.078017 \\
\hline $\mathrm{H}$ & 0.130345 & 0.743520 & 2.037361 \\
\hline $\mathrm{H}$ & 3.892821 & 2.517713 & 0.944192 \\
\hline $\mathrm{H}$ & 4.966569 & 0.305510 & 1.039935 \\
\hline $\mathrm{C}$ & -1.645970 & 1.798247 & -1.758670 \\
\hline $\mathrm{H}$ & -1.820214 & 2.671542 & -1.114050 \\
\hline $\mathrm{H}$ & -1.039740 & 2.132667 & -2.606026 \\
\hline $\mathrm{H}$ & -2.627609 & 1.499692 & -2.142379 \\
\hline $\mathrm{O}$ & 0.182846 & -1.905313 & -0.668394 \\
\hline $\mathrm{C}$ & -3.083871 & -0.049249 & -0.079507 \\
\hline $\mathrm{C}$ & -3.335833 & 1.096776 & 0.698299 \\
\hline $\mathrm{C}$ & -4.156600 & -0.908328 & -0.381039 \\
\hline $\mathrm{C}$ & -4.624757 & 1.386536 & 1.140492 \\
\hline $\mathrm{H}$ & -2.508701 & 1.744426 & 0.972029 \\
\hline $\mathrm{C}$ & -5.442358 & -0.612860 & 0.069823 \\
\hline $\mathrm{H}$ & -3.971056 & -1.796722 & -0.970777 \\
\hline $\mathrm{C}$ & -5.688476 & 0.535647 & 0.826988 \\
\hline $\mathrm{H}$ & -4.795340 & 2.274355 & 1.744066 \\
\hline $\mathrm{H}$ & -6.259567 & -1.284595 & -0.180138 \\
\hline $\mathrm{H}$ & -6.693264 & 0.762216 & 1.172194 \\
\hline B & -1.177472 & -1.728229 & -0.097410 \\
\hline $\mathrm{F}$ & -1.080019 & -1.797671 & 1.302016 \\
\hline $\mathrm{F}$ & -1.949361 & -2.785128 & -0.573518 \\
\hline $\mathrm{N}$ & -1.765776 & -0.354648 & -0.518368 \\
\hline
\end{tabular}


Coordinates of $\left(R_{p}\right) /\left(S_{p}\right)-\mathbf{3} \mathbf{c}$ in the $\mathrm{S}_{0}$ state

C

C

C

C

C

C

C

C

C

C

C

C

C

C

C

C

C

$\mathrm{H}$

$\mathrm{H}$

$\mathrm{H}$

$\mathrm{H}$

$\mathrm{H}$

$\mathrm{H}$

$\mathrm{H}$

$\mathrm{H}$

$\mathrm{H}$

$\mathrm{H}$

$\mathrm{H}$

$\mathrm{H}$

$\mathrm{H}$

$\mathrm{H}$

C

$\mathrm{H}$

$\mathrm{H}$

$\mathrm{H}$

O

C

C

C

C

$\mathrm{H}$

C

$\mathrm{H}$ $\begin{array}{lll}1.74345 & -0.91593 & -0.9013\end{array}$

$\begin{array}{lll}1.24224 & 0.40712 & -1.08526\end{array}$

$\begin{array}{lll}2.17696 & 1.45161 & -1.40489\end{array}$

$\begin{array}{lll}3.40554 & 1.06228 & -1.9154\end{array}$

$\begin{array}{lll}3.85038 & -0.26842 & -1.81892\end{array}$

$\begin{array}{lll}3.10066 & -1.23759 & -1.1716\end{array}$

$\begin{array}{lll}3.74845 & -2.46083 & -0.55403\end{array}$

$\begin{array}{lll}2.07675 & 2.87935 & -0.88308\end{array}$

$\begin{array}{lll}4.4853 & -2.16087 & 0.84178\end{array}$

$\begin{array}{rrr}2.30384 & 2.9889 & 0.69655\end{array}$

$\begin{array}{lll}2.89646 & -0.52618 & 1.93376\end{array}$

$\begin{array}{lll}2.35424 & 0.75773 & 1.87347\end{array}$

$\begin{array}{lll}3.062 & 1.81661 & 1.28669\end{array}$

$\begin{array}{lll}4.42628 & 1.6088 & 1.03333\end{array}$

$\begin{array}{lll}4.96494 & 0.32101 & 1.07737\end{array}$

$\begin{array}{lll}4.15827 & -0.78579 & 1.38488\end{array}$

$\begin{array}{lll}-0.1841 & 0.61524 & -0.95249\end{array}$

$\begin{array}{llll}4.12119 & 1.82337 & -2.21405\end{array}$

$\begin{array}{lll}4.87586 & -0.49594 & -2.10198\end{array}$

$\begin{array}{lll}4.4917 & -2.87588 & -1.24313\end{array}$

$\begin{array}{llll}2.9924 & -3.23073 & -0.38962\end{array}$

$\begin{array}{llll}2.85819 & 3.46326 & -1.37926\end{array}$

$\begin{array}{lll}1.13165 & 3.37741 & -1.10744\end{array}$

$\begin{array}{lll}4.1939 & -2.94631 & 1.548\end{array}$

$\begin{array}{lll}5.5657 & -2.2526 & 0.6864\end{array}$

$\begin{array}{lll}1.32304 & 3.05643 & 1.18025\end{array}$

$\begin{array}{lll}2.81465 & 3.94228 & 0.877\end{array}$

$\begin{array}{lll}2.25513 & -1.34654 & 2.24188\end{array}$

$\begin{array}{lll}1.31138 & 0.89283 & 2.15001\end{array}$

$\begin{array}{lll}5.0376 & 2.42723 & 0.6578\end{array}$

$\begin{array}{lll}5.98396 & 0.16141 & 0.72993\end{array}$

$\begin{array}{lll}-0.8432 & 1.83114 & -1.55678\end{array}$

$\begin{array}{lll}-1.02321 & 2.6081 & -0.80602\end{array}$

$\begin{array}{lll}-0.22449 & 2.24859 & -2.35039\end{array}$

$\begin{array}{lll}-1.81604 & 1.55673 & -1.97076\end{array}$

$\begin{array}{lll}0.95915 & -1.8883 & -0.4439\end{array}$

$\begin{array}{lll}-2.36324 & -0.14634 & -0.25632\end{array}$

$\begin{array}{lll}-2.87447 & 0.73185 & 0.70295\end{array}$

$\begin{array}{lll}-3.25514 & -0.90737 & -1.01714\end{array}$

$\begin{array}{lll}-4.24606 & 0.87487 & 0.88142\end{array}$

$\begin{array}{lll}-2.19228 & 1.29935 & 1.32964\end{array}$

$\begin{array}{lll}-4.62791 & -0.76549 & -0.8531\end{array}$

$\begin{array}{lll}-2.87147 & -1.61593 & -1.74319\end{array}$ 


$\begin{array}{lccc}\text { C } & -5.16683 & 0.14008 & 0.09467 \\ \mathrm{H} & -4.59751 & 1.55763 & 1.64496 \\ \mathrm{H} & -5.28345 & -1.37248 & -1.46504 \\ \mathrm{~B} & -0.31679 & -1.62931 & 0.22083 \\ \mathrm{~F} & -0.14538 & -1.42007 & 1.59314 \\ \mathrm{~F} & -1.16134 & -2.70133 & -0.01227 \\ \mathrm{~N} & -0.93834 & -0.31894 & -0.40287 \\ \mathrm{C} & -7.44038 & -0.60749 & -0.44359 \\ \mathrm{H} & -8.46811 & -0.31654 & -0.22201 \\ \mathrm{H} & -7.30726 & -0.55398 & -1.53058 \\ \mathrm{H} & -7.30326 & -1.6548 & -0.13398 \\ \mathrm{C} & -7.04737 & 1.09284 & 1.34876 \\ \mathrm{H} & -6.77349 & 0.67628 & 2.33026 \\ \mathrm{H} & -6.67775 & 2.12392 & 1.30186 \\ \mathrm{H} & -8.13577 & 1.12903 & 1.28411 \\ \mathrm{~N} & -6.53213 & 0.30185 & 0.2406\end{array}$

Coordinates of $\left(R_{p}\right) /\left(S_{p}\right)-3 \mathbf{c}$ in the $\mathrm{S}_{1}$ state

\begin{tabular}{|c|c|c|c|}
\hline $\mathrm{C}$ & 1.783095 & -0.870149 & -0.945030 \\
\hline $\mathrm{C}$ & 1.244042 & 0.465114 & -1.128847 \\
\hline $\mathrm{C}$ & 2.213622 & 1.512000 & -1.404822 \\
\hline $\mathrm{C}$ & 3.455871 & 1.124820 & -1.904070 \\
\hline $\mathrm{C}$ & 3.897282 & -0.200324 & -1.863072 \\
\hline $\mathrm{C}$ & 3.114951 & -1.178916 & -1.221255 \\
\hline $\mathrm{C}$ & 3.765558 & -2.417129 & -0.634199 \\
\hline $\mathrm{C}$ & 2.129532 & 2.913044 & -0.814064 \\
\hline $\mathrm{C}$ & 4.441050 & -2.169716 & 0.800176 \\
\hline $\mathrm{C}$ & 2.224190 & 2.965093 & 0.779207 \\
\hline $\mathrm{C}$ & 2.747223 & -0.614224 & 1.849220 \\
\hline $\mathrm{C}$ & 2.195443 & 0.667032 & 1.827863 \\
\hline $\mathrm{C}$ & 2.935035 & 1.764285 & 1.365947 \\
\hline $\mathrm{C}$ & 4.316565 & 1.581019 & 1.208189 \\
\hline $\mathrm{C}$ & 4.865107 & 0.297687 & 1.212732 \\
\hline $\mathrm{C}$ & 4.052016 & -0.831624 & 1.390851 \\
\hline $\mathrm{C}$ & -0.131973 & 0.684595 & -0.966011 \\
\hline $\mathrm{H}$ & 4.167754 & 1.901501 & -2.180845 \\
\hline $\mathrm{H}$ & 4.914263 & -0.441852 & -2.160361 \\
\hline $\mathrm{H}$ & 4.544226 & -2.798822 & -1.306190 \\
\hline $\mathrm{H}$ & 3.018464 & -3.206130 & -0.524940 \\
\hline $\mathrm{H}$ & 2.971933 & 3.490225 & -1.214026 \\
\hline $\mathrm{H}$ & 1.227371 & 3.469726 & -1.084543 \\
\hline $\mathrm{H}$ & 4.142416 & -2.994662 & 1.458367 \\
\hline $\mathrm{H}$ & 5.530545 & -2.227115 & 0.691809 \\
\hline $\mathrm{H}$ & 1.208637 & 3.016518 & 1.189350 \\
\hline $\mathrm{H}$ & 2.720560 & 3.908382 & 1.042877 \\
\hline
\end{tabular}




\begin{tabular}{|c|c|c|c|}
\hline $\mathrm{H}$ & 2.090951 & -1.456592 & 2.045042 \\
\hline $\mathrm{H}$ & 1.129843 & 0.773660 & 2.015114 \\
\hline $\mathrm{H}$ & 4.944325 & 2.424957 & 0.926726 \\
\hline $\mathrm{H}$ & 5.908952 & 0.167109 & 0.932332 \\
\hline $\mathrm{C}$ & -0.845892 & 1.909665 & -1.460428 \\
\hline $\mathrm{H}$ & -1.040044 & 2.659406 & -0.675745 \\
\hline $\mathrm{H}$ & -0.261083 & 2.395446 & -2.244142 \\
\hline $\mathrm{H}$ & -1.819035 & 1.657095 & -1.898123 \\
\hline $\mathrm{O}$ & 0.987319 & -1.861762 & -0.465400 \\
\hline $\mathrm{C}$ & -2.306284 & -0.172964 & -0.270888 \\
\hline $\mathrm{C}$ & -2.826988 & 0.474277 & 0.877726 \\
\hline $\mathrm{C}$ & -3.228005 & -0.707833 & -1.206262 \\
\hline $\mathrm{C}$ & -4.181475 & 0.634208 & 1.065090 \\
\hline $\mathrm{H}$ & -2.126143 & 0.863913 & 1.606746 \\
\hline $\mathrm{C}$ & -4.587159 & -0.595948 & -1.023605 \\
\hline $\mathrm{H}$ & -2.831834 & -1.227396 & -2.069437 \\
\hline $\mathrm{C}$ & -5.109268 & 0.094628 & 0.116520 \\
\hline $\mathrm{H}$ & -4.538769 & 1.157909 & 1.940462 \\
\hline $\mathrm{H}$ & -5.258195 & -1.038955 & -1.746024 \\
\hline B & -0.289103 & -1.616914 & 0.169266 \\
\hline $\mathrm{F}$ & -0.163536 & -1.457565 & 1.573225 \\
\hline $\mathrm{F}$ & -1.131048 & -2.717855 & -0.063276 \\
\hline $\mathrm{N}$ & -0.930895 & -0.341923 & -0.448213 \\
\hline $\mathrm{C}$ & -7.405394 & -0.352456 & -0.655783 \\
\hline $\mathrm{H}$ & -8.405248 & -0.018567 & -0.392618 \\
\hline $\mathrm{H}$ & -7.181292 & -0.022144 & -1.671211 \\
\hline $\mathrm{H}$ & -7.365666 & -1.444815 & -0.608957 \\
\hline $\mathrm{C}$ & -6.996293 & 0.886966 & 1.491276 \\
\hline $\mathrm{H}$ & -6.810800 & 0.273040 & 2.377538 \\
\hline $\mathrm{H}$ & -6.543900 & 1.870471 & 1.627101 \\
\hline $\mathrm{H}$ & -8.067779 & 1.010415 & 1.360237 \\
\hline $\mathrm{N}$ & -6.446723 & 0.229897 & 0.295642 \\
\hline
\end{tabular}

Coordinates of $\left(R_{p}\right) /\left(S_{p}\right)-\mathbf{3 d}$ in the $\mathrm{S}_{0}$ state

$\begin{array}{llrl}\mathrm{C} & 2.02005 & -0.84794 & -0.95551 \\ \mathrm{C} & 1.51901 & 0.48685 & -1.04586 \\ \mathrm{C} & 2.4542 & 1.55108 & -1.29967 \\ \mathrm{C} & 3.67795 & 1.19447 & -1.84215 \\ \mathrm{C} & 4.12212 & -0.14074 & -1.83637 \\ \mathrm{C} & 3.37572 & -1.15135 & -1.25259 \\ \mathrm{C} & 4.0243 & -2.41387 & -0.72166 \\ \mathrm{C} & 2.36219 & 2.94167 & -0.6849 \\ \mathrm{C} & 4.76457 & -2.21042 & 0.68997 \\ \mathrm{C} & 2.60108 & 2.94638 & 0.89688 \\ \mathrm{C} & 3.18526 & -0.64553 & 1.89344\end{array}$




\begin{tabular}{|c|c|c|c|}
\hline $\mathrm{C}$ & 2.64829 & 0.64145 & 1.92109 \\
\hline $\mathrm{C}$ & 3.35758 & 1.73419 & 1.40227 \\
\hline $\mathrm{C}$ & 4.71979 & 1.5384 & 1.1289 \\
\hline $\mathrm{C}$ & 5.25407 & 0.24864 & 1.08659 \\
\hline $\mathrm{C}$ & 4.44436 & -0.87317 & 1.32388 \\
\hline $\mathrm{C}$ & 0.09673 & 0.6857 & -0.89402 \\
\hline $\mathrm{H}$ & 4.39358 & 1.97229 & -2.09342 \\
\hline $\mathrm{H}$ & 5.14619 & -0.34869 & -2.13857 \\
\hline $\mathrm{H}$ & 4.76556 & -2.78078 & -1.43944 \\
\hline $\mathrm{H}$ & 3.26834 & -3.19299 & -0.60857 \\
\hline $\mathrm{H}$ & 3.14346 & 3.55254 & -1.14744 \\
\hline $\mathrm{H}$ & 1.41867 & 3.4585 & -0.86999 \\
\hline $\mathrm{H}$ & 4.47053 & -3.03979 & 1.34266 \\
\hline $\mathrm{H}$ & 5.84416 & -2.29599 & 0.52657 \\
\hline $\mathrm{H}$ & 1.62443 & 2.9877 & 1.39197 \\
\hline $\mathrm{H}$ & 3.11873 & 3.88315 & 1.13488 \\
\hline $\mathrm{H}$ & 2.54292 & -1.48264 & 2.1501 \\
\hline $\mathrm{H}$ & 1.6081 & 0.7636 & 2.21364 \\
\hline $\mathrm{H}$ & 5.33281 & 2.37769 & 0.80592 \\
\hline $\mathrm{H}$ & 6.2715 & 0.10881 & 0.72629 \\
\hline $\mathrm{C}$ & -0.56808 & 1.94851 & -1.38347 \\
\hline $\mathrm{H}$ & -0.72791 & 2.65439 & -0.56078 \\
\hline $\mathrm{H}$ & 0.03793 & 2.43319 & -2.14743 \\
\hline $\mathrm{H}$ & -1.54869 & 1.72026 & -1.80608 \\
\hline $\mathrm{O}$ & 1.23779 & -1.85023 & -0.56563 \\
\hline $\mathrm{C}$ & -2.0801 & -0.14876 & -0.26813 \\
\hline $\mathrm{C}$ & -2.59778 & 0.54948 & 0.82548 \\
\hline $\mathrm{C}$ & -2.94043 & -0.77081 & -1.17721 \\
\hline $\mathrm{C}$ & -3.97813 & 0.65404 & 0.99922 \\
\hline $\mathrm{H}$ & -1.92387 & 1.00342 & 1.5448 \\
\hline $\mathrm{C}$ & -4.32107 & -0.66676 & -1.01258 \\
\hline $\mathrm{H}$ & -2.53086 & -1.33226 & -2.00958 \\
\hline $\mathrm{C}$ & -4.82448 & 0.0471 & 0.07403 \\
\hline $\mathrm{H}$ & -4.38405 & 1.1962 & 1.84579 \\
\hline $\mathrm{H}$ & -4.99359 & -1.13992 & -1.7192 \\
\hline B & -0.03324 & -1.64343 & 0.12111 \\
\hline $\mathrm{F}$ & 0.13868 & -1.52638 & 1.50254 \\
\hline $\mathrm{F}$ & -0.88745 & -2.68696 & -0.18577 \\
\hline $\mathrm{N}$ & -0.65413 & -0.29002 & -0.41314 \\
\hline $\mathrm{Br}$ & -6.71998 & 0.19083 & 0.30322 \\
\hline
\end{tabular}

Coordinates of $\left(R_{p}\right) /\left(S_{p}\right)$-3d in the $\mathrm{S}_{1}$ state

$\begin{array}{lrrr}\mathrm{C} & 2.200425 & -0.930834 & -0.959865 \\ \mathrm{C} & 1.609915 & 0.339558 & -1.247903 \\ \mathrm{C} & 2.512753 & 1.422874 & -1.356416 \\ & & \end{array}$




$\begin{array}{lccc}\mathrm{C} & 3.889425 & 1.143562 & -1.663753 \\ \mathrm{C} & 4.430697 & -0.114871 & -1.492830 \\ \mathrm{C} & 3.620973 & -1.126973 & -0.939926 \\ \mathrm{C} & 4.225325 & -2.303197 & -0.200441 \\ \mathrm{C} & 2.195141 & 2.855553 & -0.938198 \\ \mathrm{C} & 4.763934 & -1.870406 & 1.227912 \\ \mathrm{C} & 2.010809 & 2.990403 & 0.624573 \\ \mathrm{C} & 2.787231 & -0.435611 & 1.962847 \\ \mathrm{C} & 2.113718 & 0.778513 & 1.840156 \\ \mathrm{C} & 2.782109 & 1.903243 & 1.347698 \\ \mathrm{C} & 4.198086 & 1.858978 & 1.317982 \\ \mathrm{C} & 4.879191 & 0.653801 & 1.489095 \\ \mathrm{C} & 4.164221 & -0.538515 & 1.642749 \\ \mathrm{C} & 0.166640 & 0.475664 & -1.252293 \\ \mathrm{H} & 4.530562 & 1.974533 & -1.946358 \\ \mathrm{H} & 5.494693 & -0.281733 & -1.629543 \\ \mathrm{H} & 5.055177 & -2.729891 & -0.774871 \\ \mathrm{H} & 3.469456 & -3.081173 & -0.083354 \\ \mathrm{H} & 3.038911 & 3.484361 & -1.239737 \\ \mathrm{H} & 1.310316 & 3.269205 & -1.427437 \\ \mathrm{H} & 4.527767 & -2.661378 & 1.948143 \\ \mathrm{H} & 5.853840 & -1.779244 & 1.193056 \\ \mathrm{H} & 0.950179 & 2.904541 & 0.879720 \\ \mathrm{H} & 2.333080 & 3.996121 & 0.919181 \\ \mathrm{H} & 2.211019 & -1.330573 & 2.172624 \\ \mathrm{H} & 1.034502 & 0.790412 & 1.955516 \\ \mathrm{H} & 4.755697 & 2.754798 & 1.056371 \\ \mathrm{H} & 5.954636 & 0.623286 & 1.338092 \\ \mathrm{C} & -0.494201 & 1.584130 & -2.030586 \\ \mathrm{H} & -0.771407 & 2.469322 & -1.440726 \\ \mathrm{H} & 0.166192 & 1.921636 & -2.834895 \\ \mathrm{H} & -1.419327 & 1.215508 & -2.486404 \\ \mathrm{O} & 1.441732 & -1.960548 & -0.618386 \\ \mathrm{C} & -1.977467 & -0.332087 & -0.465890 \\ \mathrm{C} & -2.412058 & 0.816023 & 0.223816 \\ \mathrm{C} & -2.936444 & -1.293589 & -0.836130 \\ \mathrm{C} & -3.759663 & 1.014730 & 0.512138 \\ \mathrm{H} & -1.683836 & 1.546664 & 0.559486 \\ \mathrm{C} & -4.285461 & -1.103674 & -0.546215 \\ \mathrm{H} & -2.617401 & -2.186570 & -1.357153 \\ \mathrm{C} & -4.688844 & 0.052397 & 0.120192 \\ \mathrm{H} & -5.079055 & 1.901477 & 1.049269 \\ \mathrm{H} & 0.039778 & -1.847334 & -0.845954 \\ & & & -0.157809 \\ & & \\ & & & \\ & & & \\ & & & \end{array}$




$\begin{array}{lccc}\mathrm{F} & 0.031515 & -1.760500 & 1.246531 \\ \mathrm{~F} & -0.629131 & -2.977775 & -0.578944 \\ \mathrm{~N} & -0.603979 & -0.544990 & -0.748418 \\ \mathrm{Br} & -6.548687 & 0.323300 & 0.505414\end{array}$

Coordinates of $\left(R_{p}\right) /\left(S_{p}\right)-3 \mathbf{e}$ in the $\mathrm{S}_{0}$ state

\begin{tabular}{|c|c|c|c|}
\hline $\mathrm{C}$ & 1.62435 & -0.86778 & -0.92333 \\
\hline $\mathrm{C}$ & 1.14178 & 0.47312 & -1.03612 \\
\hline $\mathrm{C}$ & 2.088 & 1.51573 & -1.33887 \\
\hline $\mathrm{C}$ & 3.29113 & 1.12523 & -1.90197 \\
\hline $\mathrm{C}$ & 3.7132 & -0.21735 & -1.87503 \\
\hline $\mathrm{C}$ & 2.96635 & -1.20128 & -1.24819 \\
\hline $\mathrm{C}$ & 3.60799 & -2.46281 & -0.70666 \\
\hline $\mathrm{C}$ & 2.03374 & 2.92104 & -0.75472 \\
\hline $\mathrm{C}$ & 4.38113 & -2.24303 & 0.68482 \\
\hline $\mathrm{C}$ & 2.316 & 2.95725 & 0.81978 \\
\hline $\mathrm{C}$ & 2.86096 & -0.62099 & 1.8875 \\
\hline $\mathrm{C}$ & 2.34717 & 0.67579 & 1.89606 \\
\hline $\mathrm{C}$ & 3.06288 & 1.7428 & 1.33422 \\
\hline $\mathrm{C}$ & 4.41433 & 1.51576 & 1.03299 \\
\hline $\mathrm{C}$ & 4.92436 & 0.21568 & 1.00847 \\
\hline $\mathrm{C}$ & 4.10121 & -0.88515 & 1.29313 \\
\hline $\mathrm{C}$ & -0.27289 & 0.69704 & -0.86914 \\
\hline $\mathrm{H}$ & 4.01329 & 1.88413 & -2.1896 \\
\hline $\mathrm{H}$ & 4.72535 & -0.44961 & -2.19864 \\
\hline $\mathrm{H}$ & 4.32836 & -2.85282 & -1.43331 \\
\hline $\mathrm{H}$ & 2.84422 & -3.22923 & -0.56318 \\
\hline $\mathrm{H}$ & 2.81058 & 3.5094 & -1.25244 \\
\hline $\mathrm{H}$ & 1.09294 & 3.4479 & -0.92511 \\
\hline $\mathrm{H}$ & 4.08289 & -3.05033 & 1.36267 \\
\hline $\mathrm{H}$ & 5.45499 & -2.35588 & 0.5016 \\
\hline $\mathrm{H}$ & 1.35361 & 3.02878 & 1.33838 \\
\hline $\mathrm{H}$ & 2.85717 & 3.8891 & 1.02172 \\
\hline $\mathrm{H}$ & 2.21166 & -1.43978 & 2.18308 \\
\hline $\mathrm{H}$ & 1.31688 & 0.82369 & 2.2107 \\
\hline $\mathrm{H}$ & 5.03418 & 2.33589 & 0.67577 \\
\hline $\mathrm{H}$ & 5.92984 & 0.04871 & 0.62696 \\
\hline $\mathrm{C}$ & -0.92894 & 1.95712 & -1.37631 \\
\hline $\mathrm{H}$ & -1.07038 & 2.67978 & -0.56476 \\
\hline $\mathrm{H}$ & -0.32696 & 2.42065 & -2.15615 \\
\hline $\mathrm{H}$ & -1.91666 & 1.73402 & -1.78532 \\
\hline $\mathrm{O}$ & 0.83631 & -1.84724 & -0.48979 \\
\hline $\mathrm{C}$ & -2.45155 & -0.10897 & -0.21097 \\
\hline $\mathrm{C}$ & -2.96212 & 0.60891 & 0.87608 \\
\hline $\mathrm{C}$ & -3.31295 & -0.74728 & -1.11126 \\
\hline
\end{tabular}




$\begin{array}{llcc}\mathrm{C} & -4.33812 & 0.71864 & 1.05137 \\ \mathrm{H} & -2.28266 & 1.07043 & 1.58414 \\ \mathrm{C} & -4.6894 & -0.64014 & -0.94568 \\ \mathrm{H} & -2.90208 & -1.32059 & -1.93409 \\ \mathrm{C} & -5.18107 & 0.09444 & 0.13344 \\ \mathrm{H} & -4.75571 & 1.27081 & 1.8835 \\ \mathrm{H} & -5.37585 & -1.1174 & -1.63323 \\ \mathrm{~B} & -0.41475 & -1.60246 & 0.21766 \\ \mathrm{~F} & -0.21305 & -1.43068 & 1.58789 \\ \mathrm{~F} & -1.29116 & -2.64168 & -0.03161 \\ \mathrm{~N} & -1.03066 & -0.25882 & -0.35639 \\ \mathrm{~N} & -6.63257 & 0.21035 & 0.31037 \\ \mathrm{O} & -7.36226 & -0.35826 & -0.50376 \\ \mathrm{O} & -7.04862 & 0.87208 & 1.26319\end{array}$

Coordinates of $\left(R_{p}\right) /\left(S_{p}\right)-3 e$ in the $\mathrm{S}_{1}$ state

\begin{tabular}{|c|c|c|c|}
\hline $\mathrm{C}$ & 1.591308 & -0.940207 & -0.815659 \\
\hline $\mathrm{C}$ & 1.071698 & 0.396281 & -0.993937 \\
\hline $\mathrm{C}$ & 2.009553 & 1.392406 & -1.288311 \\
\hline $\mathrm{C}$ & 3.255061 & 0.962796 & -1.867903 \\
\hline $\mathrm{C}$ & 3.700553 & -0.359733 & -1.835465 \\
\hline $\mathrm{C}$ & 2.977278 & -1.291939 & -1.117333 \\
\hline $\mathrm{C}$ & 3.644961 & -2.513944 & -0.532332 \\
\hline $\mathrm{C}$ & 1.969754 & 2.846473 & -0.839397 \\
\hline $\mathrm{C}$ & 4.572664 & -2.153557 & 0.723195 \\
\hline $\mathrm{C}$ & 2.400616 & 3.032959 & 0.683810 \\
\hline $\mathrm{C}$ & 3.189054 & -0.419065 & 1.949703 \\
\hline $\mathrm{C}$ & 2.652323 & 0.876763 & 1.944785 \\
\hline $\mathrm{C}$ & 3.244495 & 1.878477 & 1.183234 \\
\hline $\mathrm{C}$ & 4.550041 & 1.632317 & 0.694883 \\
\hline $\mathrm{C}$ & 5.102643 & 0.338382 & 0.725952 \\
\hline $\mathrm{C}$ & 4.349722 & -0.732099 & 1.205394 \\
\hline $\mathrm{C}$ & -0.386574 & 0.610297 & -0.864237 \\
\hline $\mathrm{H}$ & 3.920807 & 1.720622 & -2.266188 \\
\hline $\mathrm{H}$ & 4.695730 & -0.593338 & -2.194606 \\
\hline $\mathrm{H}$ & 4.281223 & -2.961226 & -1.299767 \\
\hline $\mathrm{H}$ & 2.895250 & -3.254656 & -0.252462 \\
\hline $\mathrm{H}$ & 2.692412 & 3.392215 & -1.450959 \\
\hline $\mathrm{H}$ & 1.008251 & 3.327502 & -1.001687 \\
\hline $\mathrm{H}$ & 4.369251 & -2.876838 & 1.517258 \\
\hline $\mathrm{H}$ & 5.617042 & -2.281320 & 0.429190 \\
\hline $\mathrm{H}$ & 1.507984 & 3.130633 & 1.307055 \\
\hline $\mathrm{H}$ & 2.938584 & 3.983409 & 0.741921 \\
\hline $\mathrm{H}$ & 2.624223 & -1.210097 & 2.429487 \\
\hline $\mathrm{H}$ & 1.689901 & 1.041810 & 2.417007 \\
\hline
\end{tabular}




\begin{tabular}{lrrc}
$\mathrm{H}$ & 5.096070 & 2.427100 & 0.193912 \\
$\mathrm{H}$ & 6.050544 & 0.155804 & 0.228959 \\
$\mathrm{C}$ & -1.052356 & 1.814418 & -1.465211 \\
$\mathrm{H}$ & -1.229326 & 2.590537 & -0.713060 \\
$\mathrm{H}$ & -0.458957 & 2.234119 & -2.277662 \\
$\mathrm{H}$ & -2.030314 & 1.518954 & -1.849643 \\
$\mathrm{O}$ & 0.833542 & -1.888795 & -0.372790 \\
$\mathrm{C}$ & -2.551158 & -0.165580 & -0.178963 \\
$\mathrm{C}$ & -3.057086 & 0.723588 & 0.783849 \\
$\mathrm{C}$ & -3.434416 & -0.932826 & -0.958574 \\
$\mathrm{C}$ & -4.425813 & 0.869153 & 0.951194 \\
$\mathrm{H}$ & -2.369241 & 1.291592 & 1.405178 \\
$\mathrm{C}$ & -4.803815 & -0.783111 & -0.806113 \\
$\mathrm{H}$ & -3.037677 & -1.632867 & -1.687468 \\
$\mathrm{C}$ & -5.323281 & 0.122710 & 0.150448 \\
$\mathrm{H}$ & -4.832086 & 1.544790 & 1.691218 \\
$\mathrm{H}$ & -5.499395 & -1.351824 & -1.407894 \\
$\mathrm{~B}$ & -0.483743 & -1.637429 & 0.289872 \\
$\mathrm{~F}$ & -0.257752 & -1.420683 & 1.641500 \\
$\mathrm{~F}$ & -1.279468 & -2.722651 & 0.047282 \\
$\mathrm{~N}$ & -1.115087 & -0.330681 & -0.326547 \\
$\mathrm{~N}$ & -6.701085 & 0.275705 & 0.303612 \\
$\mathrm{O}$ & -7.497335 & -0.429545 & -0.442474 \\
$\mathrm{O}$ & -7.142049 & 1.122755 & 1.184549 \\
& & & \\
\hline & & &
\end{tabular}

\section{References}

1. Frisch, M. J.; Trucks, G. W.; Schlegel, H. B.; Scuseria, G. E.; Robb, M. A.; Cheeseman, J. R.; Scalmani, G.; Barone, V.; Mennucci, B.; Petersson, G. A.; Nakatsuji, H.; Caricato, M.; Li, X.; Hratchian, H. P.; Izmaylov, A. F.; Bloino, J.; Zheng, G.; Sonnenberg, J. L.; Hada, M.; Ehara, M.; Toyota, K.; Fukuda, R.; Hasegawa, J.; Ishida, M.; Nakajima, T.; Honda, Y.; Kitao, O.; Nakai, H.; Vreven, T.; Montgomery, Jr., J. A.; Peralta, J. E.; Ogliaro, F.; Bearpark, M.; Heyd, J. J.; Brothers, E.; Kudin, K. N.; Staroverov, V. N.; Kobayashi, R.; Normand, J.; Raghavachari, K.; Rendell, A.; Burant, J. C.; Iyengar, S. S.; Tomasi, J.; Cossi, M.; Rega, N.; Millam, J. M.; Klene, M.; Knox, J. E.; Cross, J. B.; Bakken, V.; Adamo, C.; Jaramillo, J.; Gomperts, R.; Stratmann, R. E.; Yazyev, O.; Austin, A. J.; Cammi, R.; Pomelli, C.; Ochterski, J. W.; Martin, R. L.; Morokuma, K.; Zakrzewski, V. G.; Voth, G. A.; Salvador, P.; Dannenberg, J. J.; Dapprich, S.; Daniels, A. D.; Farkas, Ö.; Foresman, J. B.; Ortiz, J. V.; Cioslowski, J.; Fox, D. J. . Gaussian 09, revision d.01. Gaussian, Inc. (2009).

2. Zhang, Y. P.; Li, D. D.; Li, Y.; Yu, J. H. Solvatochromic AIE luminogens as supersensitive water detectors in organic solvents and highly efficient cyanide chemosensors in water, Chem. Sci., 2014, 5, 2710-2716.

3. Sheldrick, G. M., Crystal structure refinement with SHELXL. Acta Crystallogr C Struct Chem 2015, 71 (Pt 1), 3-8. 